\title{
Total Synthesis of (+)-Suaveolindole: Establishment of its Absolute Configuration
}

\author{
Emile J. Velthuisen and Samuel J. Danishefsky*
}

Laboratory for Bioorganic Chemistry, Sloan-Kettering Institute for Cancer

Research, 1275 York Ave, New York, New York, 10021, and

Department of Chemistry, Columbia University, Havemeyer Hall, 3000 Broadway, New York, New York 10027 


\section{General Information:}

General Experimental Details: Reactions involving air or moisture-sensitive reagents or intermediates were performed under argon or nitrogen atmosphere in glassware which had been oven dried, or flame-dried under high vacuum. Indicated reaction temperatures refer to those of the reaction bath, while room temperature (rt) is noted as $23{ }^{\circ} \mathrm{C}$. Preparative reactions were stirred magnetically. Tetrahydrofuran (THF), diethyl ether $\left(\mathrm{Et}_{2} \mathrm{O}\right)$, methylene chloride $\left(\mathrm{CH}_{2} \mathrm{Cl}_{2}\right)$, and toluene were obtained from a dry solvent system (activated alumina columns, positive pressure of argon). All other solvents were used as received in Sure/Seal bottles (Aldrich). Triethylamine $\left(\mathrm{Et}_{3} \mathrm{~N}\right)$, diisopropylethylamine $\left(i-\mathrm{Pr}_{2} \mathrm{NEt}\right)$, pyridine, 2,6-lutidine, and chlorotrimethylsilane (TMSCl) were distilled from $\mathrm{CaH}_{2}$ immediately prior to use. All other reagents were purchased from Aldrich at the highest commercial quality and used without further purification, with the exception of $\mathrm{Pd}\left(\mathrm{PPh}_{3}\right)_{4}$, and $\mathrm{Pd}_{2} \mathrm{dba}_{3}$, which were purchased from Strem and $\mathrm{PhNTf}_{2}$ which was purchased from Oakwood Chemical.

Instrumentation: Optical rotations were measured on a JASCO DIP-370 digital polarimeter at rt. Concentration (c) in $\mathrm{g} / 100 \mathrm{ml}$ and solvent are given in parentheses. Infrared spectra were obtained on a Perkin-Elmer 1600 FT-IR spectrophotometer neat or as a film in $\mathrm{CHCl}_{3}\left(\mathrm{NaCl}\right.$ plates). Absorption bands are noted in $\mathrm{cm}^{-1} .{ }^{1} \mathrm{H}-$ and ${ }^{13} \mathrm{C} \mathrm{NMR}$ spectra were recorded on a Bruker AMX-400, a Bruker DRX-500, or a Bruker AVII+600 spectrometer in $\mathrm{CDCl}_{3}$. Chemical shifts ( $\delta$-values) are reported in ppm with residual undeuterated $\mathrm{CHCl}_{3}$ as the internal standard (referenced to $7.26 \mathrm{ppm}$ for $1 \mathrm{H}-\mathrm{NMR}$ and $77.2 \mathrm{ppm}$ for 13C-NMR). Coupling constants $(\mathrm{J})(\mathrm{H}, \mathrm{H})$ are given in $\mathrm{Hz}$, spectral splitting patterns are designated as singlet $(\mathrm{s})$, doublet $(\mathrm{d})$, triplet $(\mathrm{t})$, quadruplet $(\mathrm{q})$, quintet (qunit), multiplet or more overlapping signals (m), apparent (app), broad signal (br). Low resolution mass spectra (ionspray, a variation of electrospray) were acquired on a PerkinElmer Sciex API 100 spectrometer. Samples were introduced by direct infusion. High resolution mass spectra data was collected at the CUNY Mass Spectrometry Facility at Hunter College on an Agilent Technologies 6210 high resolution time-of-flight mass spectrometer attached to an Agilent Technologies 1200 HPLC system. The sample was ionized by simultaneous electrospray and atmospheric pressure chemical ionization with data being collected for both positive and negative ionization using Agilent's Multimode source. Flash chromatography (FC) was performed with E. Merck silica gel (60, particle size $0.040-0.063 \mathrm{~mm}$ ) with loading approximately $50: 1(\mathrm{wt} / \mathrm{wt})$ silica gel/crude residue. Preparative thin layer chromatography (TLC) was performed with Whatman Partisil Plates $(20$ x $20 \mathrm{~cm}, 60 \AA, 200 \mu \mathrm{m})$. 
Scheme 1. Synthesis of Heck precursor 8.

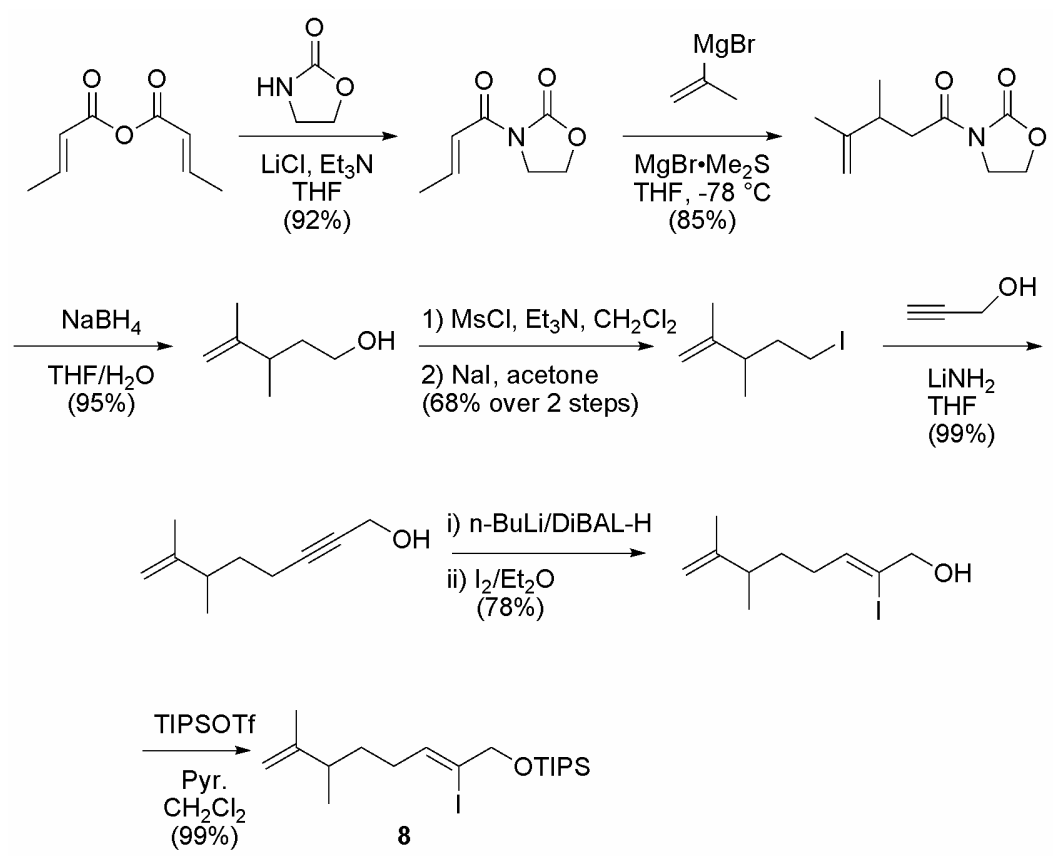

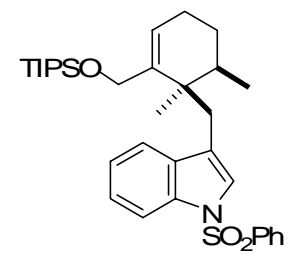

Chemical Formula: $\mathrm{C}_{33} \mathrm{H}_{4} \mathrm{NO}_{3} \mathrm{SS}$ Exact Mass: 565.30459 Molecular Weight: 565.88168
Indole 10. A solution of alkenyl iodide $8(0.5 \mathrm{~g}, 1.15 \mathrm{mmol})$, indole stannane 9 (0.94 g, $1.7 \mathrm{mmol})$, and $\mathrm{Bu}_{4} \mathrm{NCl}(0.35 \mathrm{~g}, 1.3$ mmol) in DMF (4 mL) was degassed with Ar. After $15 \mathrm{~min}$, $\mathrm{Ph}_{3}$ As (35 mg, $0.12 \mathrm{mmol}$ ) and $\mathrm{Pd}_{2} \mathrm{dba}_{3}$ (26 mg, $0.029 \mathrm{mmol}$ ) were added in one portion and the reaction was immediately submersed in a $105{ }^{\circ} \mathrm{C}$ oil bath. After stirring for $3 \mathrm{~h}$, the reaction was cooled to $23{ }^{\circ} \mathrm{C}$ and poured into $\mathrm{H}_{2} \mathrm{O}(10 \mathrm{~mL})$ and $\mathrm{Et}_{2} \mathrm{O}(10 \mathrm{~mL})$. The layers were partitioned, and the aqueous phase was extracted with $\mathrm{Et}_{2} \mathrm{O}(2 \times 20 \mathrm{~mL})$. The combined organic extracts were washed with $\mathrm{H}_{2} \mathrm{O}(5 \times 10 \mathrm{~mL})$, brine $(10 \mathrm{~mL})$, dried $\left(\mathrm{MgSO}_{4}\right)$, filtered and concentrated. The crude residue was purified by flash column chromatography on silica gel (1\% ethyl acetate - hexanes) to yield $0.36 \mathrm{~g}(55 \%)$ of $\mathbf{1 0}$ as a $4: 1$ mixture of diastereomers; major isomer: ${ }^{1} \mathrm{H} \mathrm{NMR}\left(\mathrm{CDCl}_{3}, 400 \mathrm{MHz}\right): \delta 7.99(\mathrm{~d}, J=8.1 \mathrm{~Hz}, 1 \mathrm{H}), 7.84(\mathrm{~d}, J=7.6 \mathrm{~Hz}, 2 \mathrm{H})$, 7.54-7.49 (m, 2H), 7.42-7.39 (m, 3H), 7.29-7.19 (m, 2H), 5.84 (bs, 1H), 4.10 (d, J= 13 $\mathrm{Hz}, 1 \mathrm{H}), 4.00(\mathrm{~d}, J=13 \mathrm{~Hz}, 1 \mathrm{H}), 2.87(\mathrm{~d}, J=14.5 \mathrm{~Hz}, 1 \mathrm{H}), 2.71(\mathrm{~d}, J=14.5 \mathrm{~Hz}, 1 \mathrm{H})$, 2.00-1.94 (m, 2H), 1.64-1.56 (m, 2H), 1.46-1.41 (m, 1H), 1.29-1.21 (m, 2H), $1.21(\mathrm{~s}$, $3 \mathrm{H}), 1.06-0.89(\mathrm{~m}, 33 \mathrm{H}) ;{ }^{13} \mathrm{C} \mathrm{NMR}\left(\mathrm{CDCl}_{3}, 125 \mathrm{MHz}\right): \delta 141.59,138.47,134.79$, $133.56,132.28,129.10,126.64,124.45,124.39$, 123.53, 122.98, 120.84, 120.13, 113.57, 64.48, 40.12, 38.24, 29.76, 26.29, 25.67, 23.88, 18.09, 17.71, 16.15, 12.29, 11.96; HRMS $\left(\mathrm{ESI},[\mathrm{M}+\mathrm{Na}]^{+}\right.$) calculated for: $\mathrm{C}_{33} \mathrm{H}_{47} \mathrm{NO}_{3} \mathrm{SSiNa}$ 588.2938. Found: 588.2933. 


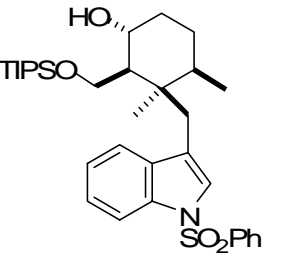

Chemical Formula: $\mathrm{C}_{33} \mathrm{H}_{49} \mathrm{NO}_{4} \mathrm{SS}$ Exact Mass: 583.31516 Molecular Weight: 583.89696

Alcohol 11. A solution of $\mathrm{BH}_{3} \cdot \mathrm{THF}(0.53 \mathrm{~mL}, 0.53 \mathrm{mmol}, 1.0$ $\mathrm{M}$ THF) was added dropwise to a solution of $\mathbf{1 0}(0.1 \mathrm{~g}, 0.18$ $\mathrm{mmol})$ in THF $(0.5 \mathrm{~mL})$ at $0{ }^{\circ} \mathrm{C}$. The mixture was stirred for 3 $\mathrm{h}$ allowing it to warm to $23{ }^{\circ} \mathrm{C}$. Excess borane was quenched by the slow addition of $\mathrm{H}_{2} \mathrm{O}(36 \mu \mathrm{L})$, and the oraganoborane was sequentially treated with $\mathrm{NaBO}_{3} \bullet 4 \mathrm{H}_{2} \mathrm{O}(0.14 \mathrm{~g}, 0.9 \mathrm{mmol})$ and $\mathrm{H}_{2} \mathrm{O}(0.18 \mathrm{~mL})$. The reaction mixture was stirred for $16 \mathrm{~h}$ at $23{ }^{\circ} \mathrm{C}$, whereupon it was diluted with $\mathrm{Et}_{2} \mathrm{O}(5 \mathrm{~mL})$ and $\mathrm{H}_{2} \mathrm{O}$ $(5 \mathrm{~mL})$. The layers were partitioned and the aqueous phase was extracted with $\mathrm{Et}_{2} \mathrm{O}(2 \times 5 \mathrm{~mL})$, the combined organic extracts were washed with brine $(10 \mathrm{~mL})$, dried $\left(\mathrm{MgSO}_{4}\right)$, filtered and concentrated. The crude residue was purified by flash column chromatography on silica gel ( $10 \%$ ethyl acetate - hexanes) to yield $56 \mathrm{mg}$ (56\%) of 11 as a colorless oil: ${ }^{1} \mathrm{H}$ NMR $\left(\mathrm{CDCl}_{3}, 400 \mathrm{MHz}\right): \delta 7.99(\mathrm{~d}, J=7.8 \mathrm{~Hz}, 1 \mathrm{H})$, $7.88(\mathrm{~d}, J=7.8 \mathrm{~Hz}, 2 \mathrm{H}), 7.53-7.52(\mathrm{~m}, 2 \mathrm{H}), 7.45-7.42(\mathrm{~m}, 2 \mathrm{H}), 7.33-7.26(\mathrm{~m}, 3 \mathrm{H})$, 4.14-4.06 (m, 1H), $3.70(\mathrm{~d}, J=9.7 \mathrm{~Hz}, 1 \mathrm{H}), 3.52(\mathrm{dd}, J=14.6,9.7 \mathrm{~Hz}, 1 \mathrm{H}), 2.65(\mathrm{~d}, J=$ $22 \mathrm{~Hz}, 1 \mathrm{H}), 2.52(\mathrm{~d}, J=22 \mathrm{~Hz}, 1 \mathrm{H}), 1.46-1.34(\mathrm{~m}, 3 \mathrm{H}), 1.22-1.19(\mathrm{~m}, 3 \mathrm{H}), 0.90-0.83$ $(\mathrm{m}, 28 \mathrm{H}) ;{ }^{13} \mathrm{C} \mathrm{NMR}\left(\mathrm{CDCl}_{3}, 125 \mathrm{MHz}\right): \delta 138.36134 .73,133.69,131.93,129.21,126.71$, $124.65,124.62,123.37,120.31,119.85,113.71,73.18,70.57,65.77,56.49,43.96,40.43$, $35.58,28.71,25.09,23.37,17.70,17.64,16.46,11.31$. HRMS (ESI, [M+Na] $\left.]^{+}\right)$calculated for: $\mathrm{C}_{33} \mathrm{H}_{49} \mathrm{NO}_{4} \mathrm{SSiNa}$ 606.3043. Found: 606.3042 .

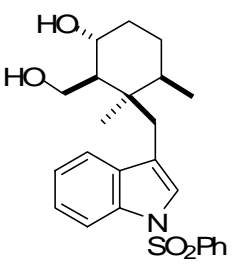

Chemical Formula: $\mathrm{C}_{24} \mathrm{H}_{29} \mathrm{NO}_{4} \mathrm{~S}$ Exact Mass: 427.18173 Molecular Weight: 427.55636

Deprotection of 11: A solution of $11(10 \mathrm{mg}, 0.017 \mathrm{mmol})$ in THF $(100 \mu \mathrm{L})$ and $\mathrm{AcOH}(4 \mu \mathrm{L}, 0.068 \mathrm{mmol})$ at $23{ }^{\circ} \mathrm{C}$ was treated with TBAF $(34 \mu \mathrm{L}, 0.034 \mathrm{mmol}, 1.0 \mathrm{M} \mathrm{THF})$. After stirring for $16 \mathrm{~h}$, the reaction was concentrated in vacuo and the crude residue was purified by preparative TLC ( $20 \%$ ethyl acetate - hexanes) to afford the title compound as a white foam (7.0 mg, 100\% yield): ${ }^{1} \mathrm{H} \mathrm{NMR}\left(\mathrm{CDCl}_{3}, 500 \mathrm{MHz}\right): \delta 7.95$ (d, $J$ $=8.2 \mathrm{~Hz}, 1 \mathrm{H}), 7.79(\mathrm{~d}, J=7.9 \mathrm{~Hz}, 2 \mathrm{H}), 7.47-7.42(\mathrm{~m}, 2 \mathrm{H}), 7.36$ (dd, $J=7.9,7.8 \mathrm{~Hz}, 2 \mathrm{H}), 7.25-7.24(\mathrm{~m}, 1 \mathrm{H}), 7.21-7.18(\mathrm{~m} \mathrm{2H})$, 3.97 (dt, $J=10.75,4.75 \mathrm{~Hz}, 1 \mathrm{H}), 3.44-3.40(\mathrm{~m}, 1 \mathrm{H}), 3.30(\mathrm{dd}, J=10.85,8.3 \mathrm{~Hz}, 1 \mathrm{H})$, 2.64 (bs, $1 \mathrm{H}), 2.50(\mathrm{~d}, J=15 \mathrm{~Hz}, 1 \mathrm{H}), 2.45(\mathrm{~d}, J=15 \mathrm{~Hz}, 1 \mathrm{H}), 2.02-1.98(\mathrm{~m}, 1 \mathrm{H}), 1.52-$ $1.31(\mathrm{~m}, 5 \mathrm{H}), 1.25-1.17(\mathrm{~m}, 3 \mathrm{H}), 1.15(\mathrm{~s}, 3 \mathrm{H}), 0.78(\mathrm{~d}, J=6.9 \mathrm{~Hz}, 3 \mathrm{H}) ;{ }^{13} \mathrm{C}$ NMR $\left(\mathrm{CDCl}_{3}, 125 \mathrm{MHz}\right): \delta 138.21,134.95,133.76,132.16,129.23,128.34,126.62,124.97$, $124.69,123.34,120.77,119.85,113.93,73.55,63.95,57.91,43.85,41.06,36.42,29.69$, 28.97, 24.95, 23.57, 16.49. HRMS (ESI, $[\mathrm{M}+\mathrm{Na}]^{+}$) calculated for: $\mathrm{C}_{24} \mathrm{H}_{29} \mathrm{NO}_{4} \mathrm{SNa}$ 450.1709. Found: 450.1707. 
Structural Determination of Deprotected 11:

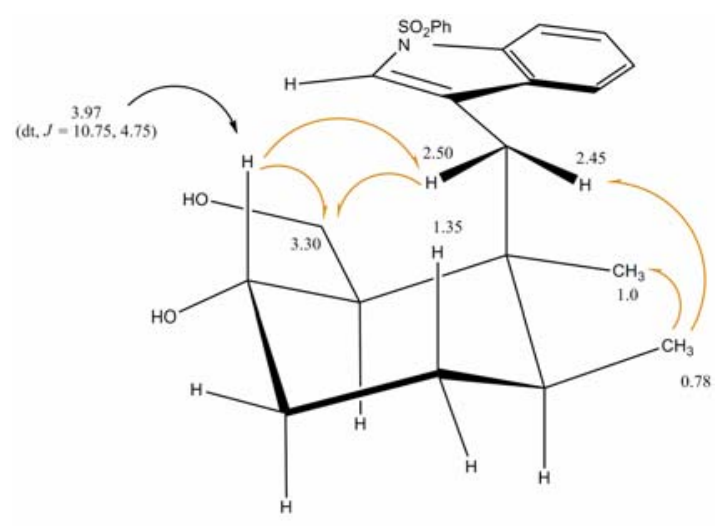

Chemical Formula: $\mathrm{C}_{11} \mathrm{H}_{16} \mathrm{O}$ Exact Mass: 164.1201

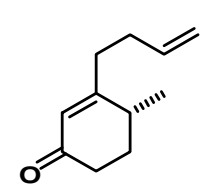
Molecular Weight: 164.2441

\section{(R)-3-(but-3-enyl)-4-methylcyclohex-2-enone (13). ${ }^{1}$}

A solution of 4-bromo-1-butene $(1.0 \mathrm{~mL}, 10 \mathrm{mmol})$ in THF $(4.6 \mathrm{~mL})$ was added dropwise over a $20 \mathrm{~min}$ period to a stirring suspension of $\mathrm{Mg}$ turnings (240 mg, $10 \mathrm{mmol})$ in THF $(2.0 \mathrm{~mL})$. Following completion of the addition, the reaction mixture was stirred for $30 \mathrm{~min}$ at $23{ }^{\circ} \mathrm{C}$ and cannulated into a solution of $(R)-\mathbf{1 2}^{2}$ in THF $(17 \mathrm{~mL})$ at $-78{ }^{\circ} \mathrm{C}$. After $20 \mathrm{~min}$, the reaction was warmed to $23{ }^{\circ} \mathrm{C}$ and maintained at this temperature for $3 \mathrm{~h}$. The reaction was quenched by cooling to $0^{\circ} \mathrm{C}$ and cautiously adding $\mathrm{H}_{2} \mathrm{O}(10 \mathrm{~mL})$. The layers were partitioned and the aqueous phase was extracted with $\mathrm{Et}_{2} \mathrm{O}(2 \times 20 \mathrm{~mL})$, the combined organic extracts were washed with brine $(2 \times 20 \mathrm{~mL})$, dried $\left(\mathrm{MgSO}_{4}\right)$, filtered and concentrated. The crude carbinol was then dissolved in $\mathrm{CH}_{2} \mathrm{Cl}_{2}(6 \mathrm{~mL})$ and added to a flask charged with PCC (3.9 g, $18.2 \mathrm{mmol})$ and $\mathrm{CH}_{2} \mathrm{Cl}_{2}(18 \mathrm{~mL})$ at $23{ }^{\circ} \mathrm{C}$. The resulting reaction mixture was maintained at this temperature for $16 \mathrm{~h}$, decanted, and the remaining polymeric material was thoroughly washed with $\mathrm{Et}_{2} \mathrm{O}(3 \times 30 \mathrm{~mL})$. The organic layers were combined, and sequentially washed with aqueous $\mathrm{NaOH}(2 \times 20 \mathrm{~mL}, 5 \%)$, aqueous $\mathrm{HCl}(2 \times 20 \mathrm{~mL}$, $5 \%)$, saturated aqueous $\mathrm{NaHCO}_{3}(2 \times 20 \mathrm{~mL})$ and brine $(2 \times 20 \mathrm{~mL})$. The layers were dried $\left(\mathrm{MgSO}_{4}\right)$, filtered, and concentrated. The crude residue was purified by flash column chromatography on silica gel $(5 \rightarrow 10 \%$ ethyl acetate - hexanes $)$ to yield $0.7 \mathrm{~g}$ $(47 \%)$ of $(R)-13$ as a pale yellow oil: $[\alpha]^{22}=+15^{\circ}\left(\mathrm{c}=2.3, \mathrm{CHCl}_{3}\right) ;{ }^{1} \mathrm{H} \mathrm{NMR}\left(\mathrm{CDCl}_{3}\right.$, $500 \mathrm{MHz}): \delta 5.82-5.75(\mathrm{~m}, 2 \mathrm{H}), 5.06-4.98(\mathrm{~m}, 2 \mathrm{H}), 2.51-2.41(\mathrm{~m}, 2 \mathrm{H}), 2.34-2.19(\mathrm{~m}$, $5 \mathrm{H}), 2.13-2.06(\mathrm{~m}, 1 \mathrm{H}), 1.80-1.74(\mathrm{~m}, 1 \mathrm{H}), 1.19(\mathrm{~d}, J=7.6 \mathrm{~Hz}, 3 \mathrm{H}) ;{ }^{13} \mathrm{C} \mathrm{NMR}\left(\mathrm{CDCl}_{3}\right.$, $125 \mathrm{MHz}): \delta 199.88,169.53,137.28,125.42,115.79,34.99,34.42,33.32,31.40,30.37$, 18.0; HRMS (ESI, $[\mathrm{M}+\mathrm{H}]^{+}$) calculated for: $\mathrm{C}_{11} \mathrm{H}_{17} \mathrm{O}$ 165.1273. Found: 165.1272.

(1) Access to racemic 13 was accomplished by a Stork-Danheiser reaction of 3-ethoxy-6-methylcyclohex2-enone and but-3-enylmagnesium bromide in 94\% yield, see: Stork, G.; Danheiser, R. L. J. Org. Chem. 1973, 38, 1775-1776.

(2) (a) Organic Syntheses; Wiley \& Sons: New York, 2005; Vol. 82, p 108. (b) Schreiber, S. L. J. Am. Chem. Soc. 1980, 102, 6165-6166. 


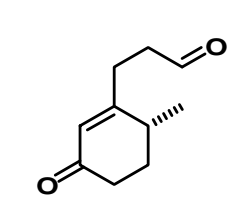

Chemical Formula: $\mathrm{G}_{10} \mathrm{H}_{14} \mathrm{O}_{2}$ Exact Mass: 166.10 Molecular Weight: 166.22

(R)-3-(6-methyl-3-oxocyclohex-1-enyl)propanal (14). A suspension of $13(0.5 \mathrm{~g}, 3.0 \mathrm{mmol})$ and $\mathrm{NaHCO}_{3}(12 \mathrm{mg}, 0.15$ $\mathrm{mmol})$ in $\mathrm{MeOH}(30 \mathrm{~mL})$ at $-78{ }^{\circ} \mathrm{C}$ was treated with ozone until TLC indicated the reaction was complete. The reaction mixture was purged with argon for $10 \mathrm{~min}$, and a solution of DMS $(1.1 \mathrm{ml}, 15$ mmol $)$ in $\mathrm{CH}_{2} \mathrm{Cl}_{2}(1.1 \mathrm{~mL})$ was added. The reaction was maintained at $23{ }^{\circ} \mathrm{C}$ for $16 \mathrm{~h}$, filtered through a pad of celite and concentrated. The crude residue $(0.44 \mathrm{~g}, 88 \%)$ was dried in vacuo (450 mtorr) for $1 \mathrm{~h}$ and used without further purification: $[\alpha]^{22}=+48^{\circ}\left(\mathrm{c}=1.3, \mathrm{CHCl}_{3}\right) .{ }^{1} \mathrm{H} \mathrm{NMR}\left(\mathrm{CDCl}_{3}, 500 \mathrm{MHz}\right)$ : $\delta 9.82(\mathrm{~s}, 1 \mathrm{H}), 5.72(\mathrm{~s}, 1 \mathrm{H}), 2.71-2.67(\mathrm{~m}, 2 \mathrm{H}), 2.63-2.43(\mathrm{~m}, 4 \mathrm{H}), 2.35-2.29(\mathrm{~m}, 1 \mathrm{H})$, 2.15-2.08 (m, 1H), 1.82-1.76 (m, 1H), $1.21(\mathrm{~d}, J=6.9 \mathrm{~Hz}, 3 \mathrm{H}) ;{ }^{13} \mathrm{C}$ NMR $\left(\mathrm{CDCl}_{3}, 125\right.$ $\mathrm{MHz}): \delta 200.21,199.26,167.72,124.87,41.0,34.10,33.49,30.13,27.29,17.83$; HRMS (ESI, $[\mathrm{M}+\mathrm{H}]^{+}$) calculated for: $\mathrm{C}_{10} \mathrm{H}_{15} \mathrm{O}_{2}$ 167.1066. Found 167.1063.

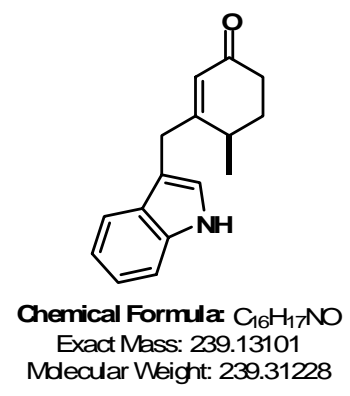

(R)-3-((1H-indol-3-yl)methyl)-4-methylcyclohex-2-enone. A mixture of 2-iodoaniline (6.4 g, $29.2 \mathrm{mmol}),(R)-14(4.4 \mathrm{~g}, 26.5$ $\mathrm{mmol})$, and DABCO (8.9 g, $79.5 \mathrm{mmol})$ in DMF $(130 \mathrm{~mL})$ was degassed with Ar for $20 \mathrm{~min} . \mathrm{Pd}(\mathrm{OAc})_{2}(300 \mathrm{mg}, 1.33 \mathrm{mmol})$ was added to the reaction, and the resulting reaction mixture was heated to $85^{\circ} \mathrm{C}$ for $16 \mathrm{~h}$. The reaction was cooled to $23{ }^{\circ} \mathrm{C}$ and was diluted with $\mathrm{H}_{2} \mathrm{O}(100 \mathrm{~mL})$. The aqueous phase was extracted with $\mathrm{Et}_{2} \mathrm{O}(3 \times 50 \mathrm{~mL})$, and the combined organic phases were washed with brine $(50 \mathrm{~mL})$, dried $\left(\mathrm{MgSO}_{4}\right)$, filtered and concentrated. The crude residue was purified by flash column chromatography on silica gel $(30 \%$ ethyl acetate - hexanes) to yield $4.3 \mathrm{~g}(68 \%)$ of the title compound as a yellow foam: $[\alpha]^{22}{ }_{\mathrm{D}}=$ $+81^{\circ}\left(\mathrm{c}=0.9, \mathrm{CHCl}_{3}\right) .{ }^{1} \mathrm{H} \mathrm{NMR}\left(\mathrm{CDCl}_{3}, 500 \mathrm{MHz}\right): \delta 8.03(\mathrm{bs}, 1 \mathrm{H}), 7.47(\mathrm{~d}, J=8.6 \mathrm{~Hz}$, $1 \mathrm{H}), 7.27(\mathrm{~d}, J=9.5 \mathrm{~Hz}, 1 \mathrm{H}), 7.19(\mathrm{dd}, J=15.3,7.6 \mathrm{~Hz}, 1 \mathrm{H}), 7.09$ (dd, $J=16.2,7.6 \mathrm{~Hz}$, $1 \mathrm{H}), 7.03(\mathrm{~s}, 1 \mathrm{H}), 5.84(\mathrm{~s}, 1 \mathrm{H}), 3.68(\mathrm{~s}, 3 \mathrm{H}), 2.54-2.46(\mathrm{~m}, 2 \mathrm{H}), 2.31$ (ddd, $J=11.5,11.5$,

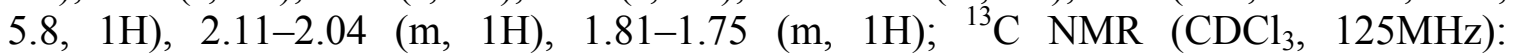
$\delta 200.28,169.56,136.69,127.41,125.78,122.95,122.16,119.59,118.71,111.33,111.16$, 34.09, 32.57, 31.97, 30.22, 17.83; HRMS (ESI, $[\mathrm{M}+\mathrm{H}]^{+}$) calculated for: $\mathrm{C}_{16} \mathrm{H}_{18} \mathrm{NO}$ 240.1382. Found: 240.1383.

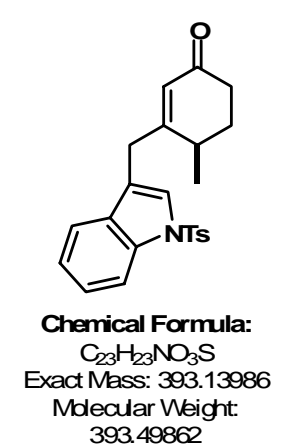

(R)-4-methyl-3-((1-tosyl-1H-indol-3-yl)methyl)cyclohex-2-enone (15). Tosyl chloride $(0.87 \mathrm{~g}, 4.60 \mathrm{mmol})$ was added to a mixture of (R)-3-((1H-indol-3-yl)methyl)-4-methylcyclohex-2-enone $\quad(1.0 \mathrm{~g}$, $4.18 \mathrm{mmol})$, and TBAB $(0.14 \mathrm{~g}, 0.42 \mathrm{mmol})$ in $\mathrm{NaOH}(14 \mathrm{~mL}, 15 \%$ aq), and benzene $(13 \mathrm{~mL})$ at $23{ }^{\circ} \mathrm{C}$. The reaction was maintained at this temperature for $2 \mathrm{~h}$, and monitored by ${ }^{1} \mathrm{H}$ NMR analysis. Upon consumption of starting material the layers were partitioned, and the aqueous phase was extracted with $\mathrm{Et}_{2} \mathrm{O}(3 \times 20 \mathrm{~mL})$. The combined organic phases were washed with brine $(50 \mathrm{~mL})$, dried $\left(\mathrm{MgSO}_{4}\right)$, filtered and concentrated. The crude residue was purified by flash column chromatography on silica gel $(20 \rightarrow 30 \%$ ethyl acetate - hexanes $)$ to yield $1.5 \mathrm{~g}$ 
(91\%) of 15 as a yellow foam: $[\alpha]^{22}{ }_{\mathrm{D}}=+48^{\circ}\left(\mathrm{c}=2.5, \mathrm{CHCl}_{3}\right) .{ }^{1} \mathrm{H}$ NMR $\left(\mathrm{CDCl}_{3}, 500\right.$ MHz): $\delta 7.98(\mathrm{~d}, J=8.0 \mathrm{~Hz}, 1 \mathrm{H}), 7.74(\mathrm{~d}, J=8.8 \mathrm{~Hz}, 1 \mathrm{H}), 7.38(\mathrm{~s}, 1 \mathrm{H}), 7.36-7.21(\mathrm{~m}$, 2H), 7.23-7.19 (m, 3H), $5.70(\mathrm{~s}, 1 \mathrm{H}), 3.59(\mathrm{~s}, 1 \mathrm{H}), 2.53-2.44(\mathrm{~m}, 2 \mathrm{H}), 2.35-2.29(\mathrm{~m}$, $4 \mathrm{H}), 2.11-2.05(\mathrm{~m}, 1 \mathrm{H}), 1.82-1.76(\mathrm{~m}, 1 \mathrm{H}), 1.25(\mathrm{~d}, J=7.2 \mathrm{~Hz}, 3 \mathrm{H}) ;{ }^{13} \mathrm{C} \mathrm{NMR}\left(\mathrm{CDCl}_{3}\right.$, 125MHz): $\delta$ 199.39, 166.72, 145.08, 135.4, 135.06, 130.48, 125.01, 124.54, 123.38, 119.4 $8,118.13,113.91,34.03,31.63,30.12,21.60,17.81$; HRMS (ESI, $\left.[\mathrm{M}+\mathrm{H}]^{+}\right)$calculated for: $\mathrm{C}_{23} \mathrm{H}_{24} \mathrm{NO}_{3} \mathrm{~S}$ 394.1471. Found: 394.1474 .

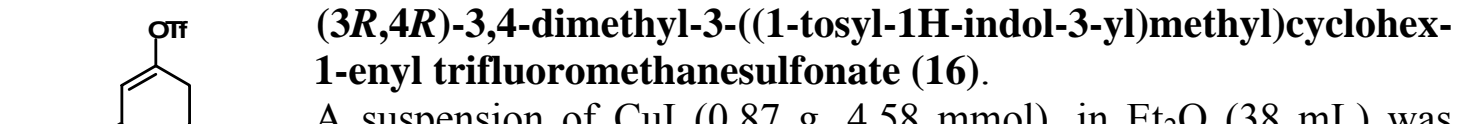

A suspension of $\mathrm{CuI}(0.87 \mathrm{~g}, 4.58 \mathrm{mmol})$, in $\mathrm{Et}_{2} \mathrm{O}(38 \mathrm{~mL})$ was cooled to $-30{ }^{\circ} \mathrm{C}$ and treated dropwise with a solution of $\mathrm{MeLi}(7.4$ $\mathrm{mL}, 9.17 \mathrm{mmol}, 1.25 \mathrm{M}$ in $\mathrm{Et}_{2} \mathrm{O}$ ). After $10 \mathrm{~min}$, the reaction mixture was warmed to $0{ }^{\circ} \mathrm{C}$ resulting in a colorless, homogeneous solution. A solution of enone $15(1.5 \mathrm{~g}, 3.82 \mathrm{mmol})$ in $\mathrm{Et}_{2} \mathrm{O}-\mathrm{THF}$ $(6 \mathrm{~mL}-1.5 \mathrm{~mL})$ was added over a $20 \mathrm{~min}$ period, resulting in an orange precipitate. After stirring for an additional $30 \mathrm{~min}$, the Molecular: 541 as treated with a solution of $\mathrm{PhNTf}_{2}(3.14 \mathrm{~g}, 8.79 \mathrm{mmol})$ in THF (9 $\mathrm{mL}$ ). After $20 \mathrm{~min}$, saturated aqueous $\mathrm{NH}_{4} \mathrm{Cl}$ was added, and the layers partitioned. The aqueous phase was extracted with $\mathrm{Et}_{2} \mathrm{O}(2 \times 20 \mathrm{~mL})$, and the combined organic extracts were washed with brine $(2 \times 20 \mathrm{~mL})$, dried $\left(\mathrm{MgSO}_{4}\right)$, filtered and concentrated. The residue was purified by flash column chromatography on silica gel $(5 \rightarrow 10 \%$ ethyl acetate - hexanes) to yield $(R, R)-\mathbf{1 6}$ as a 9:1 mixture of diastereomers and an inseparable mixture ${ }^{3}$ with PhNHTf: ${ }^{1} \mathrm{H}$ NMR $\left(\mathrm{CDCl}_{3}, 600 \mathrm{MHz}\right): \delta 7.92(\mathrm{~d}, J=8.4 \mathrm{~Hz}, 1 \mathrm{H}), 7.68(\mathrm{~d}$, $J=8.4 \mathrm{~Hz}, 2 \mathrm{H}), 7.32(\mathrm{~d}, J=7.9 \mathrm{~Hz}, 1 \mathrm{H}), 7.22(\mathrm{dd}, J=7.6,7.4 \mathrm{~Hz}, 1 \mathrm{H}), 7.17-7.12(\mathrm{~m}$, $3 \mathrm{H}), 2.60(\mathrm{~d}, J=13.8 \mathrm{~Hz}, 1 \mathrm{H}), 2.54(\mathrm{~d}, J=13.8 \mathrm{~Hz}, 1 \mathrm{H}), 2.36-2.27(\mathrm{~m}, 2 \mathrm{H}), 2.25(\mathrm{~s}$, $3 \mathrm{H}), 1.72-1.67(\mathrm{~m}, 2 \mathrm{H}), 1.63-1.55(\mathrm{~m}, 1 \mathrm{H}), 1.00(\mathrm{~d}, J=7.0 \mathrm{~Hz}, 3 \mathrm{H}), 0.95(\mathrm{~s}, 3 \mathrm{H}) ;{ }^{13} \mathrm{C}$ NMR

$\left(\mathrm{CDCl}_{3}\right.$,

$150 \mathrm{MHz})$ :

$\delta 148.19,144.91,135.16,135.06,131.86,128.84,127.10,126.74,125.06,124.60,123.18$ , 120.06, 118.43, 113.73, 39.01, 38.07, 30.67, 30.67, 27.35, 27.09, 26.11, 21.51, 15.22; HRMS (ESI, [M+Na] $]^{+}$) calculated for: $\mathrm{C}_{25} \mathrm{H}_{26} \mathrm{NO}_{5} \mathrm{~F}_{3} \mathrm{~S}_{2} \mathrm{Na}$ 564.1096. Found: 564.1096.

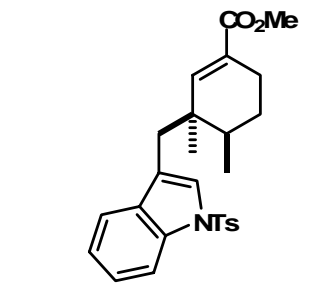

Chemical Formula: $\mathrm{C}_{26} \mathrm{H}_{29} \mathrm{NO}_{4} \mathrm{~S}$ Exact Mass: 451.18 Molecular Weight: 45158
$(3 R, 4 R)$-Methyl yl)methyl)cyclohex-1-enecarboxylate. A mixture of enol triflate 16 (2.0 g, $3.8 \mathrm{mmol}), i-\operatorname{Pr}_{2} \mathrm{EtN}$ (3.3 mL, $\left.19 \mathrm{mmol}\right), \mathrm{MeOH}$ (13 $\mathrm{mL}, 0.3 \mathrm{M})$, and DMF $(20 \mathrm{~mL})$ was purged with Ar for $5 \mathrm{~min}$. $\mathrm{Pd}\left(\mathrm{PPh}_{3}\right)_{4}(0.22 \mathrm{~g}, 0.19 \mathrm{mmol})$ was added and $\mathrm{CO}$ was bubbled through the reaction mixture for $30 \mathrm{~min}$. $\mathrm{Et}_{2} \mathrm{O}(20 \mathrm{~mL})$ and $\mathrm{H}_{2} \mathrm{O}$ $(20 \mathrm{~mL})$ were added, and the layers partitioned. The aqueous phase was extracted with $\mathrm{Et}_{2} \mathrm{O}(2 \times 20 \mathrm{~mL})$, and the combined organic extracts were washed with water $(5 \times 20 \mathrm{~mL})$, brine $(2 \times$ $20 \mathrm{~mL})$, dried $\left(\mathrm{MgSO}_{4}\right)$, filtered and concentrated. The residue was purified by flash column chromatography on silica gel $(5 \rightarrow 10 \%$ ethyl acetate - hexanes $)$ to yield the title

\footnotetext{
${ }^{3}$ Note, that on small scale, recrystallization of $\mathbf{1 8}$ from $5 \%$ ethyl acetate was successful in removing majority of the PhNHTf.
} 
compound $\left(0.76 \mathrm{~g}, 45 \%\right.$ from 15) as a white foam: $[\alpha]^{22}{ }_{\mathrm{D}}=+40^{\circ}\left(\mathrm{c}=1.1, \mathrm{CHCl}_{3}\right) .{ }^{1} \mathrm{H}$ NMR $\left(\mathrm{CDCl}_{3}, 600 \mathrm{MHz}\right): \delta 7.90(\mathrm{~d}, J=8.3 \mathrm{~Hz}, 1 \mathrm{H}), 7.69(\mathrm{~d}, J=8.3 \mathrm{~Hz}, 2 \mathrm{H}), 7.30(\mathrm{~d}, J$ $=7.9 \mathrm{~Hz}, 1 \mathrm{H}), 7.22-7.18(\mathrm{~m}, 1 \mathrm{H}), 7.14(\mathrm{~d}, J=8.2 \mathrm{~Hz}, 2 \mathrm{H}), 7.11-7.10(\mathrm{~m}, 1 \mathrm{H}), 6.40(\mathrm{~s}$, $1 \mathrm{H}), 2.60(\mathrm{dd}, J=22.3,13.8,2 \mathrm{H}), 2.38-2.35(\mathrm{~m}, 1 \mathrm{H}), 2.26(\mathrm{~s}, 3 \mathrm{H}), 2.18-2.12(\mathrm{~m}, 1 \mathrm{H})$, $1.67-1.61(\mathrm{~m}, 1 \mathrm{H}), 1.58-1.51(\mathrm{~m}, 2 \mathrm{H}), 1.00(\mathrm{~d}, J=7.6 \mathrm{~Hz}, 3 \mathrm{H}), 0.91(\mathrm{~s}, 3 \mathrm{H}) ;{ }^{13} \mathrm{C}$ NMR $\left(\mathrm{CDCl}_{3}\right.$,

$150 \mathrm{MHz})$ :

$\delta 167.97,147.84,144.74,135.17,135.05,132.16,129.80,129.12,126.81,125.06,124.42$ , 122.98, 120.35, 119.19, 113.69, 51.62, 38.46, 38.17, 30.76, 26.75, 25.57, 24.27, 21.56, 1 6.0; HRMS (ESI, $[\mathrm{M}+\mathrm{H}]^{+}$) calculated for: $\mathrm{C}_{26} \mathrm{H}_{30} \mathrm{NO}_{4} \mathrm{~S} 452.1890$. Found: 452.1887 .

2-((3R,4R)-3,4-dimethyl-3-((1-tosyl-1H-indol-3-

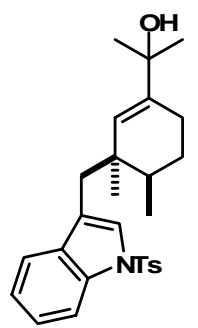

Chemical Formula: $\mathrm{C}_{27} \mathrm{H}_{33} \mathrm{NO}_{3} \mathrm{~S}$

Exact Mass: 451.22 Molecular Weight: 451.62 yl)methyl)cyclohex-1-enyl)propan-2-ol. A solution the above
$\alpha, \beta$-unsaturated ester $(0.76 \mathrm{~g}, 1.69 \mathrm{mmol})$ in THF $(13 \mathrm{~mL})$ at $0{ }^{\circ} \mathrm{C}$ was treated with $\mathrm{MeLi}\left(4.0 \mathrm{~mL}, 5.0 \mathrm{mmol}, 1.25 \mathrm{M}\right.$ in $\left.\mathrm{Et}_{2} \mathrm{O}\right)$. After $10 \mathrm{~min}$, the reaction was poured into half-saturated $\mathrm{NH}_{4} \mathrm{Cl}(20$ $\mathrm{mL})$ and $\mathrm{Et}_{2} \mathrm{O}(20 \mathrm{~mL})$ and the layers partitioned. The aqueous phase was extracted with $\mathrm{Et}_{2} \mathrm{O}(2 \times 10 \mathrm{~mL})$, and the combined organic extracts were washed with brine $(2 \times 10 \mathrm{~mL})$, dried $\left(\mathrm{MgSO}_{4}\right)$, filtered, and concentrated. The residue was purified by flash column chromatography on silica gel $(10 \rightarrow 20 \%$ ethyl acetate - hexanes) to yield the title compound $(0.64 \mathrm{~g}, 84 \%)$ as a white foam: $[\alpha]^{22}{ }_{\mathrm{D}}=+$ $52^{\circ}\left(\mathrm{c}=1.1, \mathrm{CHCl}_{3}\right) .{ }^{1} \mathrm{H} \mathrm{NMR}\left(\mathrm{CDCl}_{3}, 500 \mathrm{MHz}\right): \delta 7.99(\mathrm{~d}, J=8.3 \mathrm{~Hz}, 1 \mathrm{H}), 7.75(\mathrm{~d}, J$ $=8.3 \mathrm{~Hz}, 1 \mathrm{H}), 7.37(\mathrm{~d}, J=7.9 \mathrm{~Hz}, 1 \mathrm{H}), 7.31(\mathrm{~s}, 1 \mathrm{H}), 7.29-7.26(\mathrm{~m}, 2 \mathrm{H}), 7.21-7.18(\mathrm{~m}$, $3 \mathrm{H}), 2.67(\mathrm{~d}, J=13.5 \mathrm{~Hz}, 1 \mathrm{H}), 2.52(\mathrm{~d}, J=13.5 \mathrm{~Hz}, 1 \mathrm{H}), 2.38(\mathrm{~s}, 3 \mathrm{H}), 2.21-2.18(\mathrm{~m}$, $1 \mathrm{H}), 2.06-2.00(\mathrm{~m}, 1 \mathrm{H}), 1.66-1.57(\mathrm{~m}, 3 \mathrm{H}), 1.15(\mathrm{~s}, 3 \mathrm{H}), 1.06-1.05(\mathrm{~m}, 6 \mathrm{H}), 1.00(\mathrm{~s}$, ${ }_{3 \mathrm{H}) ;}{ }^{13} \mathrm{C} \quad \mathrm{NMR} \quad\left(\mathrm{CDCl}_{3}, \quad 150 \mathrm{MHz}\right)$ : $\delta 144.92,142.04,135.44,135.33,132.70,130.19,129.97,128.66,127.62,126.95,124.85$ , 124.64, 122.84, 120.80, 120.49, 114.03,72.77, 44.85, 39.04, 37.48, 31.33, 28.86, 28.78, 28.04, 27.94, 26.86, 24.65, 21.76, 16.10; HRMS (ESI, $\left.[\mathrm{M}+\mathrm{Na}]^{\dagger}\right)$ calculated for: $\mathrm{C}_{27} \mathrm{H}_{33} \mathrm{NO}_{3} \mathrm{SNa}$ 474.2073. Found: 474.2069.

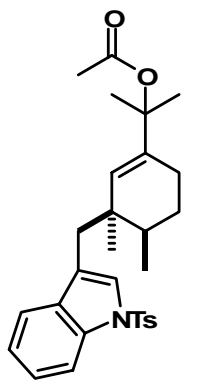

Chemical Formula: $\mathrm{C}_{29} \mathrm{H}_{35} \mathrm{NO}_{4} \mathrm{~S}$ Exact Mass: 493.23 Molecular Weight: 493.66

2-((3R,4R)-3,4-dimethyl-3-((1-tosyl-1H-indol-3yl)methyl)cyclohex-1-enyl)propan-2-yl acetate (17). A mixture of the above tertiary allylic alcohol $(0.5 \mathrm{~g}, 1.11 \mathrm{mmol}), i-\mathrm{Pr}_{2} \mathrm{EtNH}$ (2.0 mL, $11.1 \mathrm{mmol}), \mathrm{Ac}_{2} \mathrm{O}$ (0.63 mL, $\left.6.65 \mathrm{mmol}\right)$, and DMAP (13 $\mathrm{mg}, 0.11 \mathrm{mmol})$ in $\mathrm{CH}_{2} \mathrm{Cl}_{2}(2 \mathrm{ml})$ was maintained at $23{ }^{\circ} \mathrm{C}$ for 16 h. $\mathrm{Et}_{2} \mathrm{O}(10 \mathrm{~mL})$ and $\mathrm{H}_{2} \mathrm{O}(10 \mathrm{~mL})$ were added, and the layers partitioned. The aqueous phase was extracted with $\mathrm{Et}_{2} \mathrm{O}(2 \times 10$ $\mathrm{mL}$ ), and the combined organic extracts were washed with brine $(20 \mathrm{~mL})$, dried $\left(\mathrm{MgSO}_{4}\right)$, filtered, and concentrated. The residue was purified by flash column chromatography on silica gel $(5 \%$ ethyl acetate - hexanes) to yield $\mathbf{1 7}(0.45 \mathrm{~g}, 83 \%)$ as a white foam: $[\alpha]^{22}{ }_{\mathrm{D}}=+67^{\circ}\left(\mathrm{c}=1.1, \mathrm{CHCl}_{3}\right) .{ }^{1} \mathrm{H} \mathrm{NMR}\left(\mathrm{CDCl}_{3}, 500 \mathrm{MHz}\right): \delta 7.86(\mathrm{~d}, J=8.3 \mathrm{~Hz}, 1 \mathrm{H})$, 7.64-7.62 (d, $J=8.3 \mathrm{~Hz}, 2 \mathrm{H}), 7.35(\mathrm{~d}, J=7.9 \mathrm{~Hz}, 1 \mathrm{H}), 7.17-7.14(\mathrm{~m}, 2 \mathrm{H}), 7.08-7.06(\mathrm{~m}$, $3 \mathrm{H}), 2.53(\mathrm{~d}, J=13.5 \mathrm{~Hz}, 1 \mathrm{H}), 2.42(\mathrm{~d}, J=13.5 \mathrm{~Hz}, 1 \mathrm{H}), 2.21(\mathrm{~s}, 3 \mathrm{H}), 1.95-1.92(\mathrm{~m}$, $1 \mathrm{H}), 1.82(\mathrm{~s}, 3 \mathrm{H}), 1.52-1.46(\mathrm{~m}, 4 \mathrm{H}), 1.23(\mathrm{~s}, 3 \mathrm{H}), 1.18-1.13(\mathrm{~m}, 4 \mathrm{H}), 0.93(\mathrm{~d}, J=5.9$ 
$\mathrm{Hz}, \quad 3 \mathrm{H}), \quad 0.85 \quad(\mathrm{~s}, \quad 3 \mathrm{H}) ; \quad{ }^{13} \mathrm{C} \quad \mathrm{NMR} \quad\left(\mathrm{CDCl}_{3}, \quad 150 \mathrm{MHz}\right)$ $\delta 169.76,144.62,138.77,135.36,135.02,132.49,129.71,126.74,124.97,124.25,122.78$ , 120.75, 120.02, 113.52, 38.74, 37.39, 30.82, 27.44, 26.72, 26.52, 25.74, 24.03, 22.67, 22 $.13,21.53,15.90$; HRMS (ESI, $[\mathrm{M}+\mathrm{Na}]^{+}$) calculated for: $\mathrm{C}_{29} \mathrm{H}_{35} \mathrm{NO}_{4} \mathrm{SNa} 516.2179$. Found: 516.2174 .

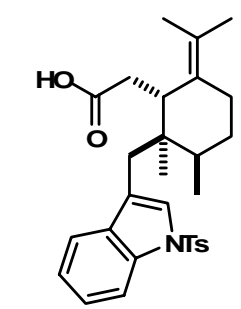

Chemical Formula: $\mathrm{C}_{29} \mathrm{H}_{55} \mathrm{NO}_{4} \mathrm{~S}$ Exact Mass: 493.23 Molecular Weight: 493.66

2-((1S,2R,3R)-2,3-dimethyl-6-(propan-2-ylidene)-2-((1-tosyl1H-indol-3-yl)methyl)cyclohexyl)acetic acid (18). A solution of 17 (0.45 g, $0.91 \mathrm{mmol})$ in THF $(4.0 \mathrm{~mL})$ was added dropwise over a 5 min period to a mixture of LiHMDS $(3.0 \mathrm{~mL}, 3.0 \mathrm{mmol}, 1.0$ $\mathrm{M}$ THF $)$ and TMSCl $(0.35 \mathrm{~mL}, 2.74 \mathrm{mmol})$ in THF $(5.0 \mathrm{~mL})$ at $78{ }^{\circ} \mathrm{C}$. The reaction mixture was warmed to $23{ }^{\circ} \mathrm{C}$ over a $2 \mathrm{~h}$ period, and maintained at this temperature for $16 \mathrm{~h} . \mathrm{Et}_{2} \mathrm{O}(10 \mathrm{~mL})$ and $2 \mathrm{M} \mathrm{HCl}(10 \mathrm{~mL})$ were added, and the layers partitioned. The aqueous phase was extracted with $\mathrm{Et}_{2} \mathrm{O}(3 \times 5 \mathrm{~mL})$, and the combined organic extracts were washed with brine $(10 \mathrm{~mL})$, dried $\left(\mathrm{MgSO}_{4}\right)$, filtered, and concentrated. The crude residue was purified by flash column chromatography on silica gel $(10 \%$ ethyl acetate - hexanes with $1 \% \mathrm{AcOH})$ to yield $0.25 \mathrm{~g}(56 \%)$ of the title compound as a white foam: $[\alpha]^{22}{ }_{\mathrm{D}}=+89^{\circ}\left(\mathrm{c}=0.9, \mathrm{CHCl}_{3}\right) .{ }^{1} \mathrm{H}$ NMR $\left(\mathrm{CDCl}_{3}, 600 \mathrm{MHz}\right): \delta 8.06(\mathrm{~d}, J=8.3 \mathrm{~Hz}, 1 \mathrm{H}), 7.80(\mathrm{~d}, J=8.3 \mathrm{~Hz}, 2 \mathrm{H}), 7.49-7.45$ $(\mathrm{m}, 2 \mathrm{H}), 7.39-7.34(\mathrm{~m}, 1 \mathrm{H}), 7.28-7.24(\mathrm{~m}, 3 \mathrm{H}), 3.26-3.20(\mathrm{~m}, 1 \mathrm{H}), 2.75(\mathrm{~d}, J=14.5 \mathrm{~Hz}$, $1 \mathrm{H}), 2.72-2.69(\mathrm{~m}, 1 \mathrm{H}), 2.59(\mathrm{~d}, J=14.5 \mathrm{~Hz}, 1 \mathrm{H}), 2.52-2.46(\mathrm{~m}, 2 \mathrm{H}), 2.36(\mathrm{~s}, 3 \mathrm{H}), 2.16-$ $2.04(\mathrm{~m}, 1 \mathrm{H}), 1.85-1.75(\mathrm{~m}, 1 \mathrm{H}), 1.73(\mathrm{~s}, 3 \mathrm{H}), 1.65-1.63(\mathrm{~m}, 1 \mathrm{H}), 1.53-1.45(\mathrm{~m}, 1 \mathrm{H})$, $1.32(\mathrm{~s}, 3 \mathrm{H}), 1.04(\mathrm{~d}, J=6.7 \mathrm{~Hz}, 1 \mathrm{H}), 0.95(\mathrm{~s}, 3 \mathrm{H}) ;{ }^{13} \mathrm{C} \mathrm{NMR}\left(\mathrm{CDCl}_{3}\right.$, $150 \mathrm{MHz}): \delta 182.06,146.76,137.13,137.11,135.03,131.89,131.84,128.74,128.02,126$. $63,126.41,124.90,122.38,122.03,115.83,43.97,42.50,38.43,36.14,32.80,28.24,27.0$ 8, 26.71, 23.54, 22.82, 22.30, 17.89; HRMS (ESI, [M+H] $]^{+}$calculated for: $\mathrm{C}_{29} \mathrm{H}_{36} \mathrm{NO}_{4} \mathrm{~S}$ 494.2360. Found: 494.2365 .

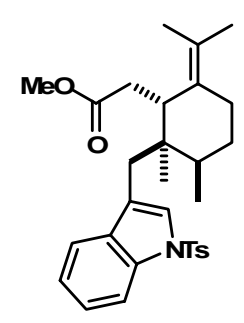

Methyl 2,3-dimethyl-6-(propan-2-ylidene)-2-((1-tosyl-1Hindol-3-yl)methyl)cyclohexyl)acetate. A solution of $18(5.0 \mathrm{mg}$, $0.01 \mathrm{mmol})$ in $\mathrm{MeOH}(0.5 \mathrm{~mL})$ was treated with $\mathrm{H}_{2} \mathrm{SO}_{4}(15 \mu \mathrm{L}$, catalytic) at $23{ }^{\circ} \mathrm{C}$ and maintained for $16 \mathrm{~h}$. The reaction mixture was poured into $10 \%$ aq. $\mathrm{NaOH}(1 \mathrm{~mL})$ and extracted with $\mathrm{Et}_{2} \mathrm{O}$ $(3 \times 2 \mathrm{~mL})$. The combined organic extracts were washed with brine $(5 \mathrm{~mL})$, dried $\left(\mathrm{MgSO}_{4}\right)$, filtered and concentrated. The crude residue was purified by preparative TLC $(20 \%$ ethyl acetate hexanes) to afford the title compound as a colorless oil $(5 \mathrm{mg}$, $100 \%$ yield): ${ }^{1} \mathrm{H}$ NMR $\left(\mathrm{CDCl}_{3}, 600 \mathrm{MHz}\right): \delta 7.89(\mathrm{~d}, J=8.3 \mathrm{~Hz}$, $1 \mathrm{H}), 7.69(\mathrm{~d}, J=8.3 \mathrm{~Hz}, 2 \mathrm{H}), 7.34-7.32(\mathrm{~m}, 2 \mathrm{H}), 7.14-1.11(\mathrm{~m}, 3 \mathrm{H}), 3.49(\mathrm{~s}, 3 \mathrm{H}), 3.08$ (dd, $J=9.5,5.9 \mathrm{~Hz}, 1 \mathrm{H}), 2.58-2.53$ (m, 3H), 2.44 (d, $J=14.6 \mathrm{~Hz}, 1 \mathrm{H}), 2.35-2.29$ (m, 2 H), 2.25 (s, $3 \mathrm{H}), 1.94-1.89(\mathrm{~m}, 1 \mathrm{H}), 1.71-1.66(\mathrm{~m}, 1 \mathrm{H}), 1.57$ (s, $3 \mathrm{H}), 1.49-1.47$ (m, 4 $\mathrm{H}), 1.36-1.27(\mathrm{~m}, 4 \mathrm{H}), 6.80(\mathrm{~d}, J=6.8 \mathrm{~Hz}, 3 \mathrm{H}), 0.74(\mathrm{~s}, 3 \mathrm{H}) ;{ }^{13} \mathrm{C} \mathrm{NMR}\left(\mathrm{CDCl}_{3}\right.$, $150 \mathrm{MHz})$ :

$\delta 173.72,144.55,135.17,135.02,132.99,130.17,129.77,126.76,125.53,124.65,124.28$ , 122.82, 120.37, 119.99, 113.74, 51.27, 42.05, 40.38, 36.32, 34.14, 30.77, 26.17, 25.09, 2 
4.38, 21.53, 20.71, 20.26, 15.83; LRMS (ESI, $[\mathrm{M}+\mathrm{H}]^{+}$) calculated for: $\mathrm{C}_{30} \mathrm{H}_{38} \mathrm{NO}_{4} \mathrm{~S} 508$. Found: 508.

\section{Structural Determination for the Methyl Ester Derivative of 18:}

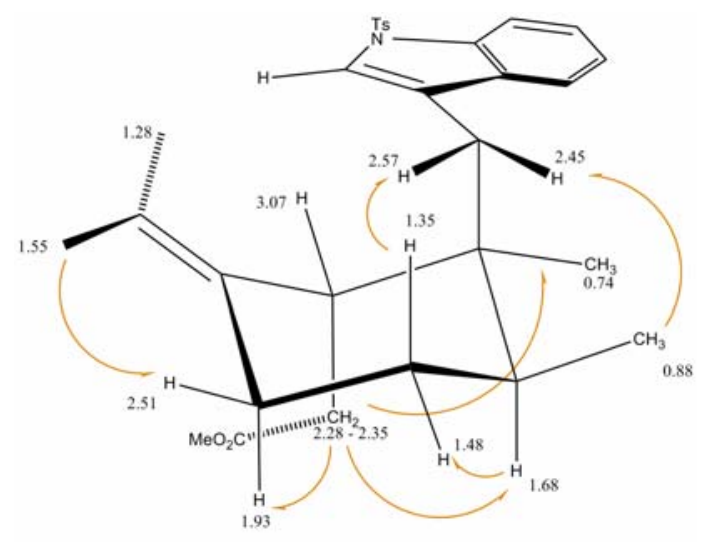

NOESY magor correlations

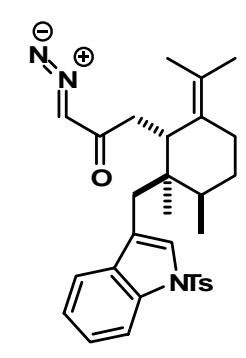

Chemical Formula: $\mathrm{C}_{30} \mathrm{H}_{35} \mathrm{~N}_{3} \mathrm{O}_{3} \mathrm{~S}$ Exact Mass: 517.23991 Molecular Weight: 517.68220
1-diazo-3-((1S,2R,3R)-2,3-dimethyl-6-(propan-2-ylidene)-2((1-tosyl-1H-indol-3-yl)methyl)cyclohexyl)propan-2-one. To a solution of carboxylic acid $18(108 \mathrm{mg}, 0.22 \mathrm{mmol})$ in $\mathrm{CH}_{2} \mathrm{Cl}_{2}(2$ $\mathrm{mL})$ at $0{ }^{\circ} \mathrm{C}$, was added oxalyl chloride $(0.55 \mathrm{~mL}, 1.1 \mathrm{mmol})$, followed by DMF $(25 \mu \mathrm{l})$. After stirring at $0{ }^{\circ} \mathrm{C}$ for $45 \mathrm{~min}$, all solvent was removed in vacuo. The crude acid chloride was dried in vacuo (450 mtorr) for $2 \mathrm{~h}$ and used without any further purification.

Note in the next step, diazomethane was dried with sodium metal for 15 min immediately prior to use. Diazomethane is toxic and explosive. Extreme caution should be exercised when preparing and handling diazomethane. All reactions should be carried out in a well-ventilated fume hood and behind a blast shield.

The crude acid chloride was dissolved in THF $(1 \mathrm{~mL})$, cooled to $0{ }^{\circ} \mathrm{C}$, and treated sequentially with $i-\operatorname{Pr}_{2} \mathrm{EtNH}(0.2 \mathrm{~mL}, 1.1 \mathrm{mmol})$ and diazomethane $(6.6 \mathrm{~mL}, 2.2 \mathrm{mmol})$. The reaction mixture was warmed to $23{ }^{\circ} \mathrm{C}$ and maintained at this temperature for 30 min. The reaction mixture was poured into saturated aqueous $\mathrm{NH}_{4} \mathrm{Cl}(5 \mathrm{~mL})$ and extracted with $\mathrm{Et}_{2} \mathrm{O}(3 \times 5 \mathrm{~mL})$. The combined organic extracts were washed with brine $(5 \mathrm{~mL})$, dried $\left(\mathrm{MgSO}_{4}\right)$, filtered, and concentrated. The crude residue was purified by flash column chromatography on silica gel $(2 \rightarrow 20 \%$ ethyl acetate - hexanes $)$ to yield the title compound as a white foam $(70 \mathrm{mg}, 62 \%)$ : ${ }^{1} \mathrm{H}$ NMR $\left(\mathrm{C}_{6} \mathrm{D}_{6}, 600 \mathrm{MHz}\right): \delta 8.54(\delta, J=$ $8.3 \mathrm{~Hz}, 1 \mathrm{H}), 8.21$ (d, $J=8.3 \mathrm{~Hz}, 2 \mathrm{H}), 8.03$ (s, 1H), 7.61-7.59 (d, J=1 Hz, 1H), 7.31 (dd, $J=7.4,7.4 \mathrm{~Hz}, 1 \mathrm{H}), 7.11(\mathrm{~d}, J=8.0 \mathrm{~Hz}, 2 \mathrm{H}), 4.42(\mathrm{~s}, 1 \mathrm{H}), 3.66-3.63(\mathrm{~m}, 1 \mathrm{H}), 2.81-2.79$ $(\mathrm{m}, 1 \mathrm{H}), 2.60(\mathrm{~d}, J=14.4 \mathrm{~Hz}, 1 \mathrm{H}), 2.33-2.30(\mathrm{~m}, 1 \mathrm{H}), 2.00(\mathrm{~s}, 3 \mathrm{H}), 1.79(\mathrm{~s}, 3 \mathrm{H}), 1.70(\mathrm{~s}$, $3 \mathrm{H}), 1.66-1.51(\mathrm{~m}, 3 \mathrm{H}), 1.07(\mathrm{~d}, J=6.4 \mathrm{~Hz}, 3 \mathrm{H}), 0.92(\mathrm{~s}, 3 \mathrm{H}) ;{ }^{13} \mathrm{C} \mathrm{NMR}\left(\mathrm{C}_{6} \mathrm{D}_{6}\right.$, 150MHz): $\delta 193.56,144.33,135.91,135.74,133.53,130.73,130.16,127.22,125.57,124$. $62,123.09,120.28,120.23,114.35,40.59,36.70,30.89,26.16,25.70,24.61,21.07,21.04$ 
, 20.21, 15.83, 5.61; HRMS (ESI, [M+H] $]^{+}$calculated for: $\mathrm{C}_{30} \mathrm{H}_{36} \mathrm{~N}_{3} \mathrm{O}_{3} \mathrm{~S}$ 518.2471. Found: 518.2475. IR (NaCl) $2096 \mathrm{~cm}^{-1}$.

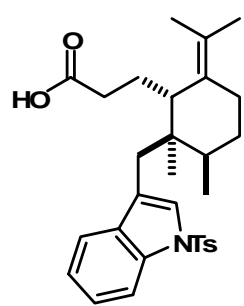

Chemical Fomula: $\mathrm{C}_{30} \mathrm{H}_{3} \mathrm{NO}_{4} \mathrm{~S}$

Exact Mass: 507.24

Molecular Weight: 507.68

3-((1S,2R,3R)-2,3-dimethyl-6-(propan-2-ylidene)-2-((1-tosyl1H-indol-3-yl)methyl)cyclohexyl)propanoic acid. The purified diazoketone from the preceding step is dissolved in THF $(0.54$ $\mathrm{mL})$ and $\mathrm{H}_{2} \mathrm{O}(0.05 \mathrm{~mL})$. The reaction mixture is cooled to $-25^{\circ} \mathrm{C}$ and protected from light. A solution of $\mathrm{CF}_{3} \mathrm{CO}_{2} \mathrm{Ag}(4.0 \mathrm{mg}, 0.017$ $\mathrm{mmol})$ in $\mathrm{Et}_{3} \mathrm{~N}(60 \mu \mathrm{L}, 0.41 \mathrm{mmol})$ was added in one portion and the reaction was warmed to $23{ }^{\circ} \mathrm{C}$ over a two hour period. After stirring for $16 \mathrm{~h}, 2 \mathrm{M} \mathrm{HCl}(1 \mathrm{~mL})$ was added and the aqueous phase was extracted with $\mathrm{Et}_{2} \mathrm{O}(3 \times 5 \mathrm{~mL})$. The combined organic extracts were washed with brine $(2 \mathrm{~mL})$, dried $\left(\mathrm{MgSO}_{4}\right)$, filtered, and concentrated. The crude residue was purified by flash column chromatography on silica gel $(5 \rightarrow 50 \%$ ethyl acetate - hexanes) to yield the title compound as a white foam $(50 \mathrm{mg}, 74 \%):[\alpha]^{22} \mathrm{D}=+$ $62^{\circ}\left(\mathrm{c}=1.1, \mathrm{CHCl}_{3}\right) .{ }^{1} \mathrm{H} \mathrm{NMR}\left(\mathrm{CDCl}_{3}, 600 \mathrm{MHz}\right): \delta 8.00(\mathrm{~d}, J=8.3 \mathrm{~Hz}, 1 \mathrm{H}), 7.72(\mathrm{~d}, J=$ $8.3 \mathrm{~Hz}, 2 \mathrm{H}), 7.38(\mathrm{~d}, J=7.7 \mathrm{~Hz}, 1 \mathrm{H}), 7.30-7.29(\mathrm{~m}, 2 \mathrm{H}), 7.22-7.18(\mathrm{~m}, 3 \mathrm{H}), 2.65$ (d, $J=$ $14.5 \mathrm{~Hz}, 1 \mathrm{H}), 2.58-2.55(\mathrm{~m}, 1 \mathrm{H}), 2.47(\mathrm{~d}, J=14.5 \mathrm{~Hz}, 1 \mathrm{H}), 2.42-2.39(\mathrm{~m}, 1 \mathrm{H}), 2.32$ (s, $3 \mathrm{H}), 2.18-1.94(\mathrm{~m}, 2 \mathrm{H}), 1.87-1.73(\mathrm{~m}, 3 \mathrm{H}), 1.71-1.65(\mathrm{~m}, 1 \mathrm{H}), 1.63(\mathrm{~s}, 3 \mathrm{H}), 1.55-1.53$ $(\mathrm{m}, 1 \mathrm{H}), 1.43-1.38(\mathrm{~m}, 1 \mathrm{H}), 1.06(\mathrm{~s}, 3 \mathrm{H}), 0.94(\mathrm{~d}, J=6.7 \mathrm{~Hz}, 3 \mathrm{H}) ;{ }^{13} \mathrm{C} \mathrm{NMR}\left(\mathrm{CDCl}_{3}\right.$, $150 \mathrm{MHz}): \delta 193.56^{4}, 144.72,135.24,135.11,133.15,130.08,129.66,125.75,124.36,124$ $.17,122.85,121.05,120.06,113.80 .44 .04,40.57,36.17,31.96,30.89,26.62,25.23,24.7$ $5,22.69,21.46,20.79,20.06,15.84$; HRMS $\quad\left(\mathrm{ESI}, \quad[\mathrm{M}+\mathrm{Na}]^{+}\right)$calculated for: $\mathrm{C}_{30} \mathrm{H}_{37} \mathrm{NO}_{4} \mathrm{SNa}$ 530.2335. Found: 530.2333. IR ( $\left.\mathrm{NaCl}\right) 1705 \mathrm{~cm}^{-1}$.

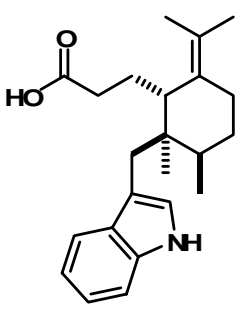

Chemical Fommula: $\mathrm{C}_{23} \mathrm{H}_{31} \mathrm{NO}_{2}$

Exact Mass: 353.24 Molecular Weight: 353.50
Suaveolindole (1). A 1.0 M stock solution of sodium naphthalenide was prepared by added $\mathrm{Na}(0.23 \mathrm{~g}, 10 \mathrm{mmol})$ to a solution of naphthalene $(1.3 \mathrm{~g}, 10 \mathrm{mmol})$ in DME $(10 \mathrm{~mL})$ at 23 ${ }^{\circ} \mathrm{C}$. After stirring for $90 \mathrm{~m}$, the freshly prepared sodium napthalenide $(1.0 \mathrm{~mL}, 1.0 \mathrm{mmol}, 1.0 \mathrm{M}$ in DME) was transferred to a solution of the tosyl protected indole $(52 \mathrm{mg}, 0.10 \mathrm{mmol})$ in DME $(1 \mathrm{~mL})$ at $23^{\circ} \mathrm{C}$. The reaction mixture was maintained at this temperature for $20 \mathrm{~min}$, poured into $2 \mathrm{M} \mathrm{HCl}(1 \mathrm{~mL})$ and extracted with $\mathrm{Et}_{2} \mathrm{O}(3 \times 2 \mathrm{~mL})$. The combined organic extracts were washed with brine $(5 \mathrm{~mL})$, dried $\left(\mathrm{MgSO}_{4}\right)$, filtered, and concentrated. The crude residue was purified by HPLC ( $2 \%$ IPA - hexanes; $4.6 \times 250 \mathrm{~mm}$ column, Silica $\left.5 \mu \mathrm{M} ; \mathrm{R}_{\mathrm{T}}=6.6 \mathrm{~min}\right)$ to afford suaveolindole $(1,33 \mathrm{mg}, 94 \%)$ as a white foam: $[\alpha]^{22}{ }_{\mathrm{D}}=+32^{\circ}(\mathrm{c}=1.0$, $\left.\mathrm{CD}_{3} \mathrm{OD}\right) .{ }^{1} \mathrm{H}$ NMR $\left(\mathrm{CD}_{3} \mathrm{OD}, 600 \mathrm{MHz}\right): 10.10$ (bs, $\left.0.4 \mathrm{H}\right), 7.43(\mathrm{~d}, J=7.9 \mathrm{~Hz}, 1 \mathrm{H}), 7.28$ $(\mathrm{d}, J=8.1 \mathrm{~Hz}, 1 \mathrm{H}), 7.02(\mathrm{dd}, J=7.7,7.3 \mathrm{~Hz}, 1 \mathrm{H}), 6.99(\mathrm{~s}, 1 \mathrm{H}), 6.94(\mathrm{dd}, J=7.9,7.1 \mathrm{~Hz}$, $1 \mathrm{H}), 2.72(\mathrm{~d}, J=14.5,1 \mathrm{H}), 2.68-2.61(\mathrm{~m}, 3 \mathrm{H}), 2.08-1.85(\mathrm{~m}, 4 \mathrm{H}), 1.80-1.72(\mathrm{~m}, 2 \mathrm{H})$, $1.70(\mathrm{~s}, 3 \mathrm{H}), 1.56-1.46(\mathrm{~m}, 2 \mathrm{H}), 1.35(\mathrm{~s}, 3 \mathrm{H}), 1.00(\mathrm{~m}, 6 \mathrm{H}) ;{ }^{13} \mathrm{C}$ NMR $\left(\mathrm{CD}_{3} \mathrm{OD}, 150\right.$ $\mathrm{MHz})$ :

$\delta 178.22,137.70,132.35,130.78,126.23,124.33,121.80,120.13,119.16,113.27,111.92$

${ }^{4}$ Visible when $\mathrm{LB}=10$ 
, 45.57, 41.86, 37.75, 33.11, 32.31, 27.89, 26.09, 25.91, 24.0, 21.67, 20.33, 16.39; HRMS (ESI, $[\mathrm{M}+\mathrm{H}]^{+}$) calculated for: $\mathrm{C}_{23} \mathrm{H}_{32} \mathrm{NO}_{2}$ 354.2427. Found: 354.2427 . 


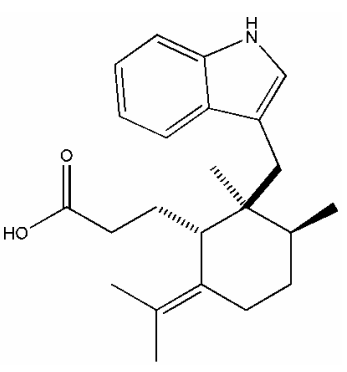

EJV-IV-91

(synthetic)

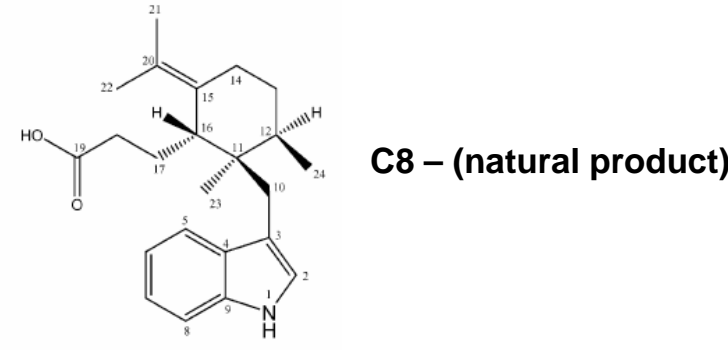

\section{C8 and C8 preaursors MC on Staphylococaus aureus ( Suave4)}

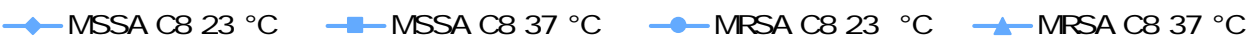

$\rightarrow$ MSSA IV- $9123^{\circ} \mathrm{C} \rightarrow$ - MSSA IV- $9137^{\circ} \mathrm{C} \rightarrow$ MRSA IV- $9123^{\circ} \mathrm{C} \rightarrow$ - MRSA IV- $9137^{\circ} \mathrm{C}$

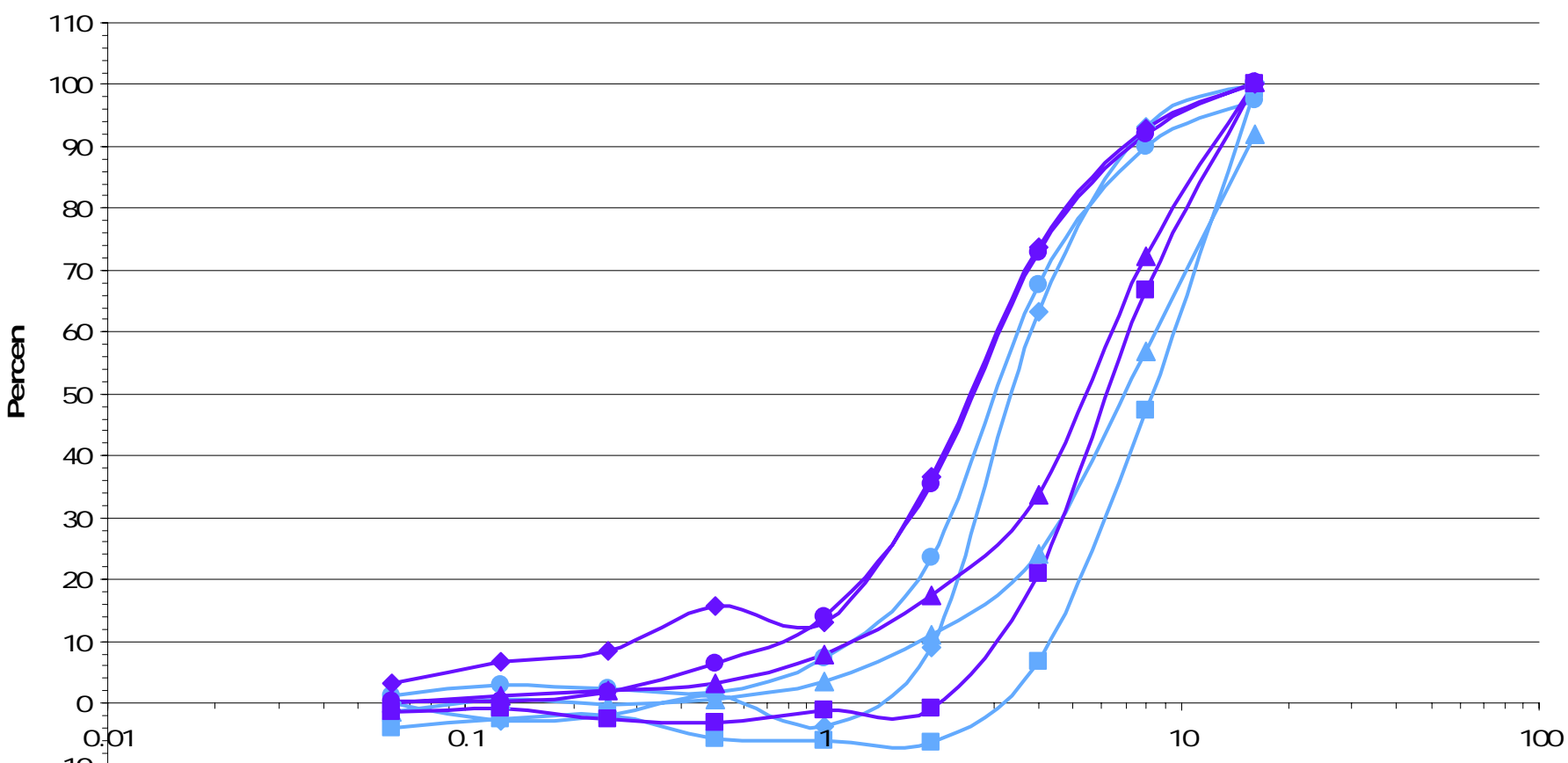

s Suake 4

-10
-20

Compound $[\mathrm{C}](\mu \mathrm{g} / \mathrm{ml})$ 


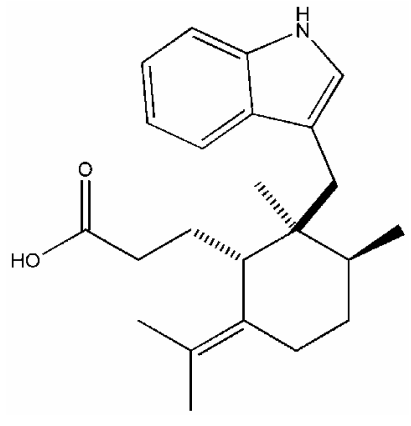

EJV-IV-91

(synthetic)
C8 - (natural product)

sSuake4

\section{C8 and C8 precursors MC on Staphylococaus epidermidis (Suave4)}

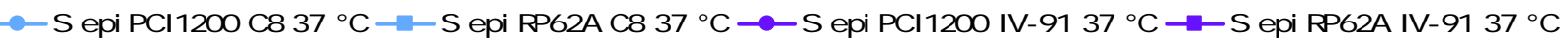

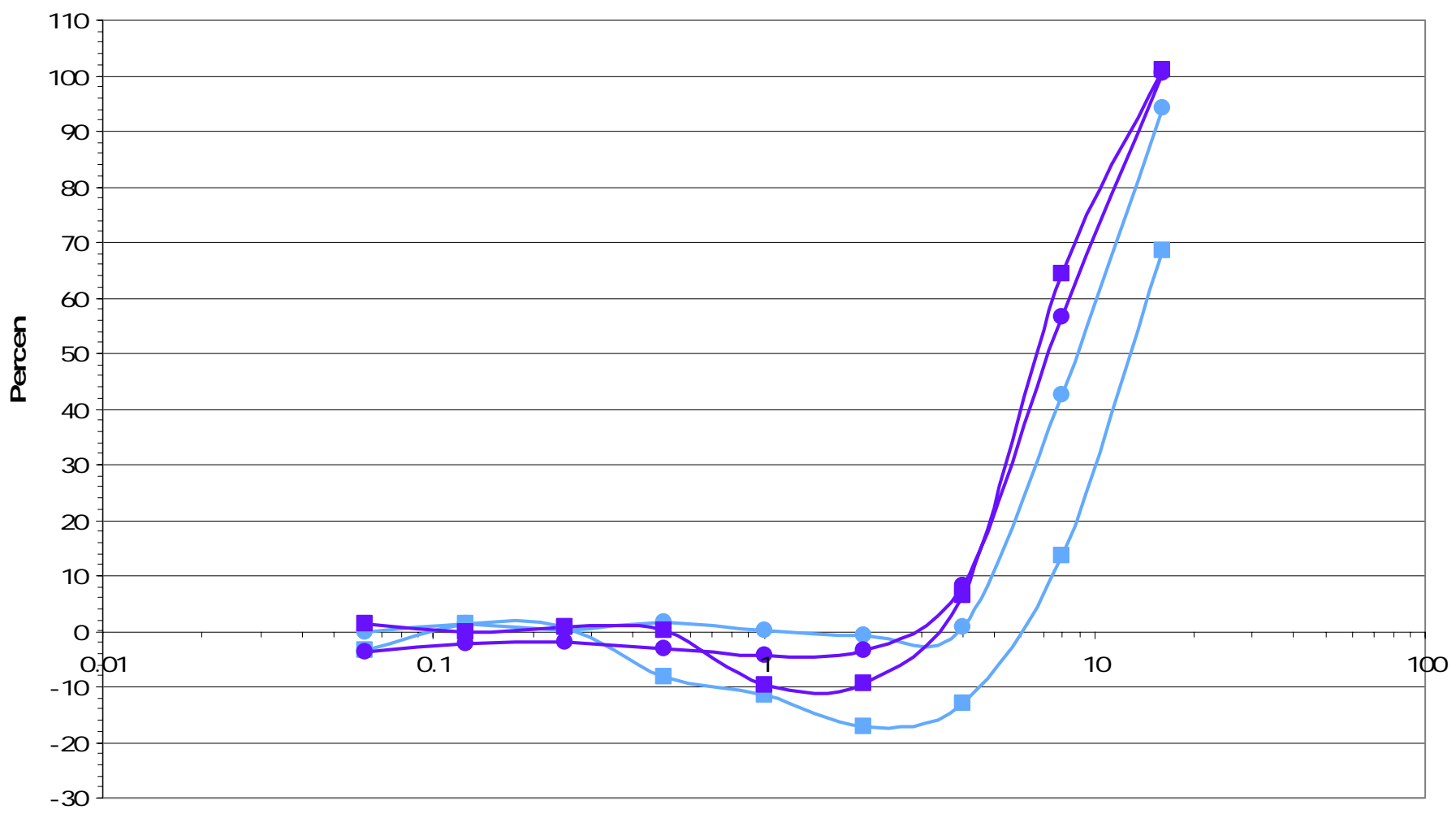

Compound $[\mathrm{C}](\mu \mathrm{g} / \mathrm{m})$ 


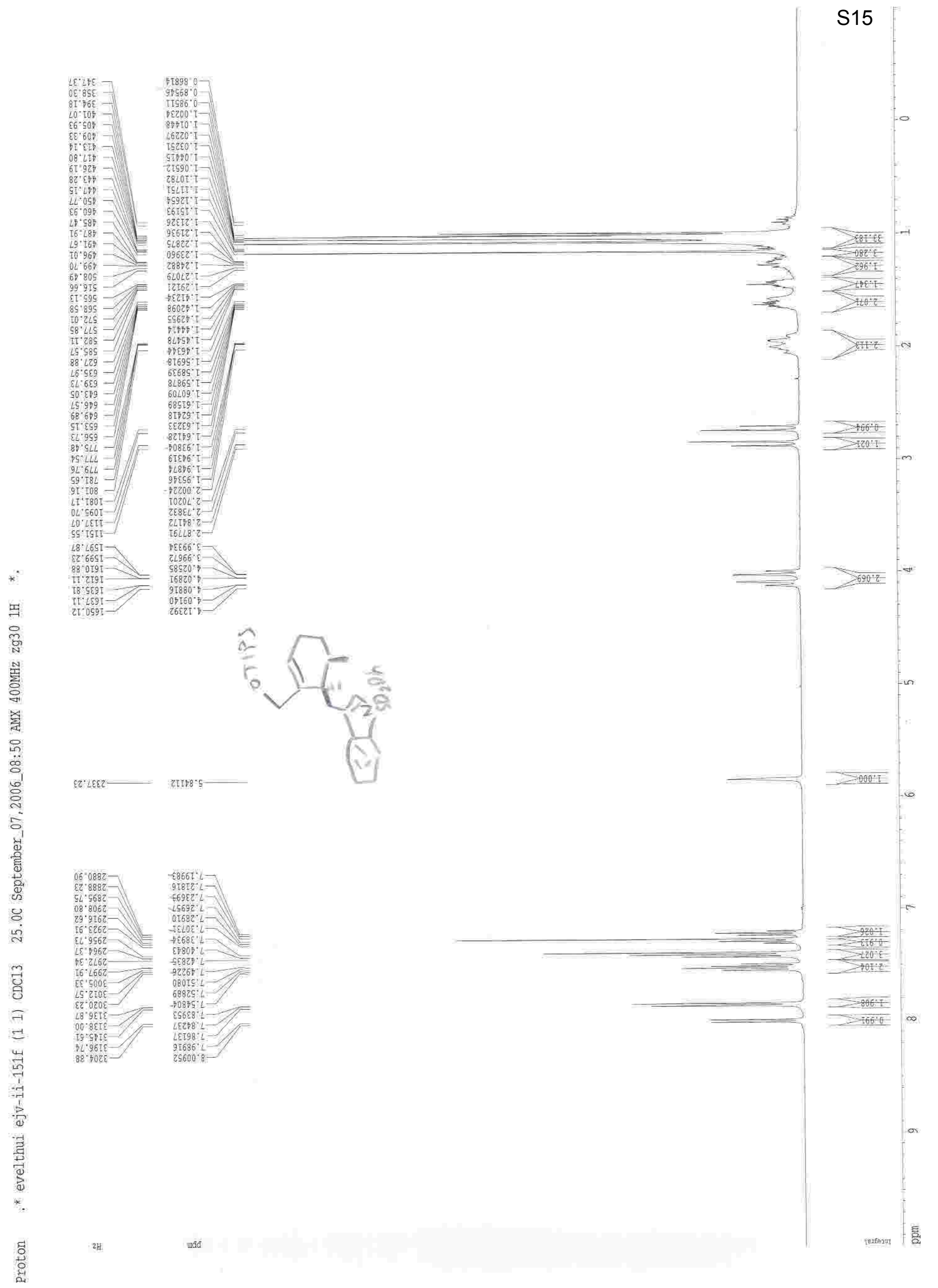




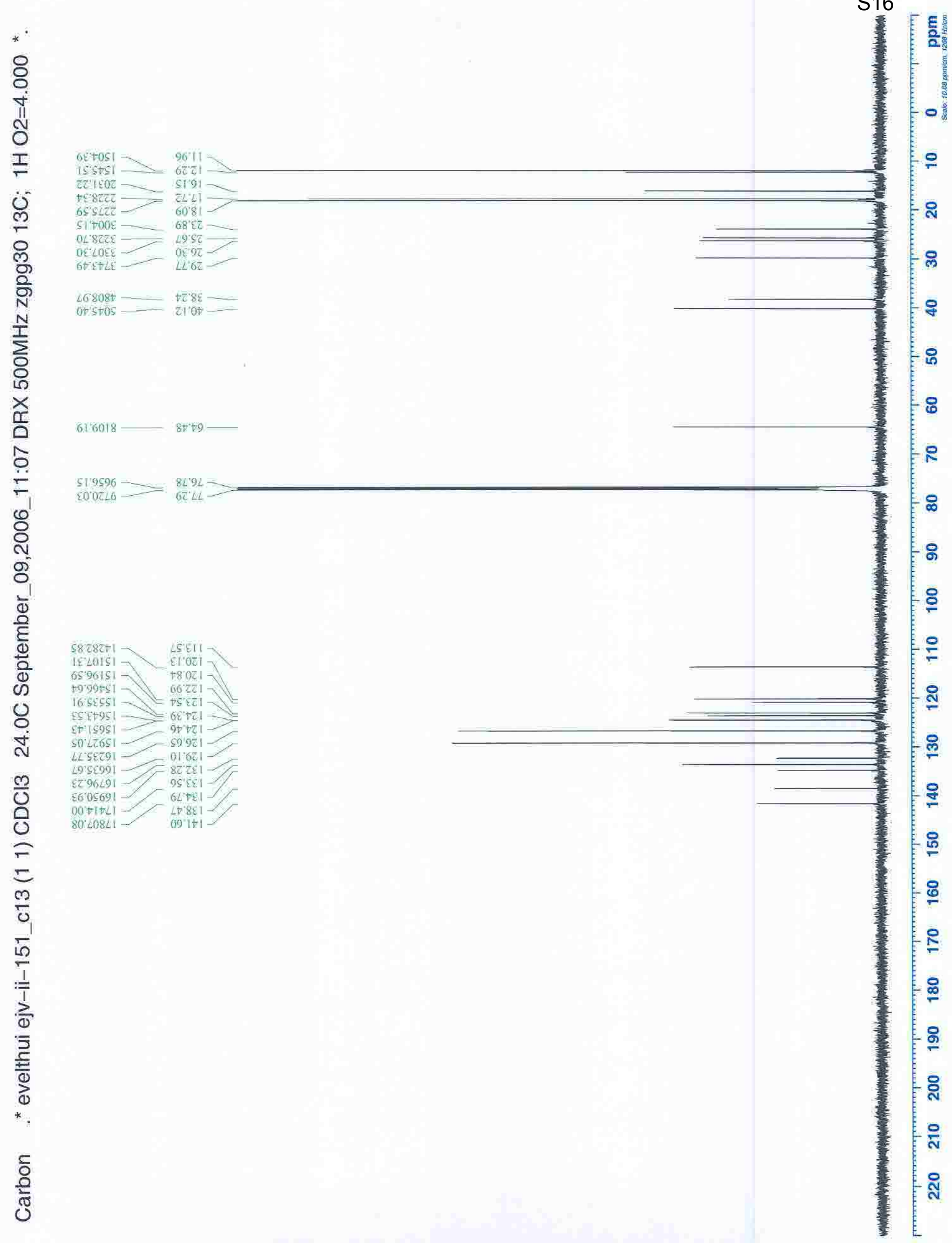


$\forall \rightarrow 0: 1$

S90.

201:-

ELI:-

Zst: - -

E9L : -

E\&ट : -

t9ट !

$66 \mathrm{Z}$ :

२SE:

$99 \varepsilon$ ?

900 .

91t L-

GED L -

ESt : -

$\varepsilon 97$ :

$8 \angle t L$

009 : -

ZIS'L-

$\angle 19{ }^{\circ}$ -

989 :

tog.

$9012-$

ELLZ

$0 z+2-$

$928 z$

$\angle 192-$

ट॰9 ट-

$879 z-$

$\varepsilon \angle 9 \mathrm{Z}$

$8 \mathrm{CL}$

$\nabla ट 9^{\circ} \mathcal{E}$

०७९'

$\succ 99 \varepsilon$

6698

$91 \angle \varepsilon-$

120

$880 t-$

$960 t-$

$90 \mathrm{H} t-$

$\nabla 11+t-1$
$2+60-$

$\checkmark 60$ L

$692<$

$0<2 L$

¿८ट $L$

$20 \varepsilon<-$

$9 เ \varepsilon<-$

$98 \varepsilon: L$

$Z L E L$ -

$927 L$

$8 E T L$
$19 D$

$\triangle Z G L$ -

$\angle E S^{\prime} L$

$6+9 ' L$

$\varepsilon \rightarrow 8: L$

$1.28 \mathrm{~L}$

$888 \mathrm{~L}$

$286 \%$

$966 \mathrm{~L}-$
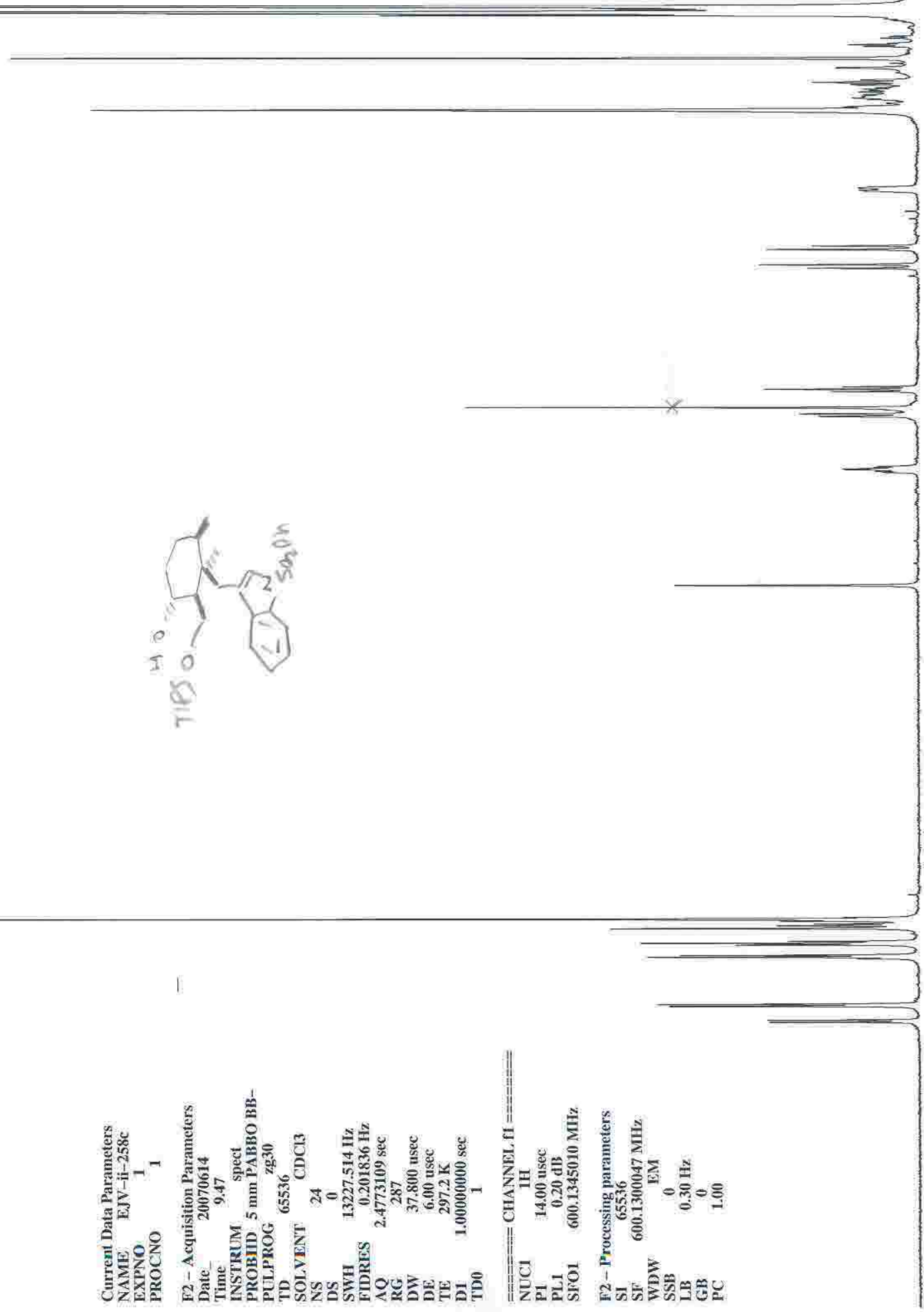

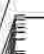

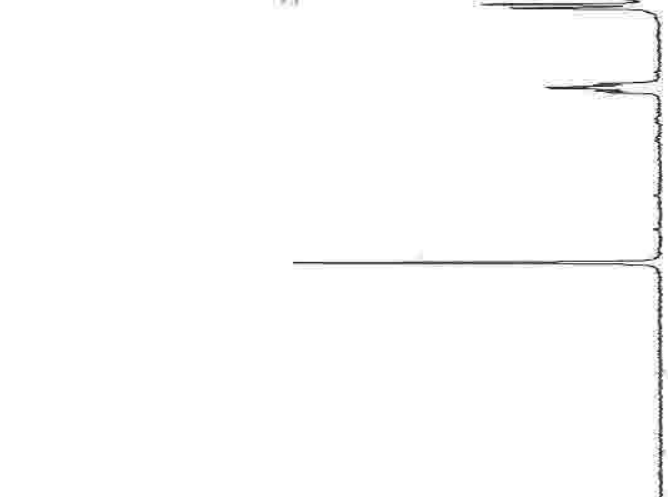




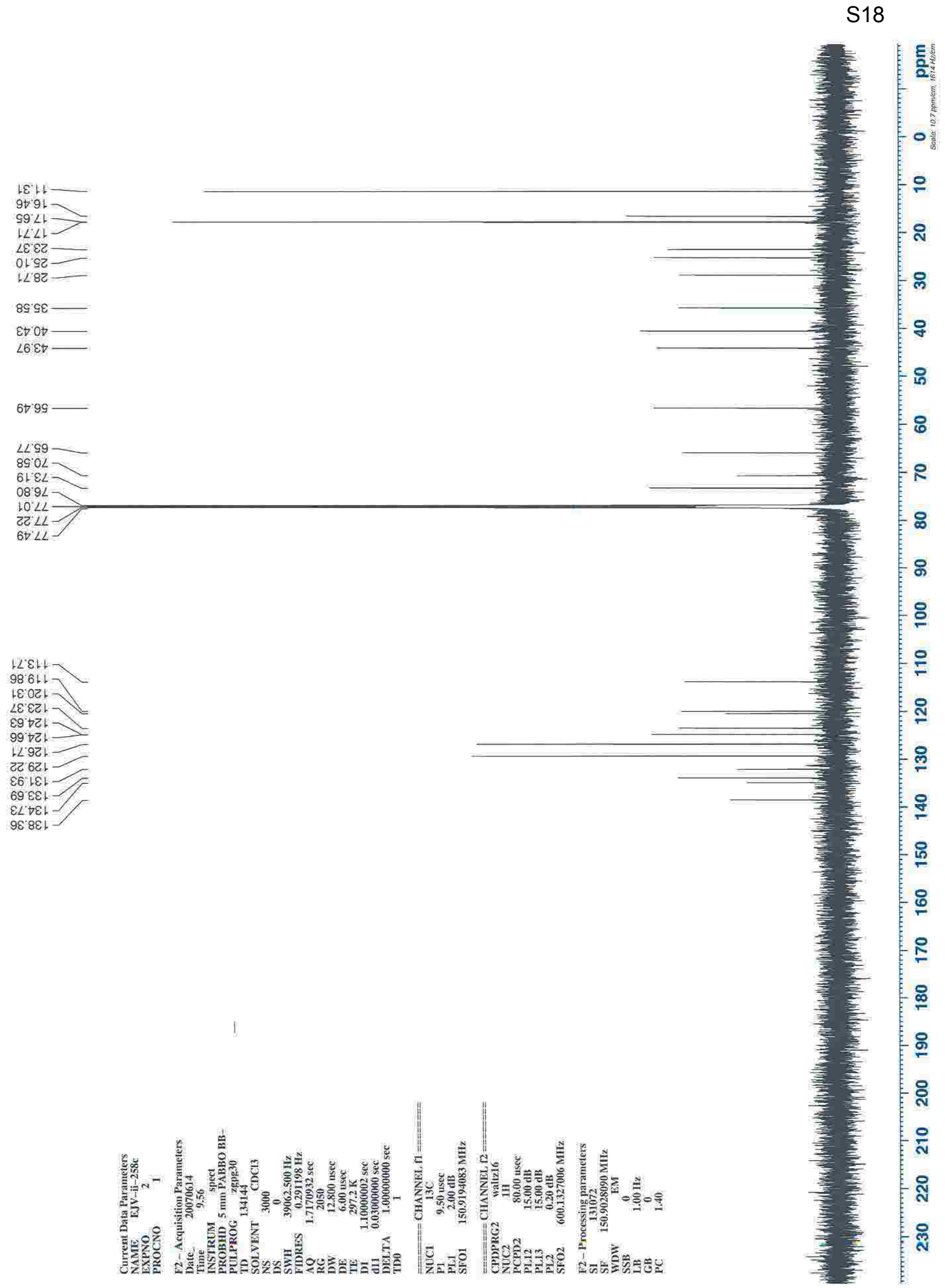




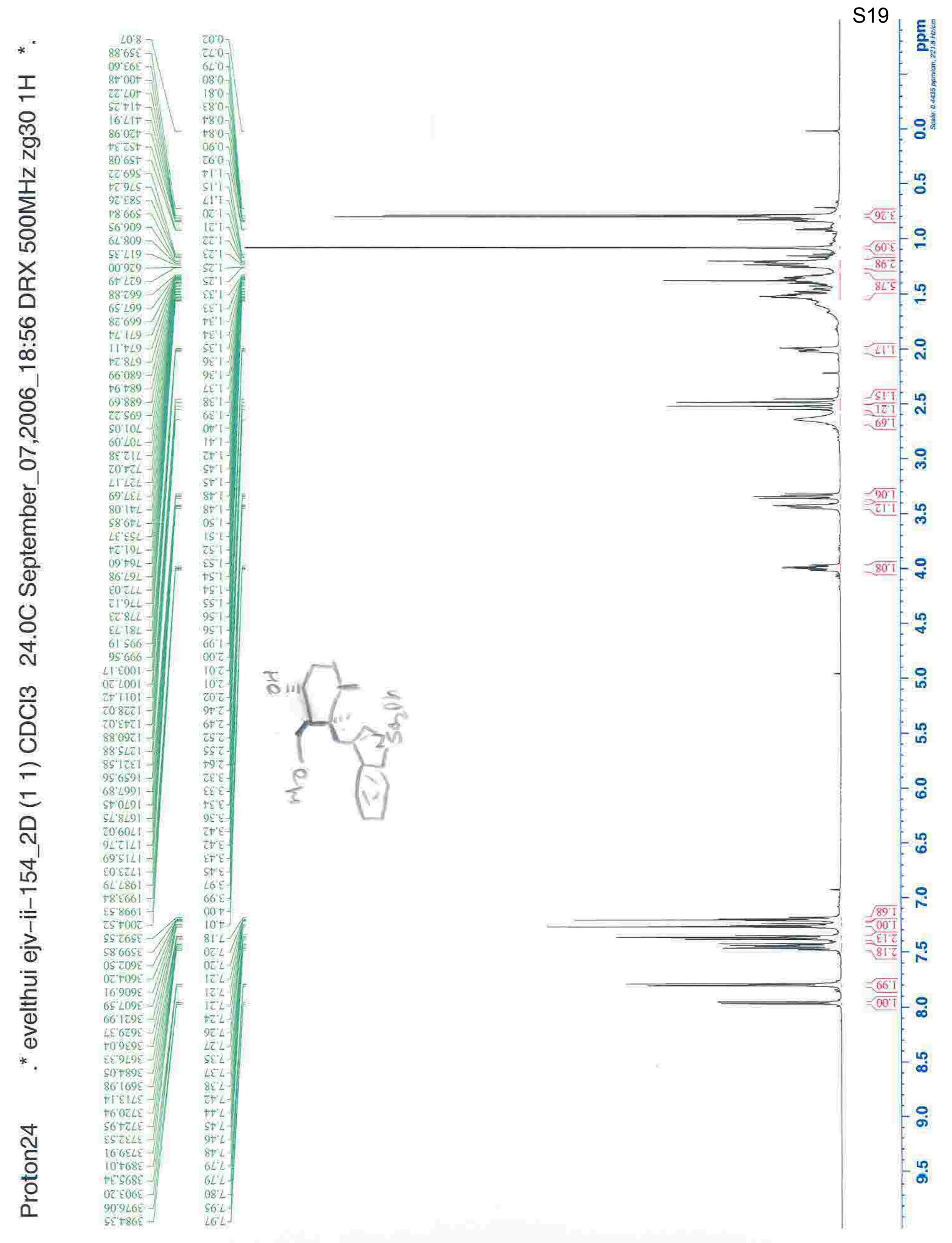




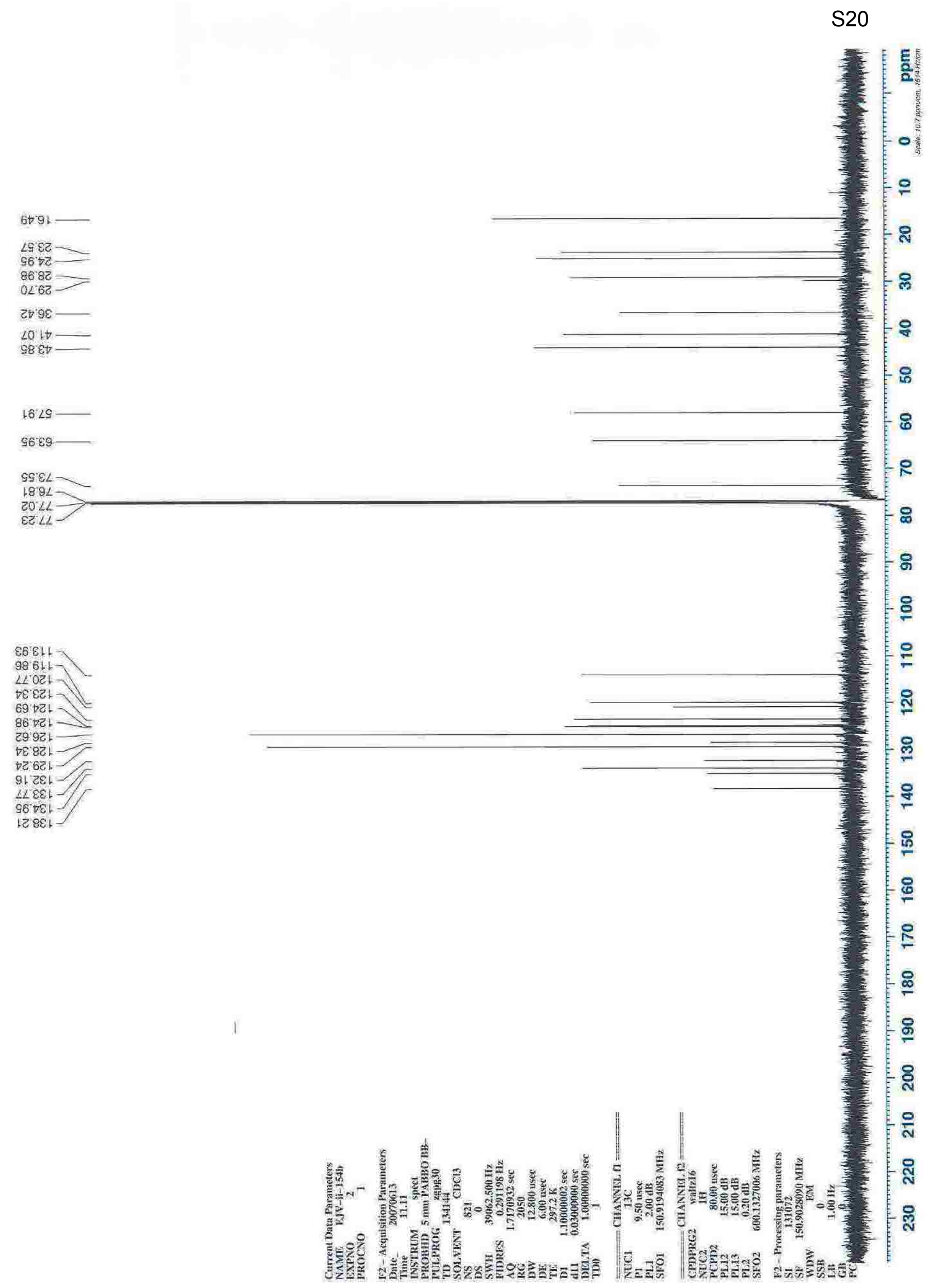




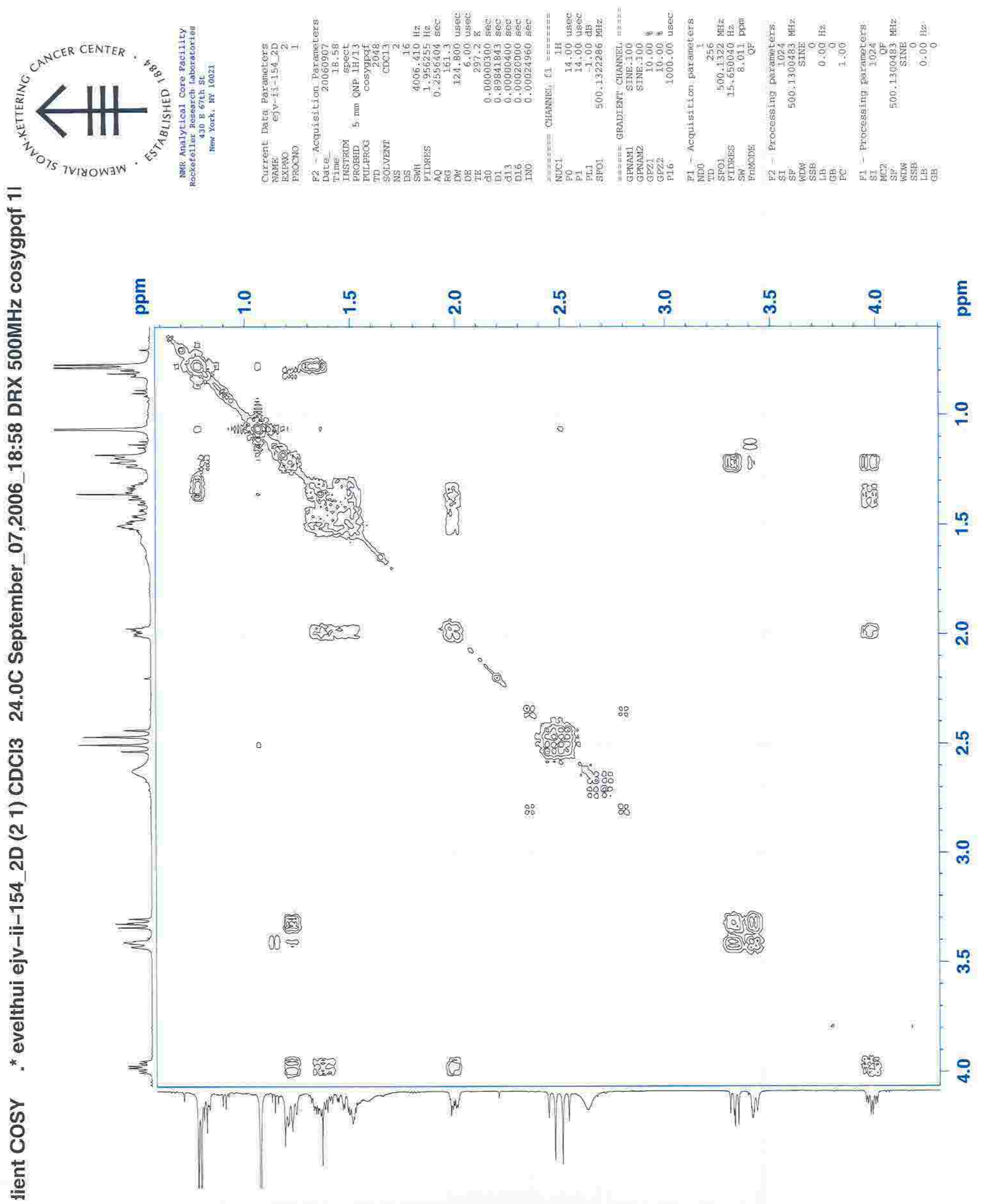




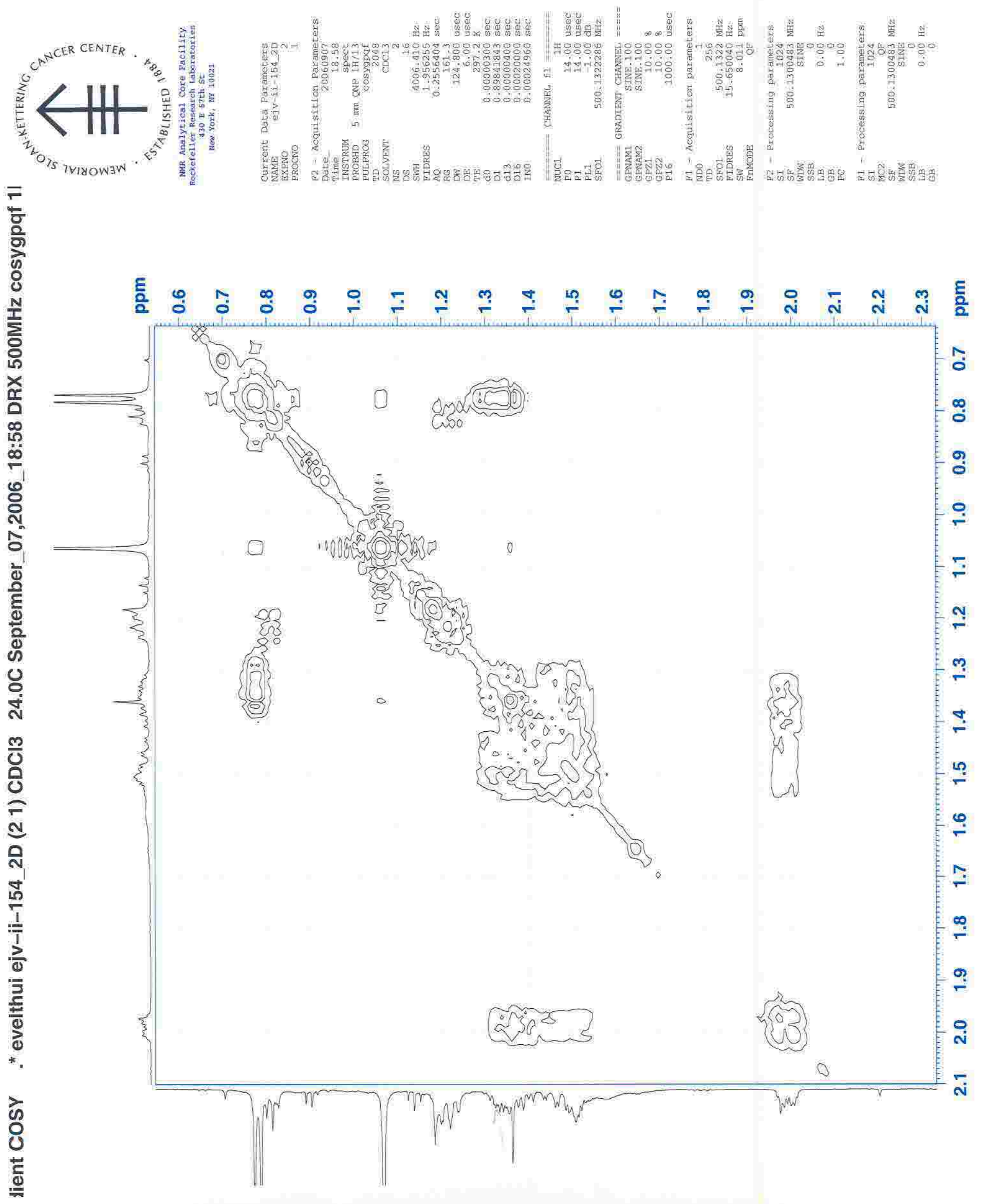




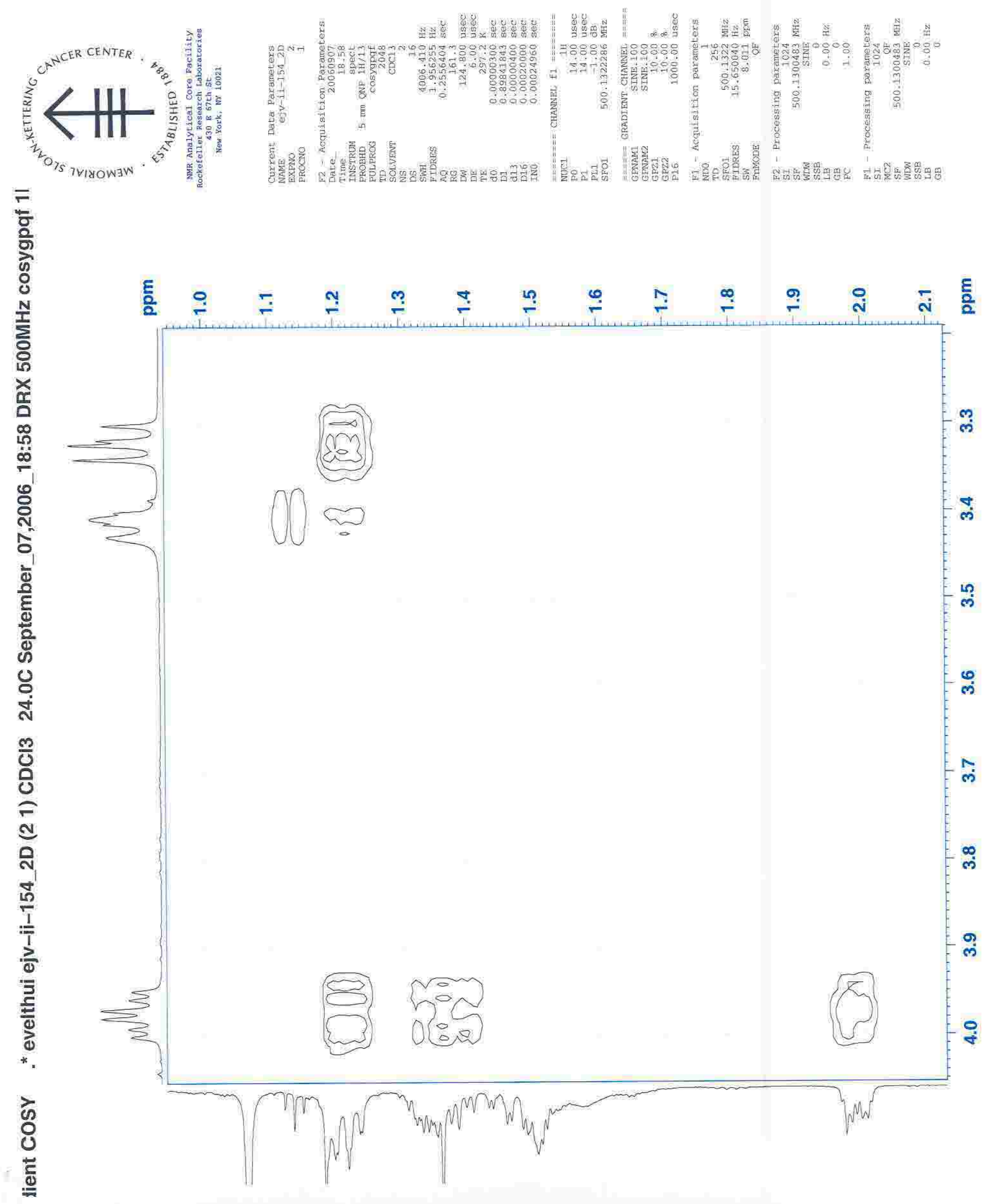




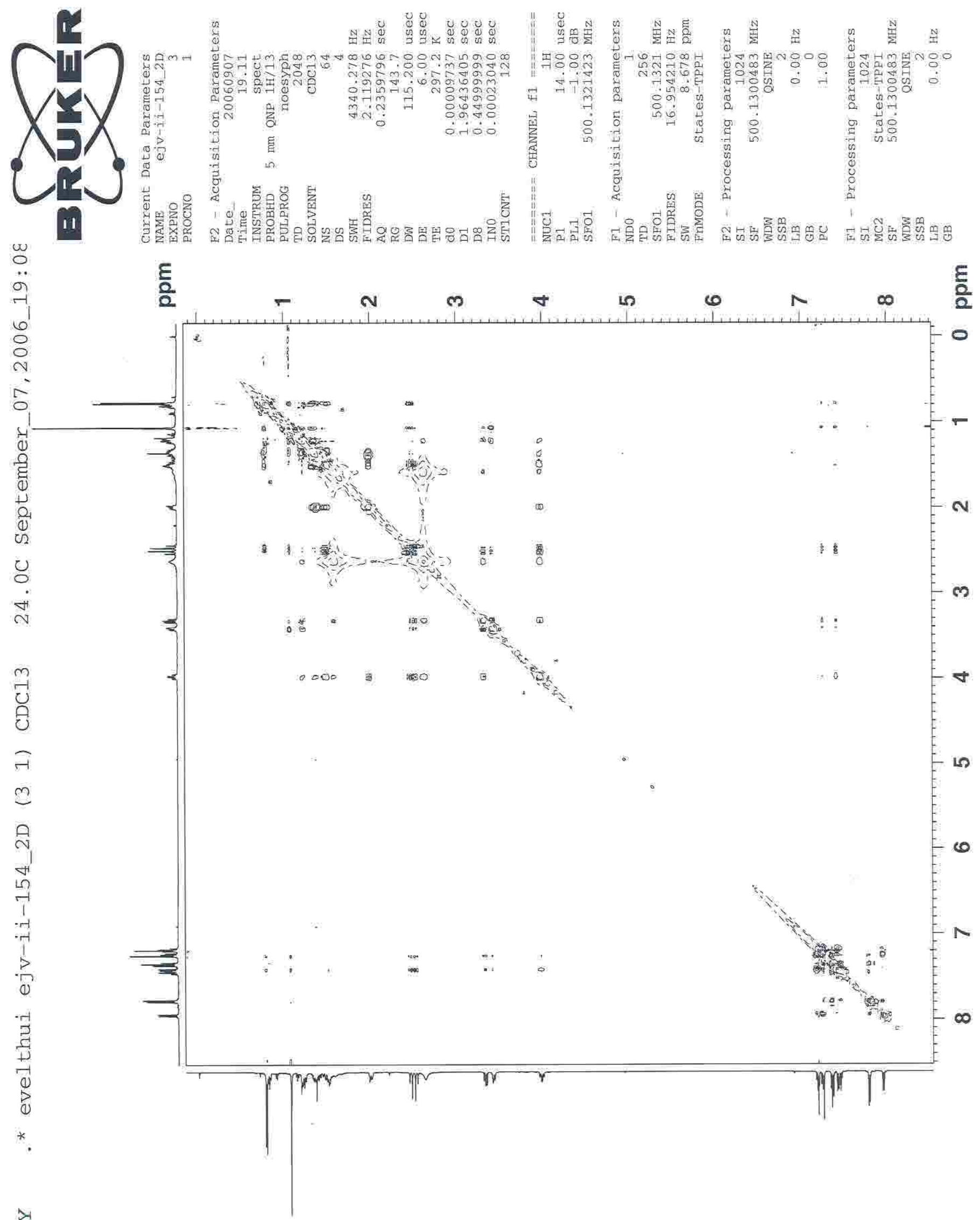

7
0
0
II
0
$z$ 


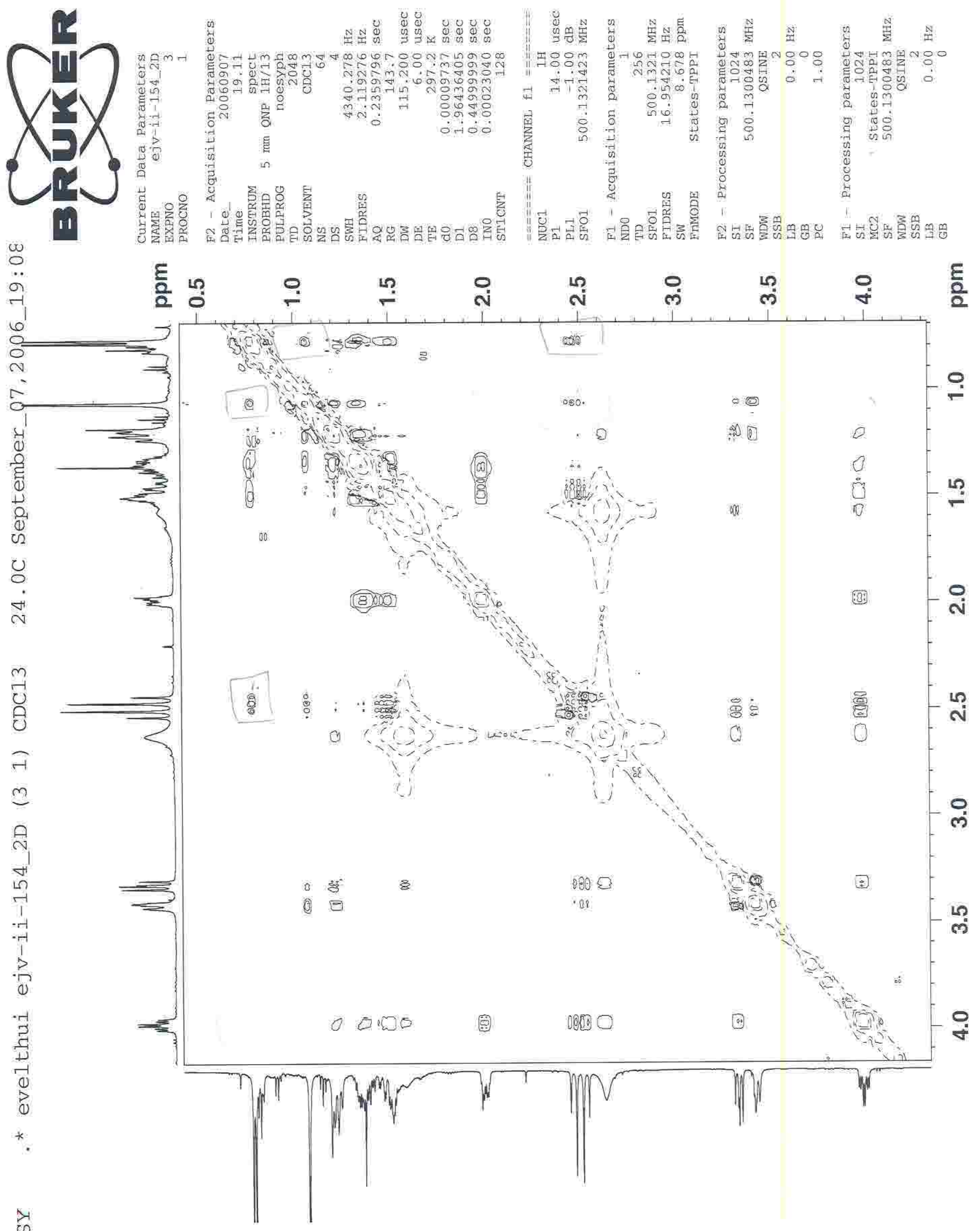




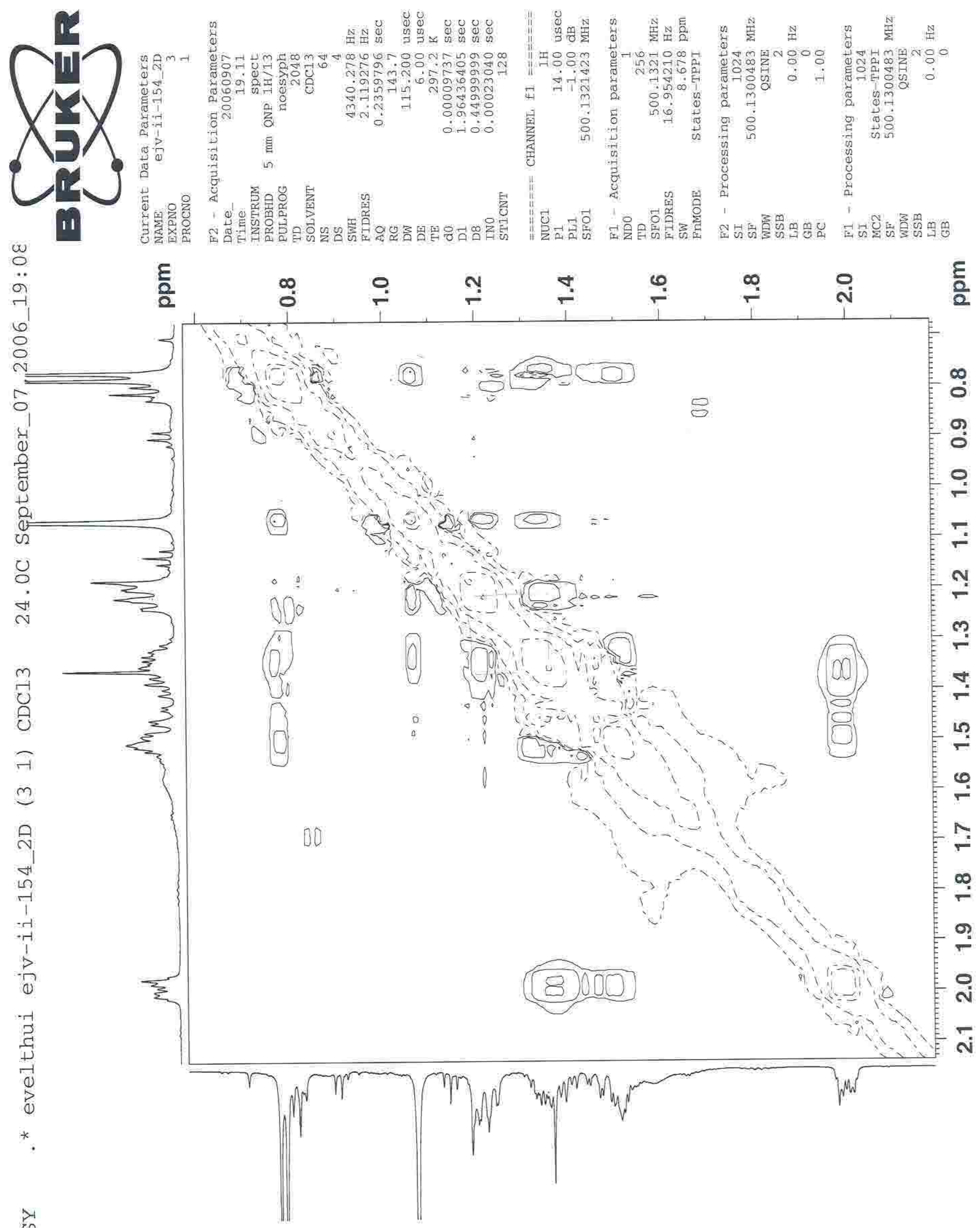




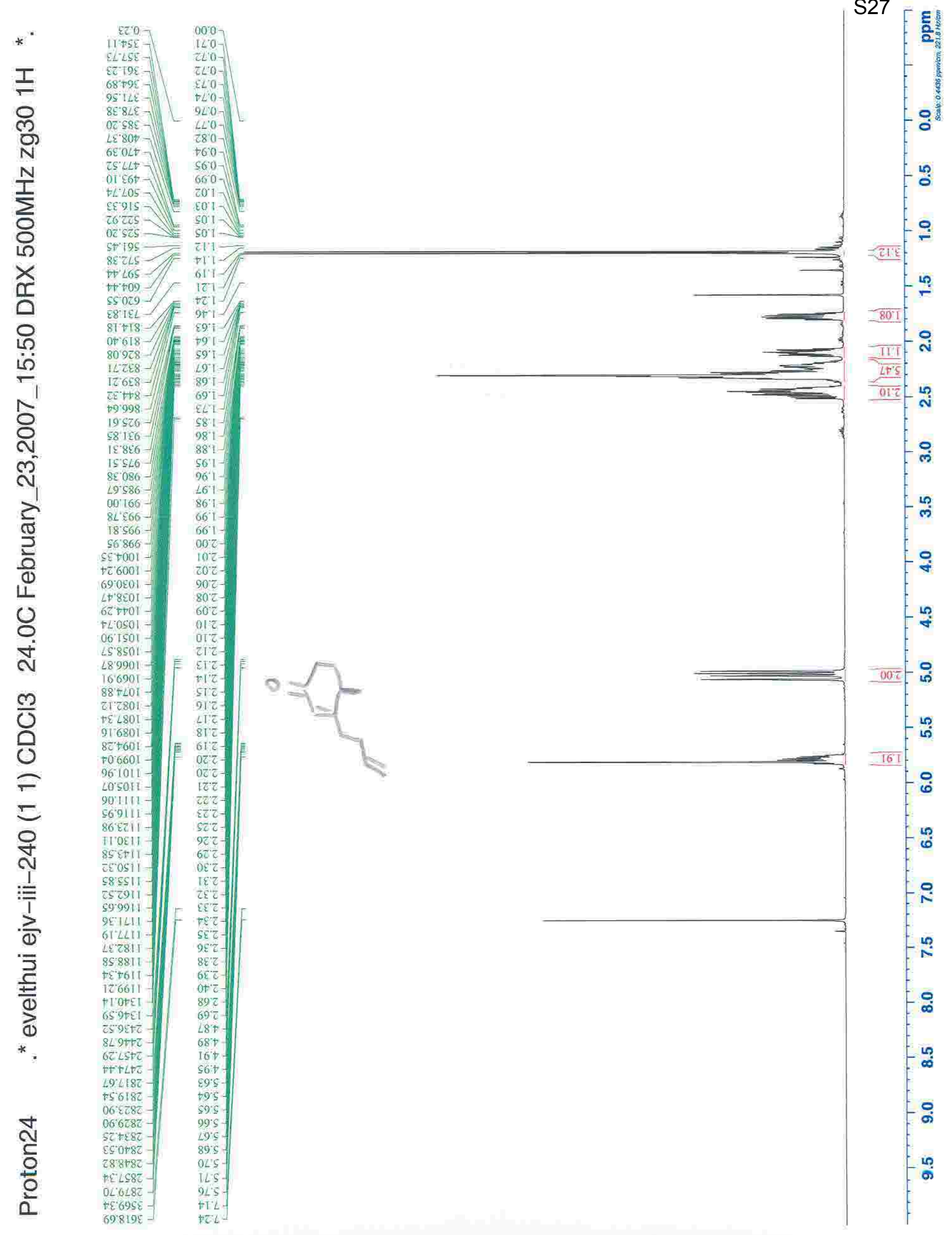




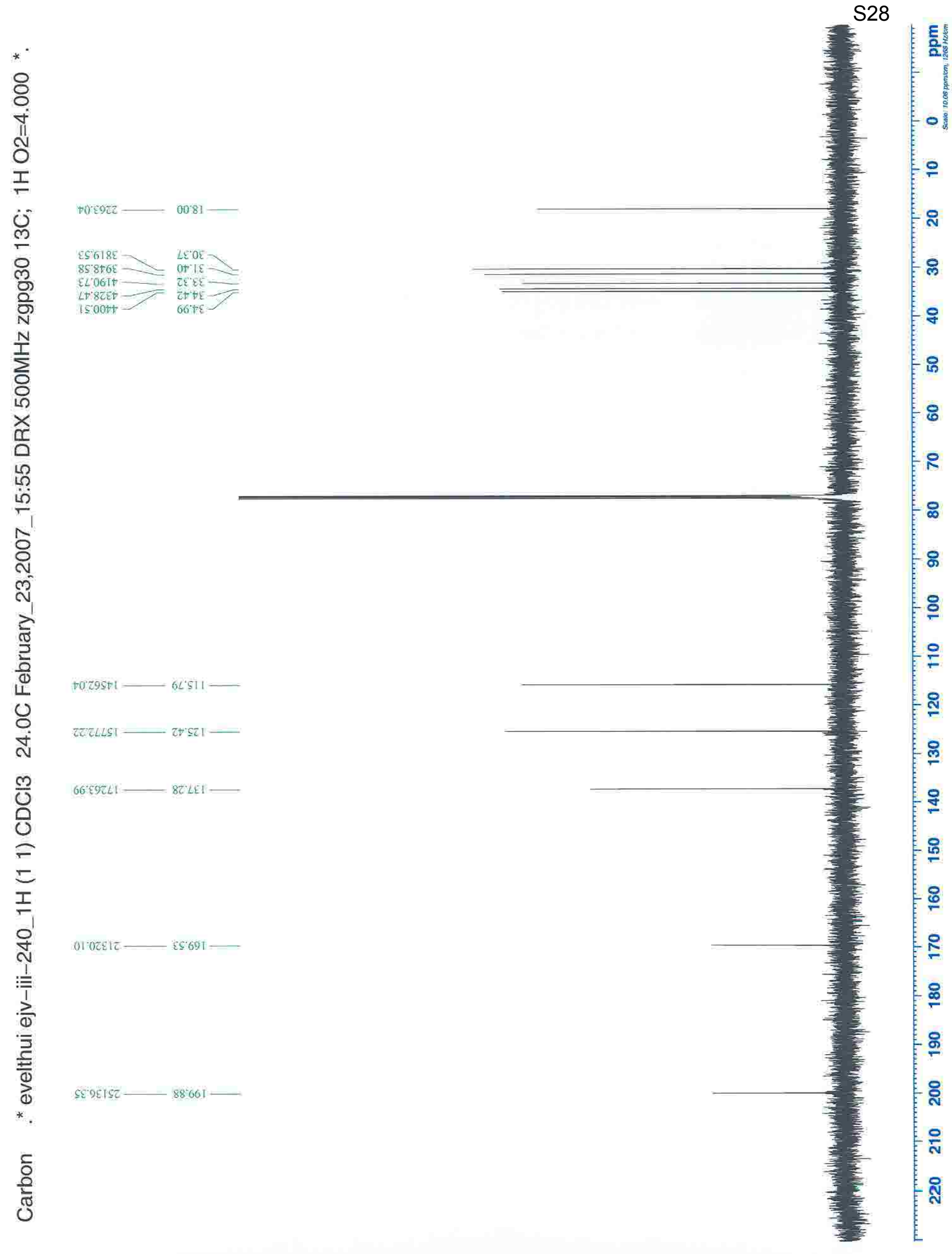




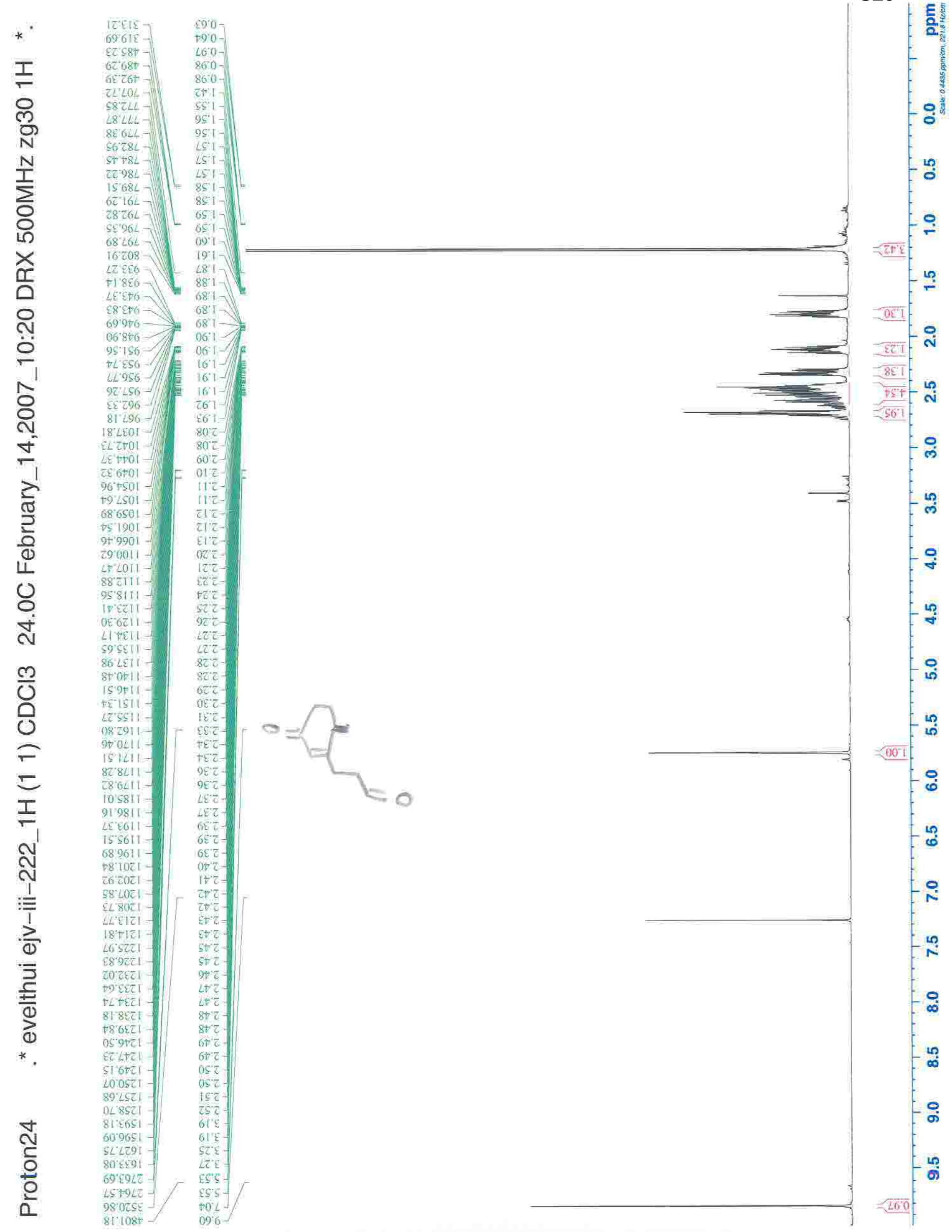




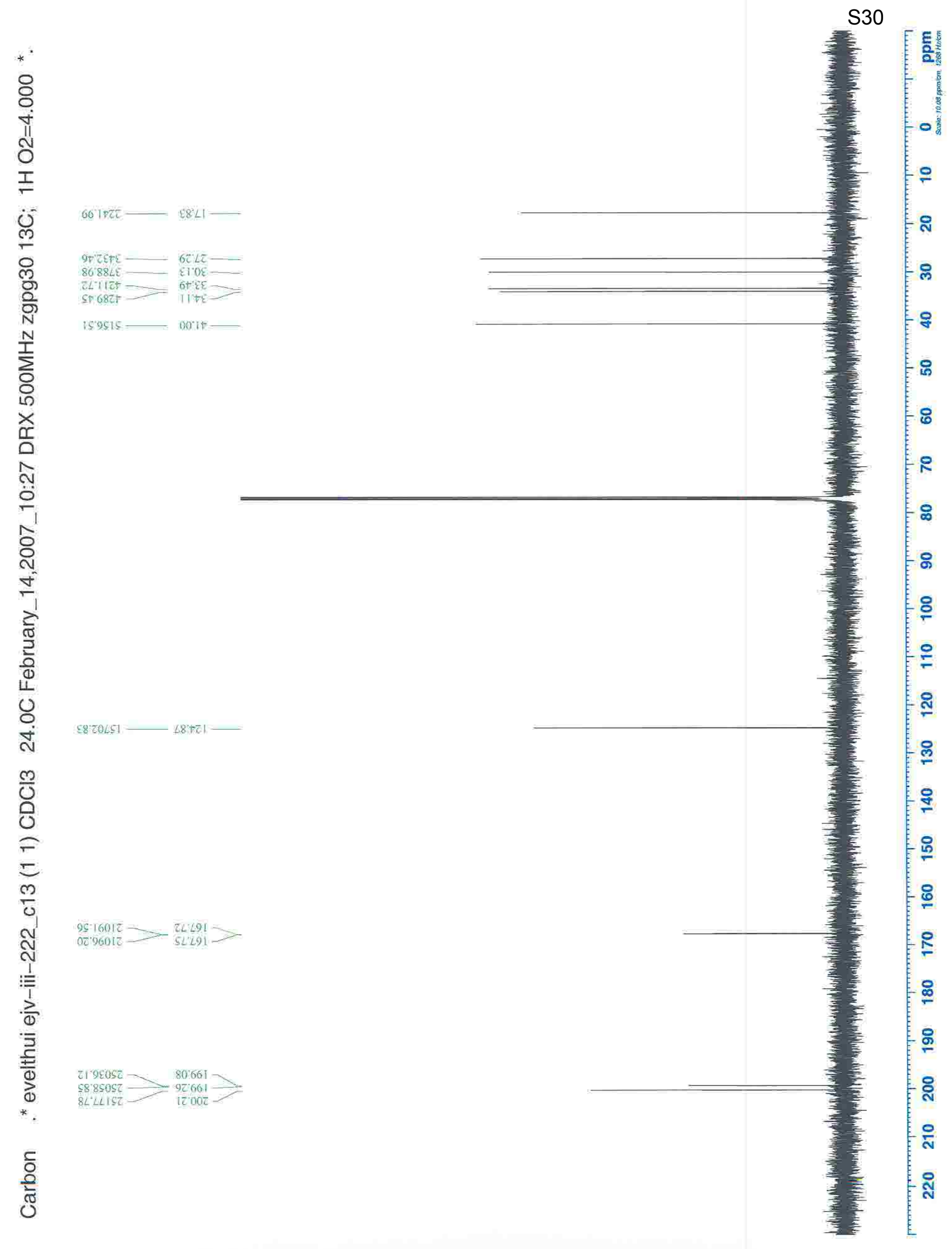




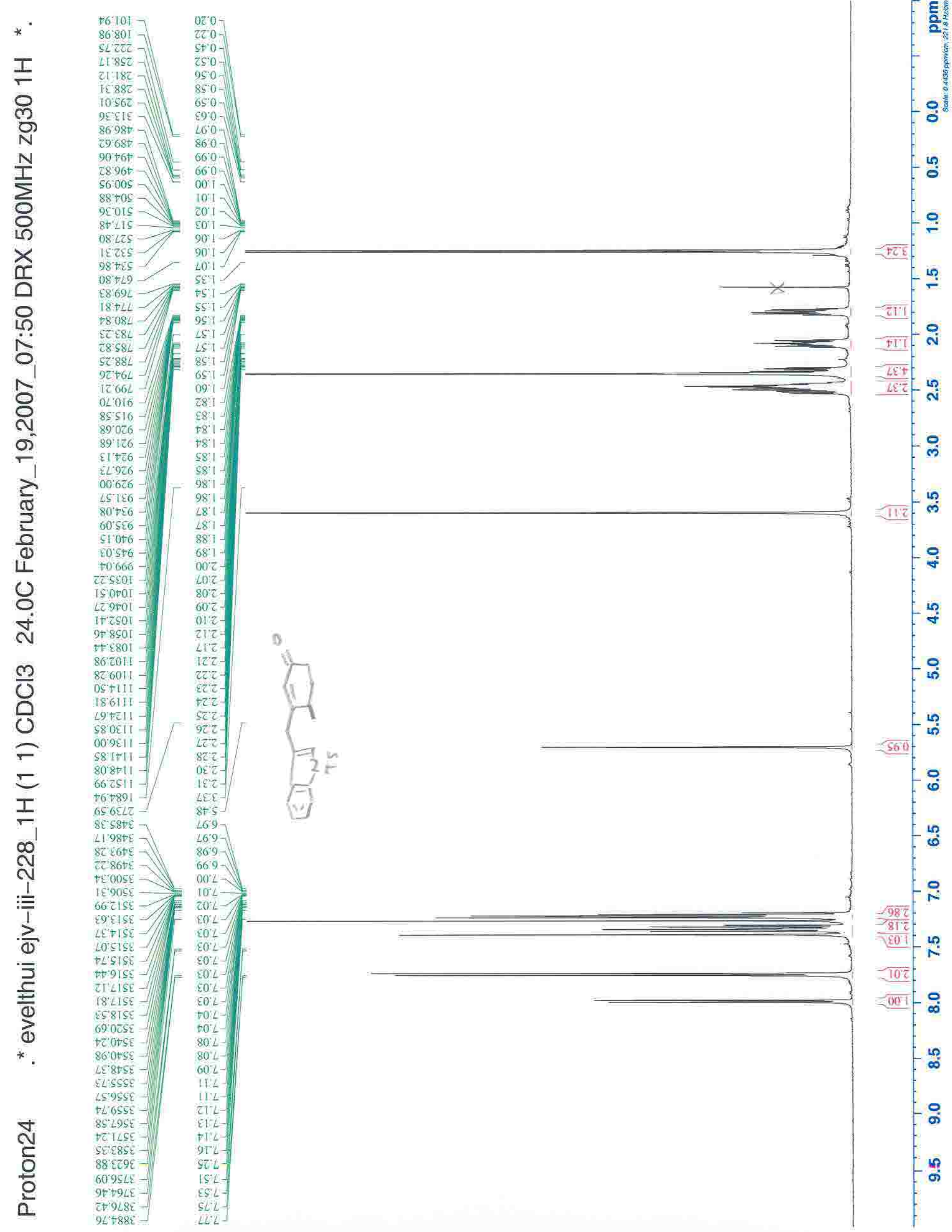




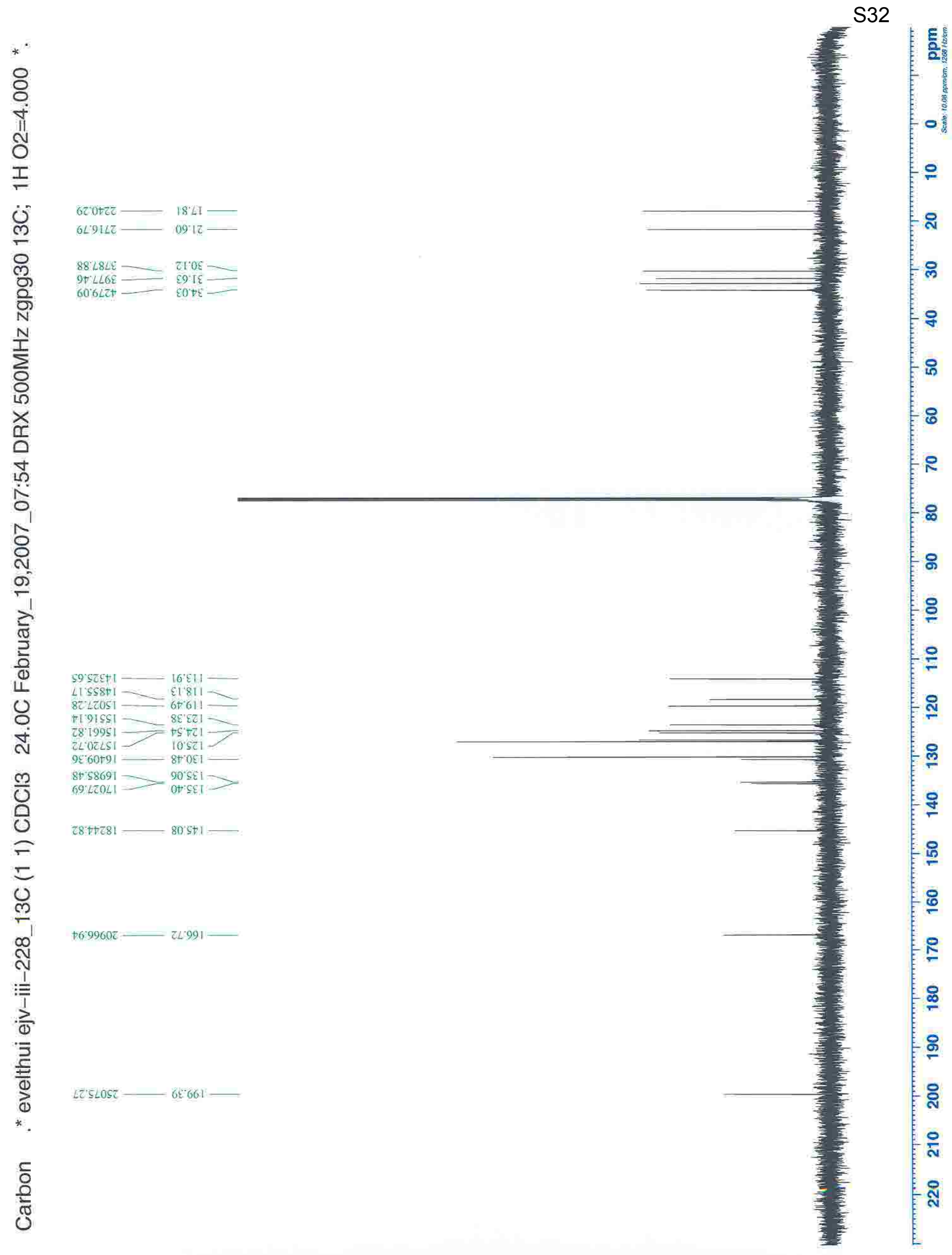




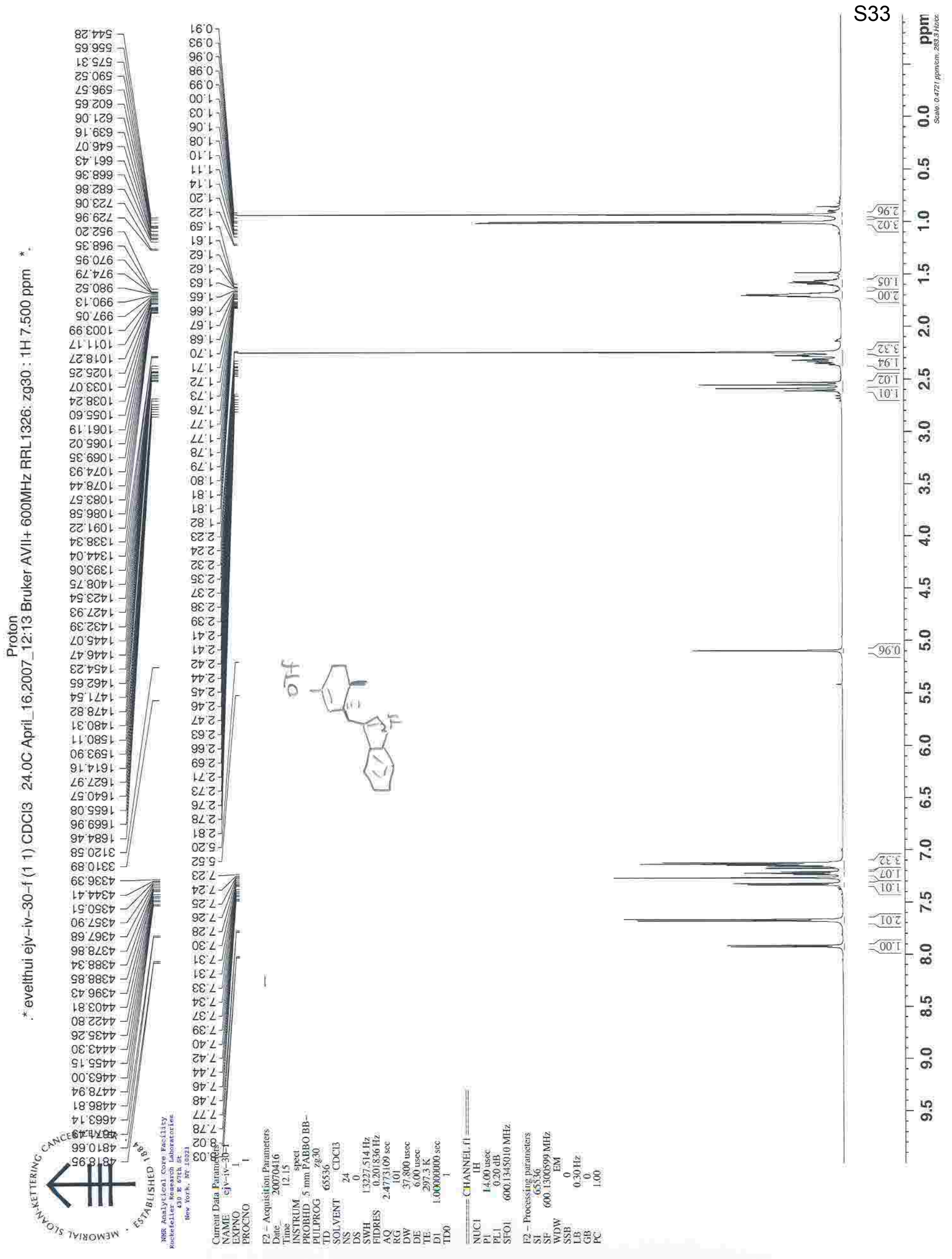


ह

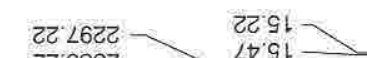

$2291>$

टZ $9 \& \varepsilon Z$

$\angle \nabla 91$.

$99.972 \varepsilon-$

LG'L2

\&L99ZE

11.92

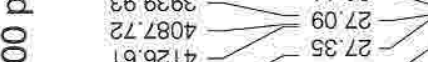

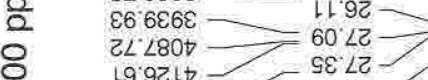

$\angle 6 \angle 29 D=\angle 908$

$\angle 0^{\circ} 8 \varepsilon$

09.9889

$208 \varepsilon$
-1068

0

.

o

긍

लิ
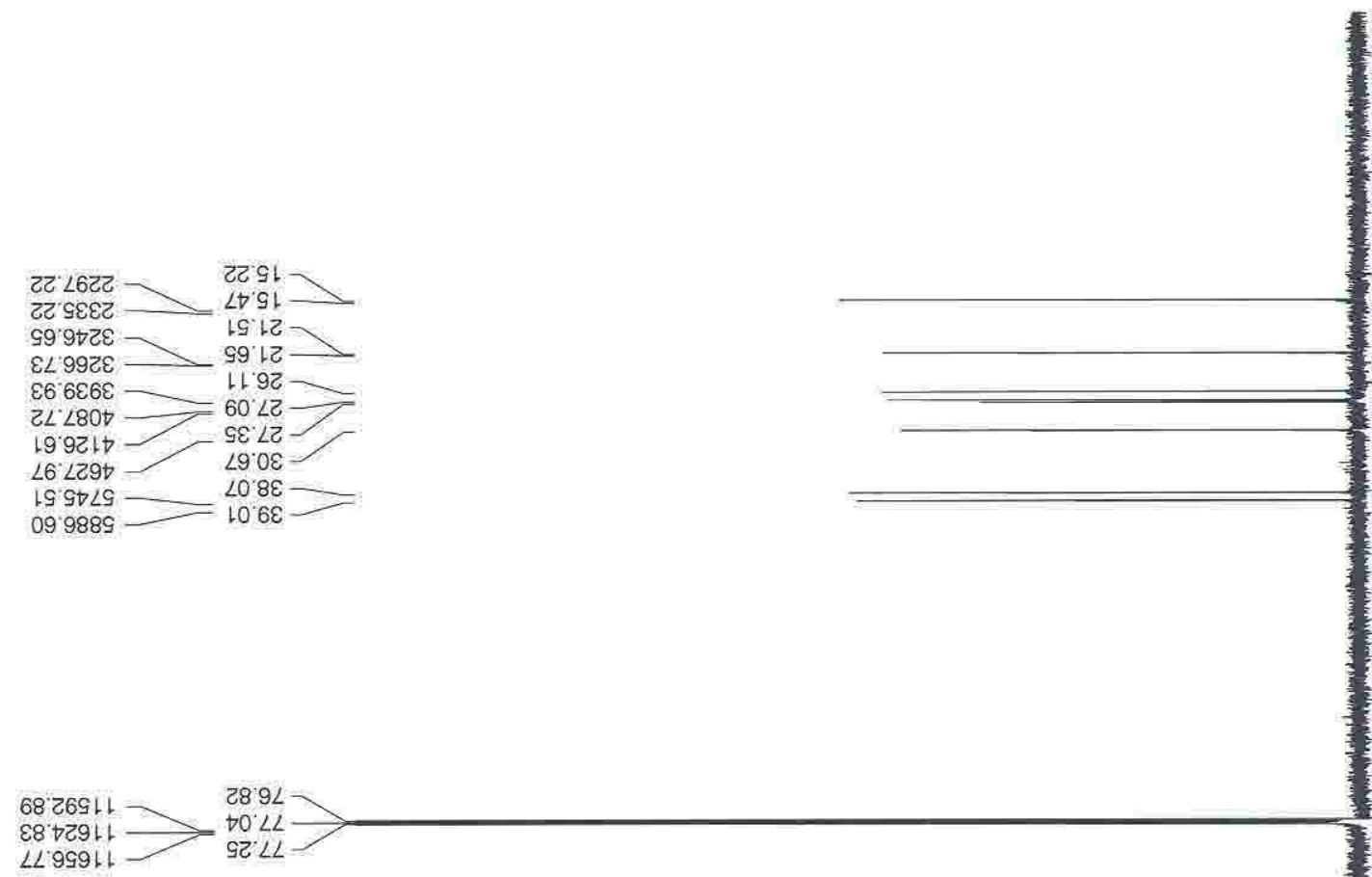

$=\frac{1}{2}=$

$E$

ำ

-

웅

요

8

R

$\infty$

8

8

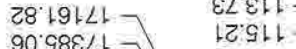

$96 \rightarrow 0 \angle L L$

$6 Z+\angle 8 \angle h$

909208

$21<1+81$

S! $9 \triangleright \varepsilon 81$

$0 Z 88981$

66.10881

$\triangle 0 L 921$

$0062161-$ OL LZL

$062696 \mathrm{~L}-5862 \mathrm{~L}$

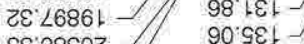

¿E $08 \varepsilon 02$ -

$\varepsilon<96802$ - 16 tot

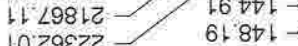

당

ปั

m

$\widetilde{F}$

ㄴ

ग़

$\frac{7}{1}$

$\frac{5}{\frac{1}{5}}$

ONCER CENTER.

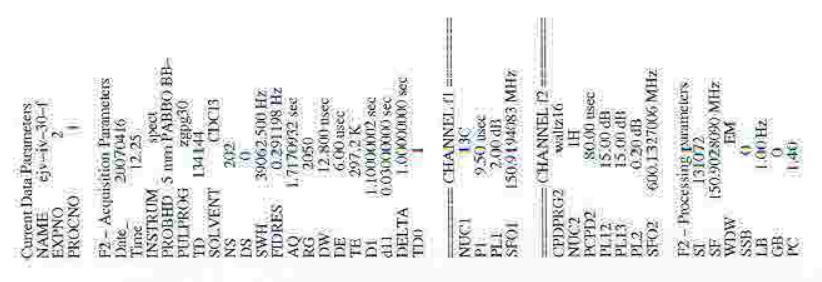

ק 


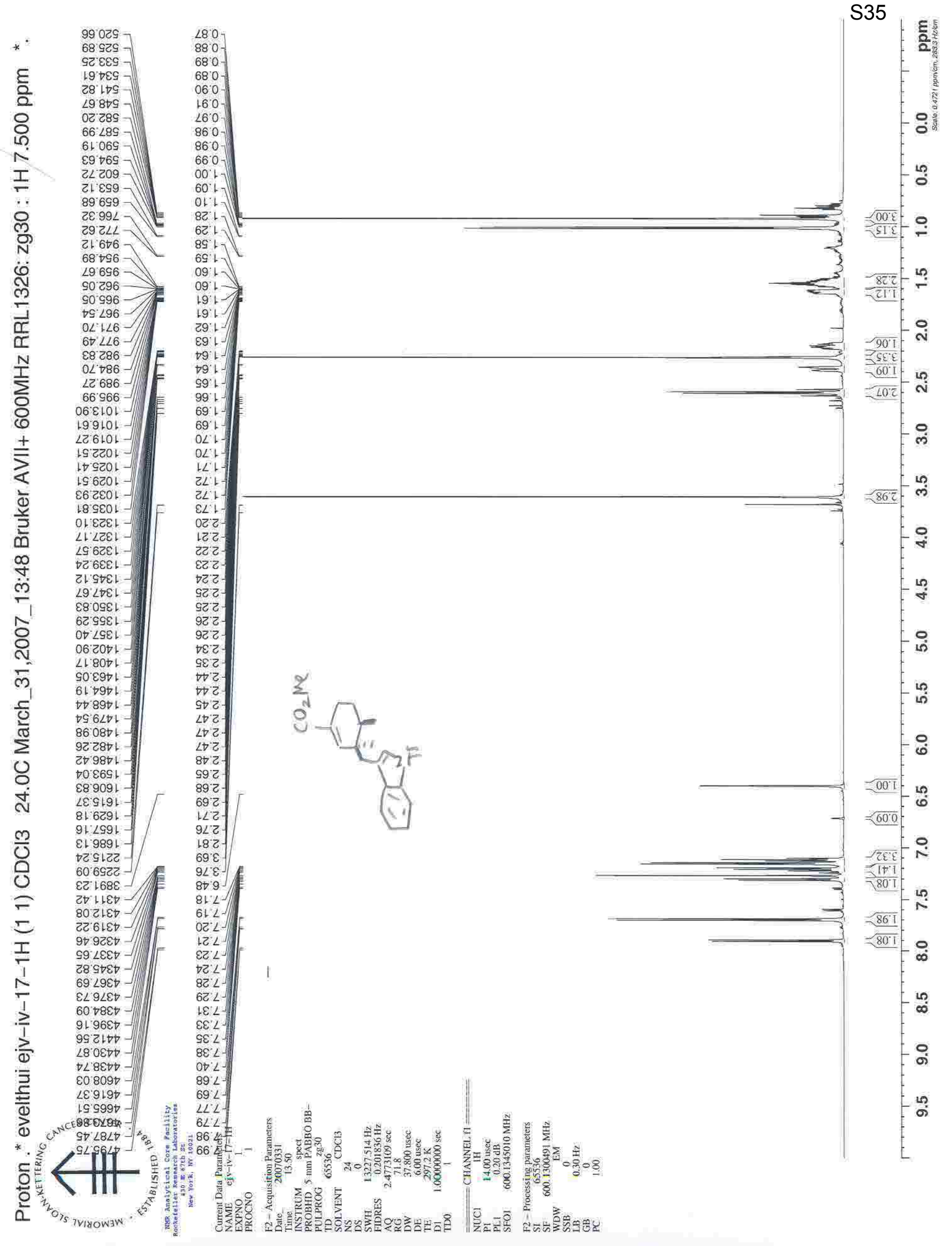




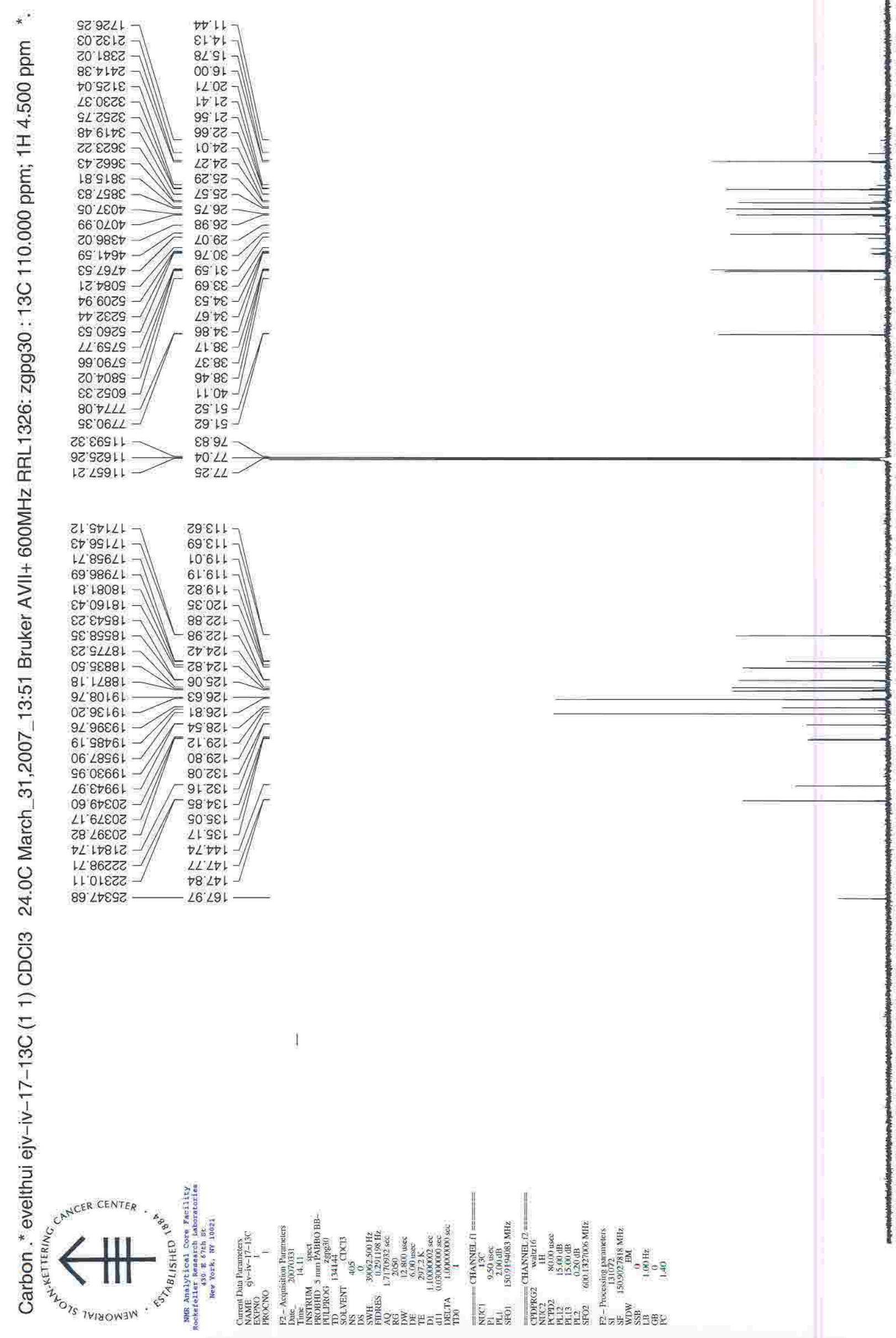




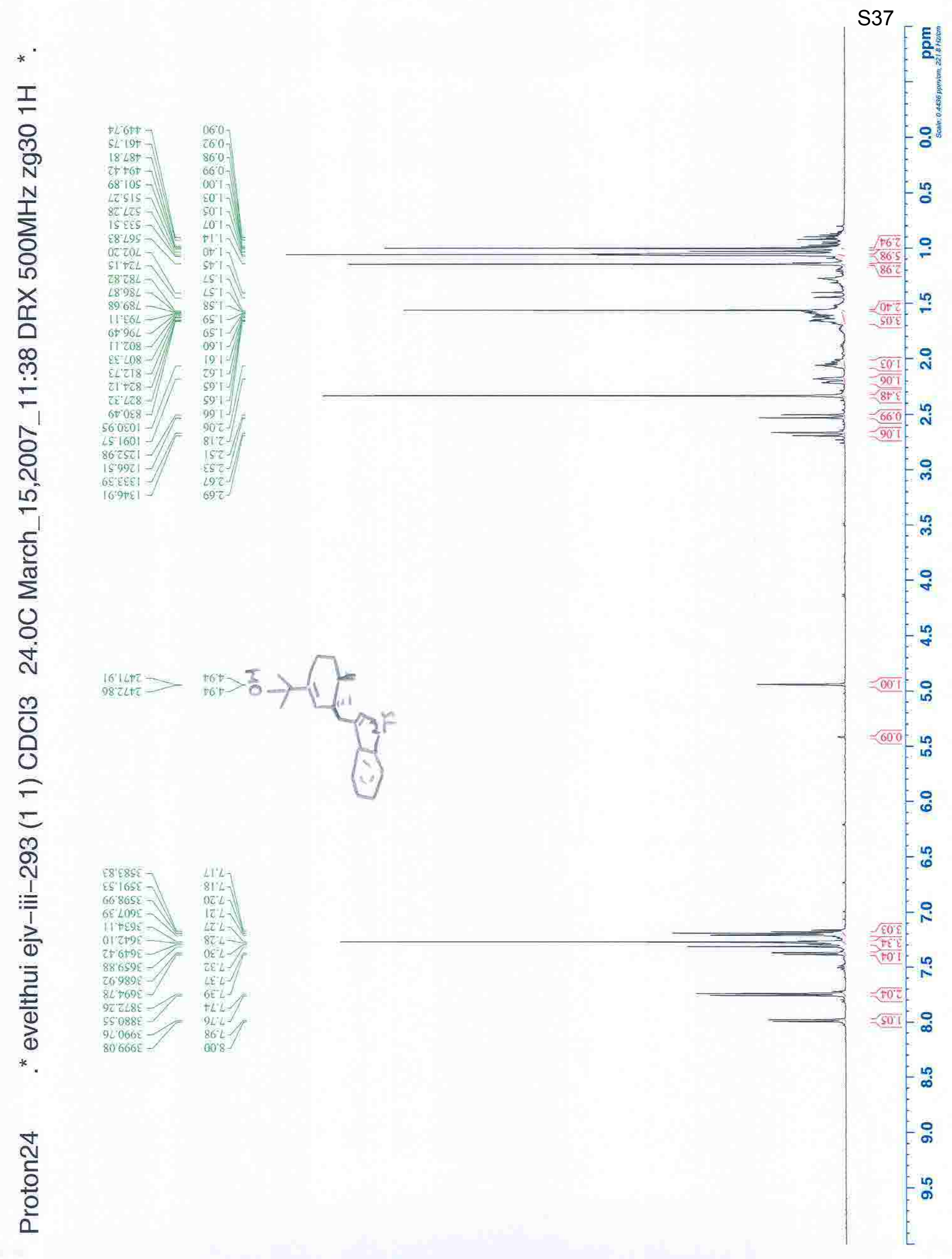




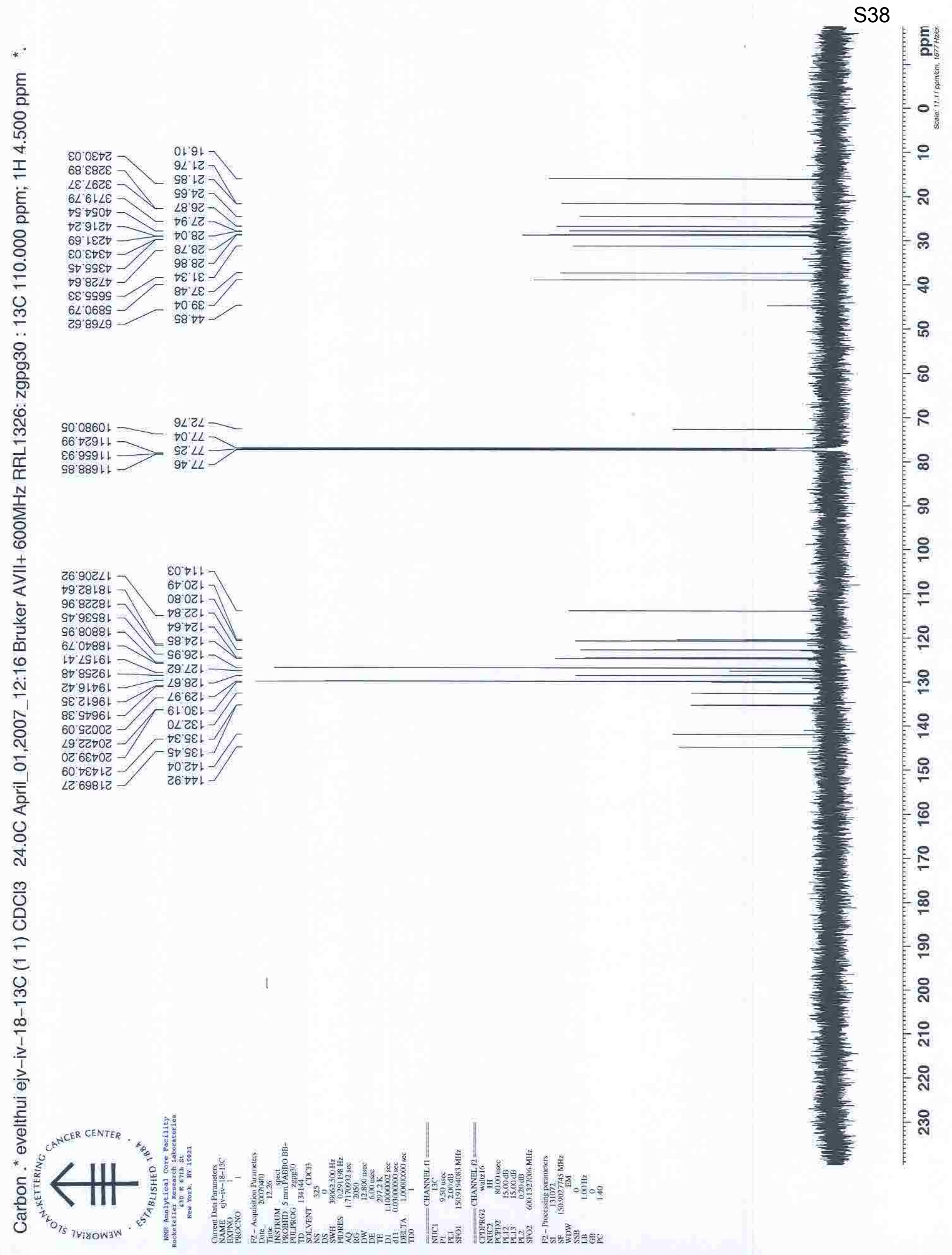




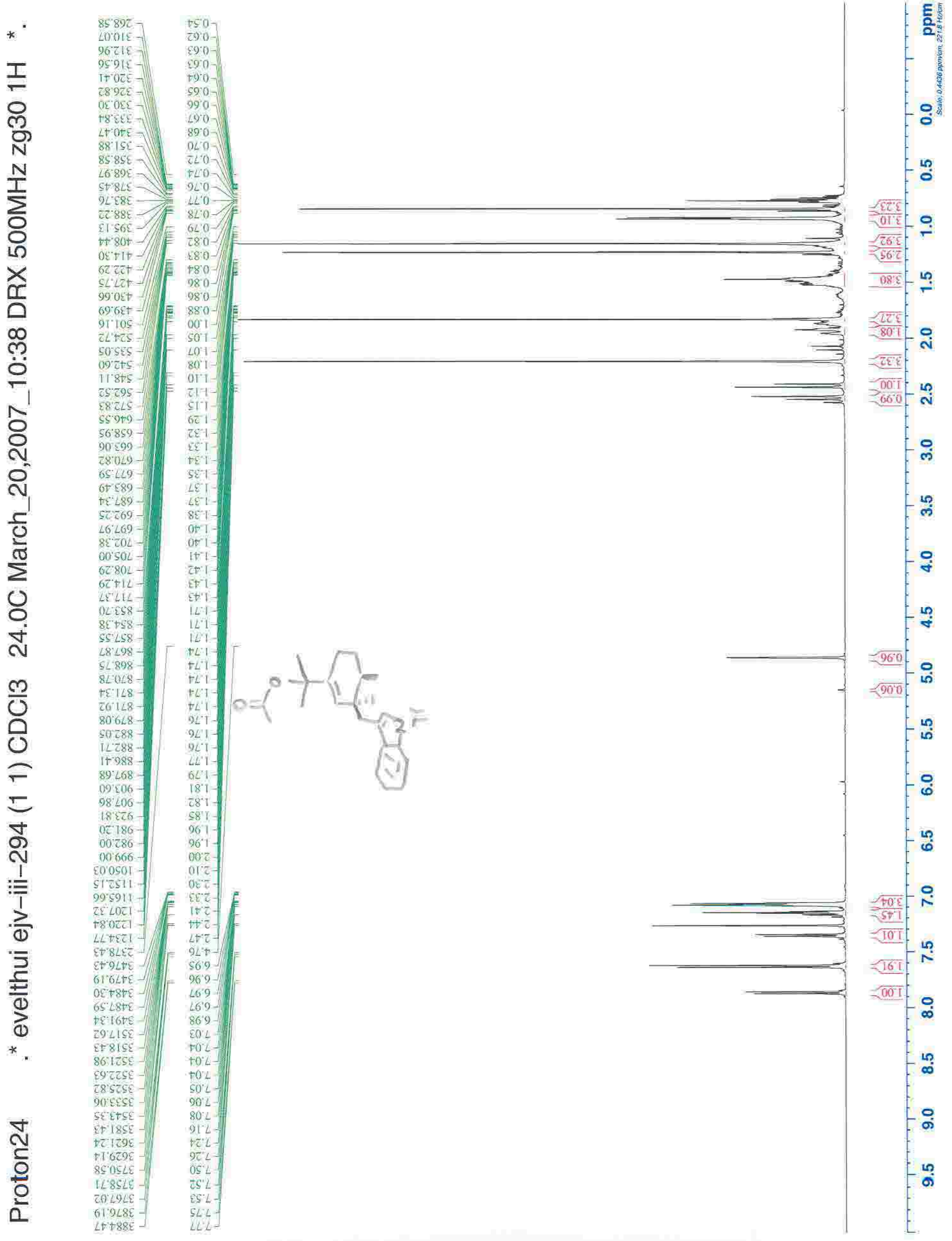




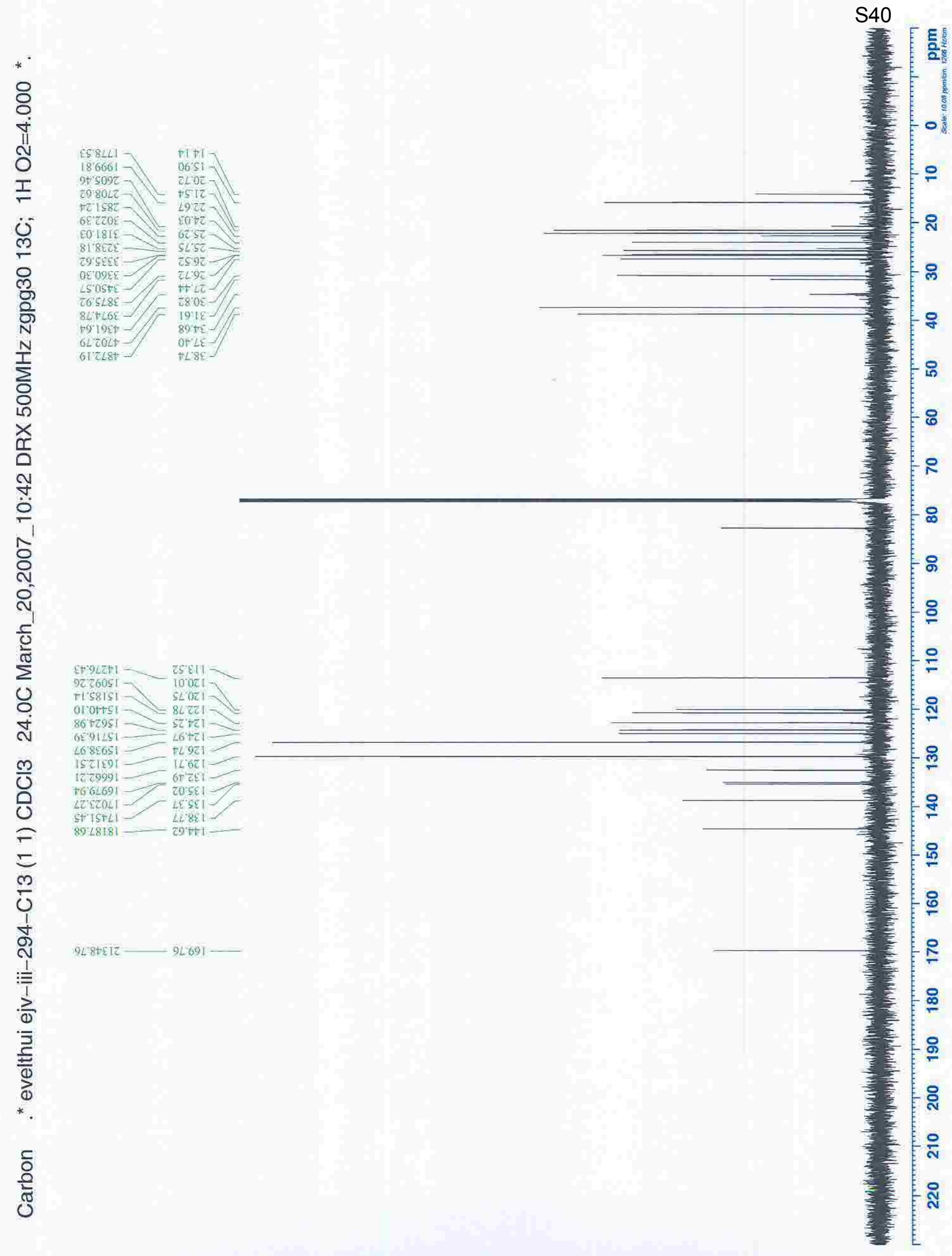




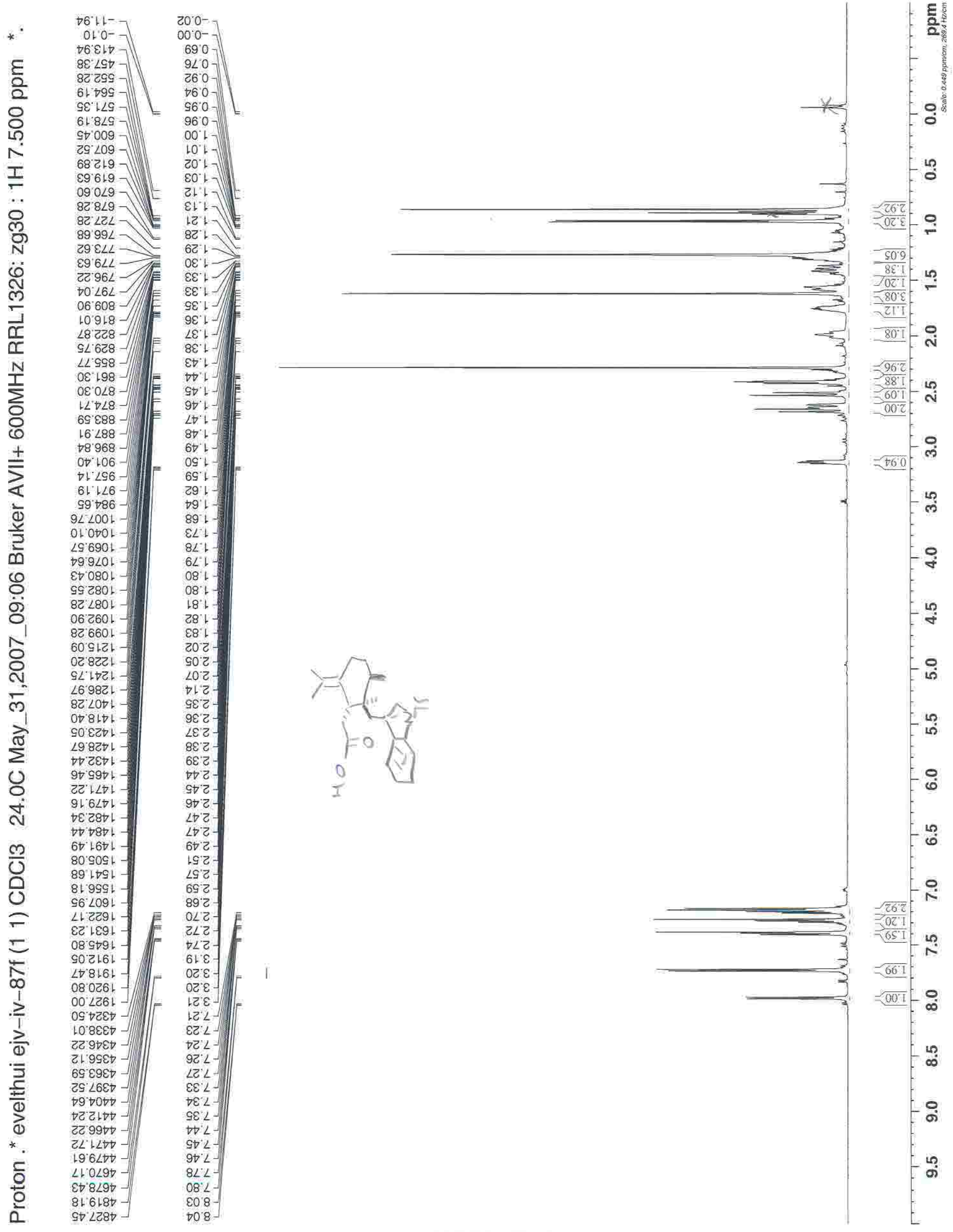




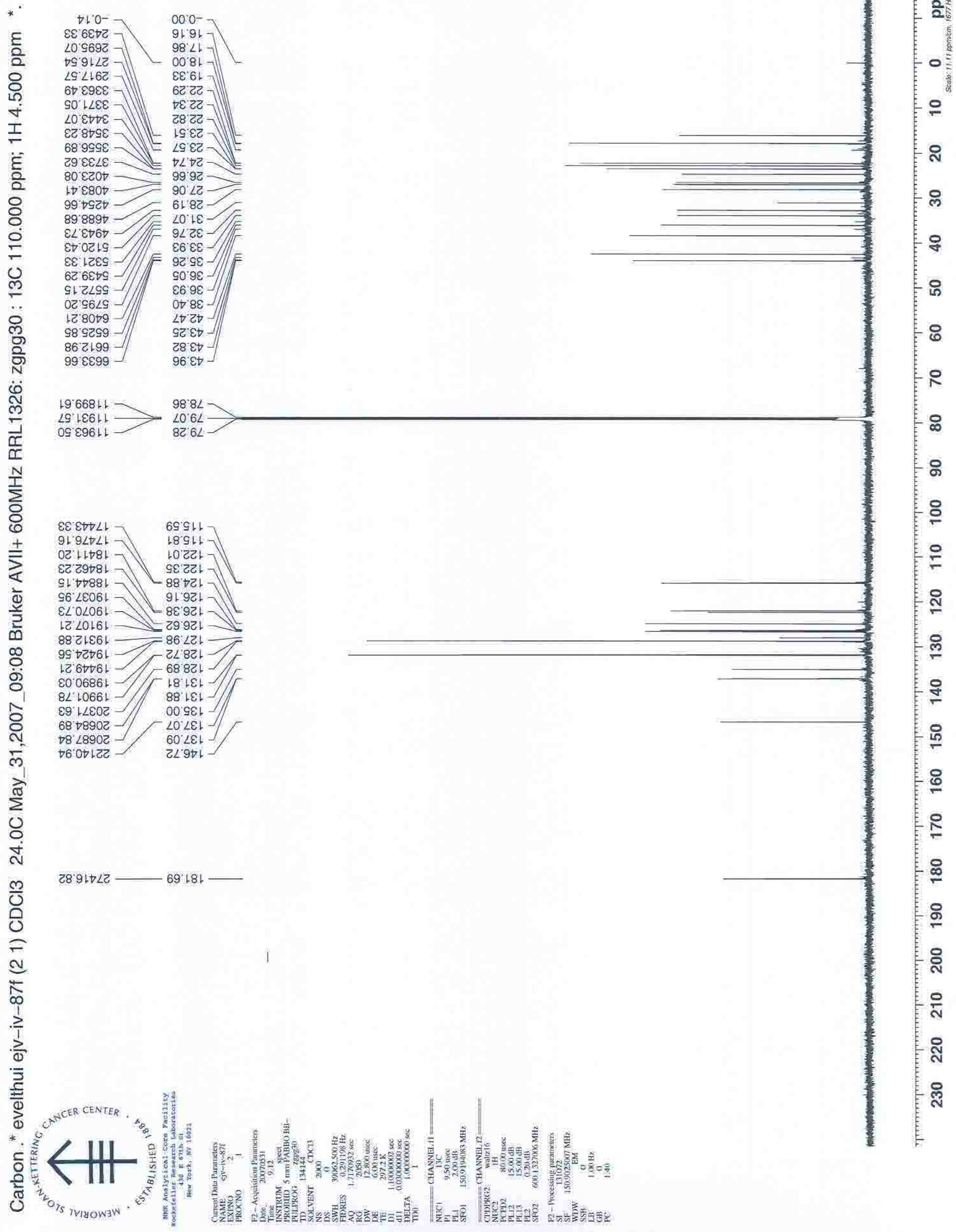




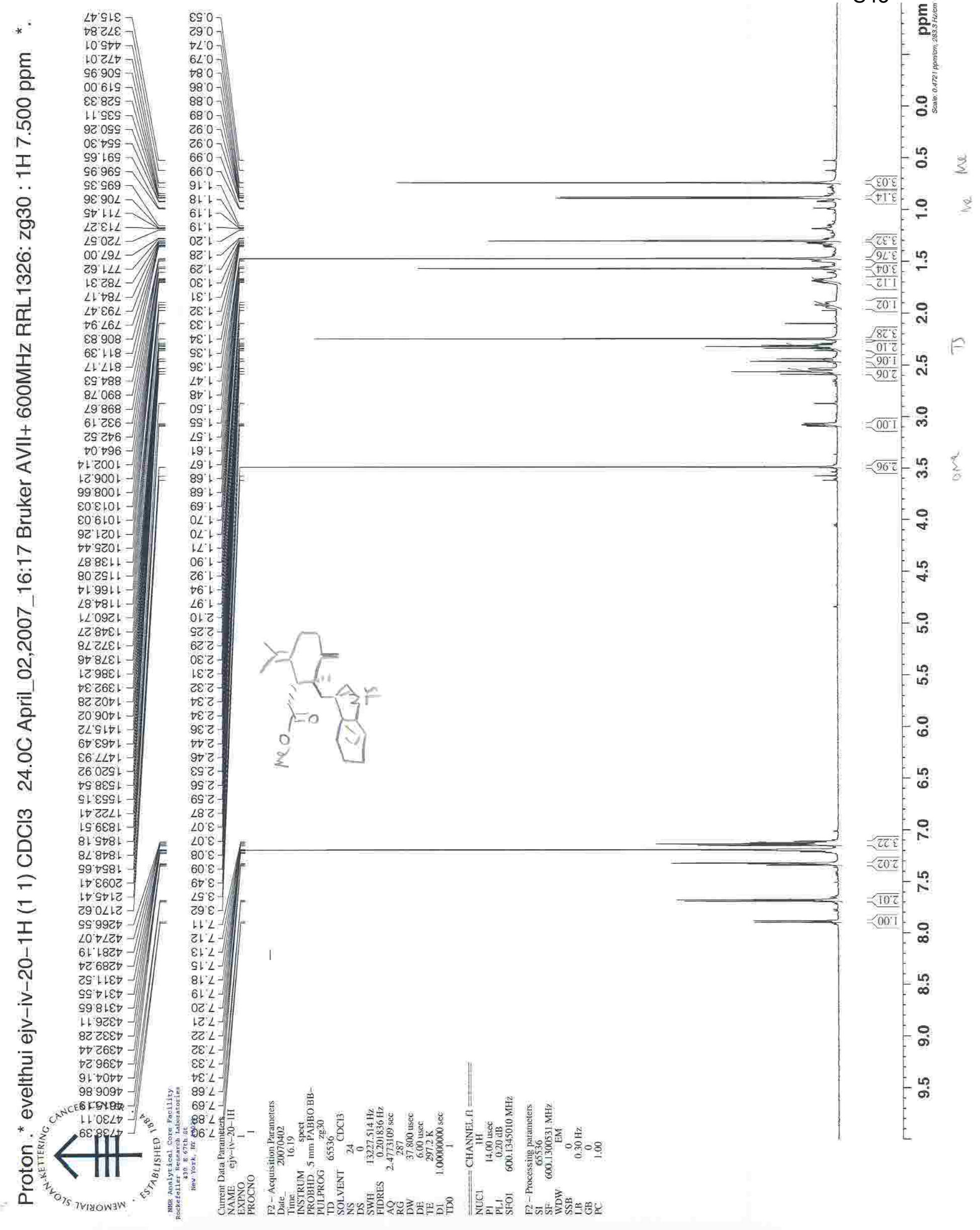




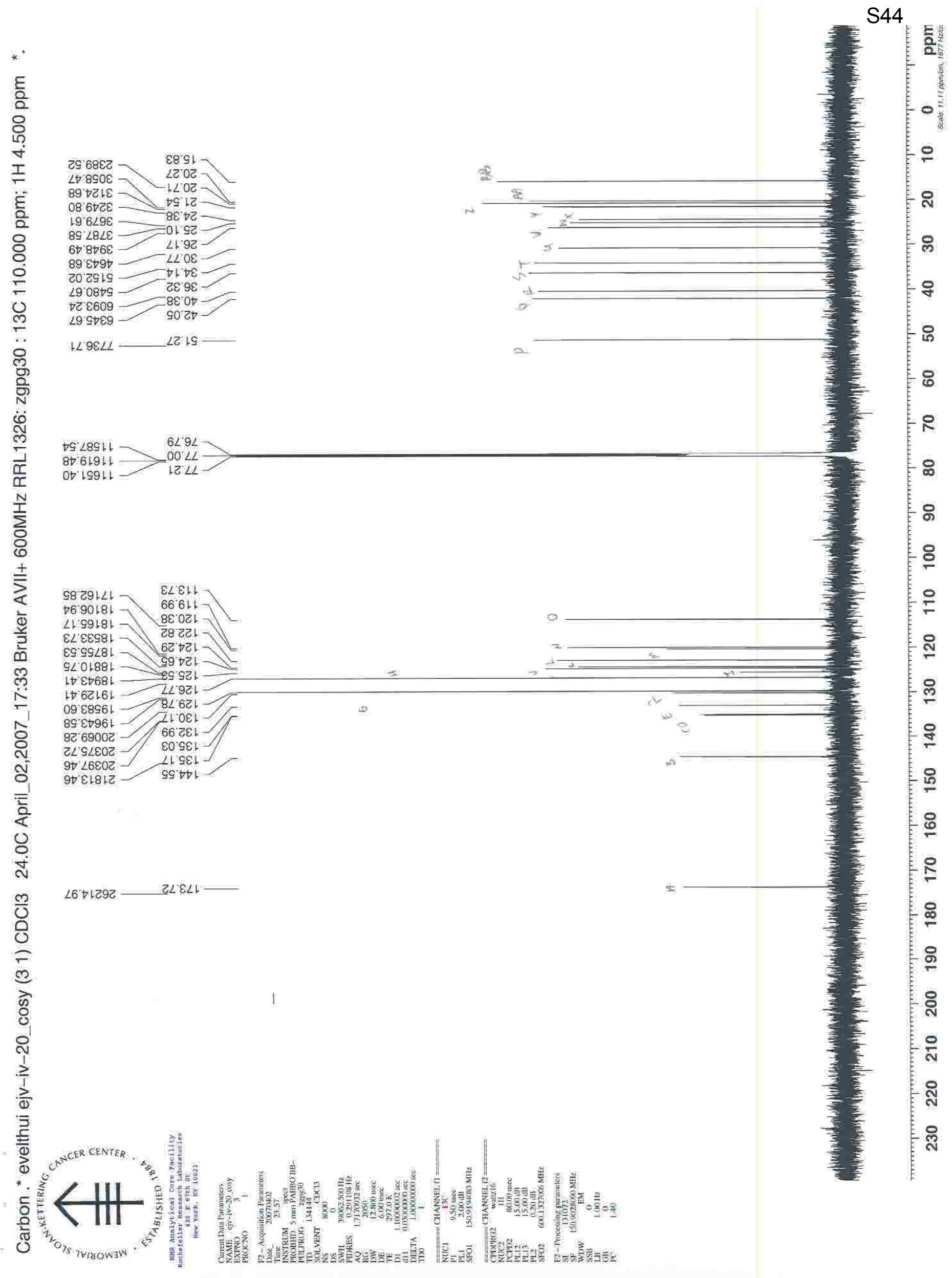




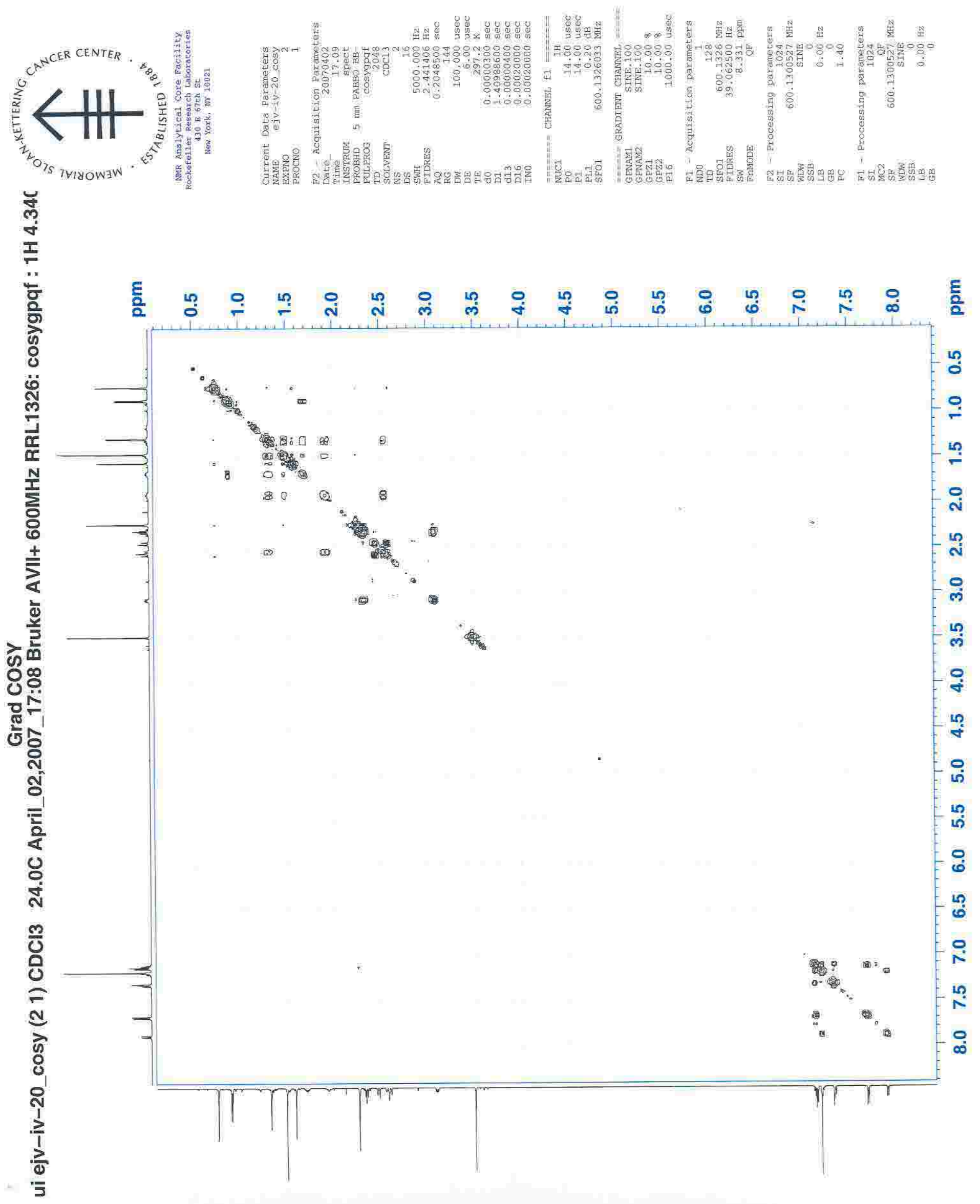


S46

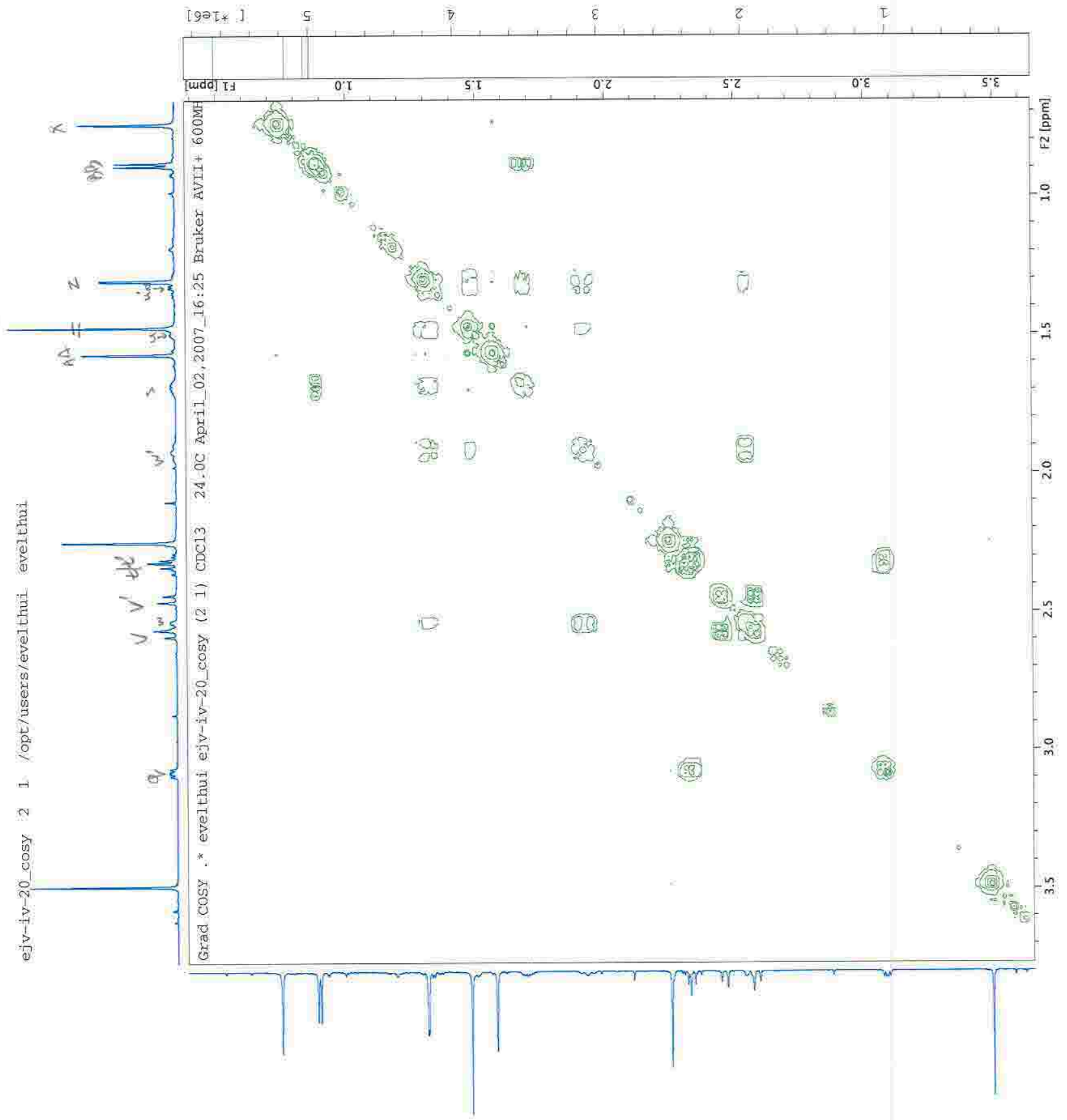




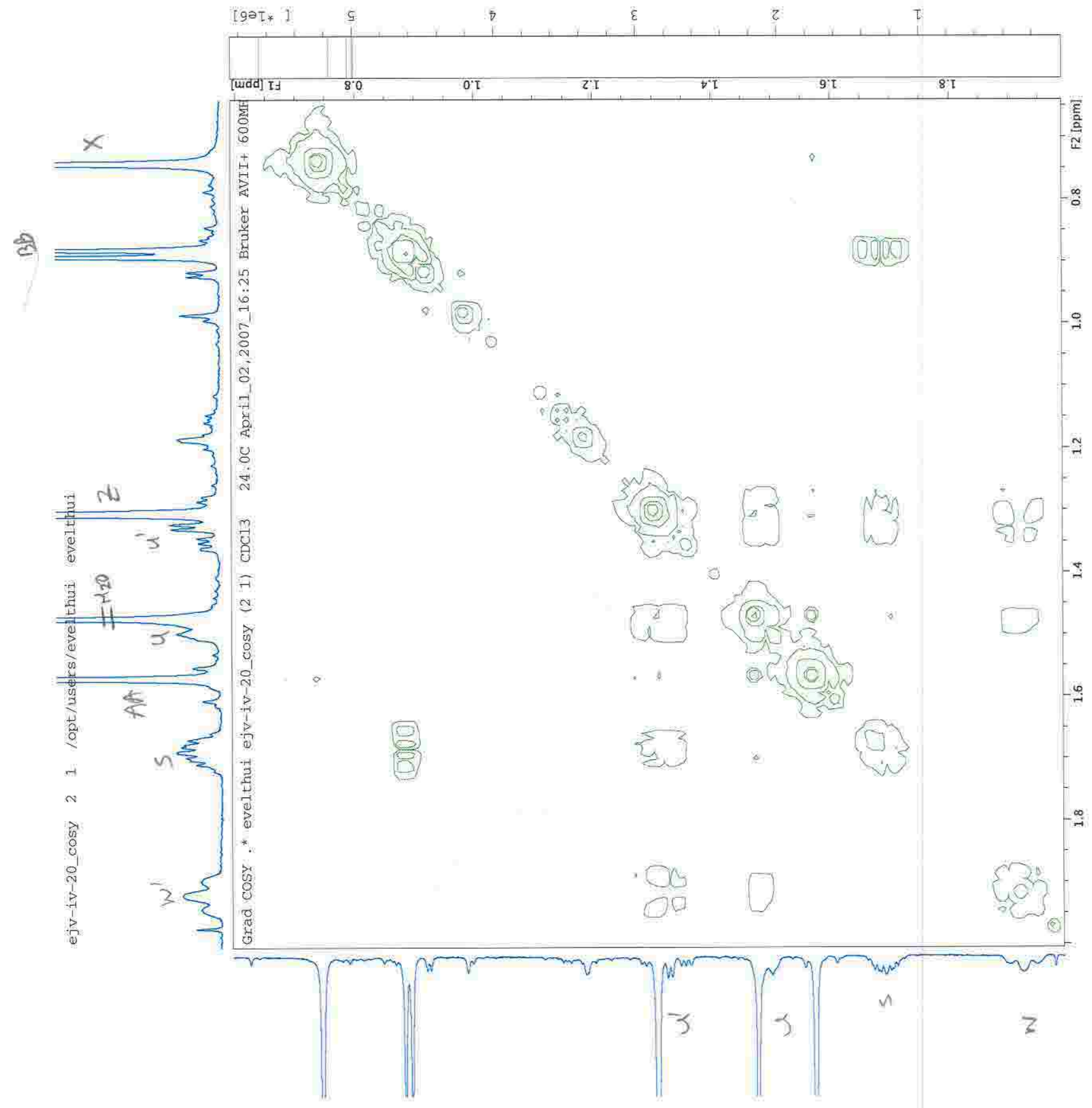




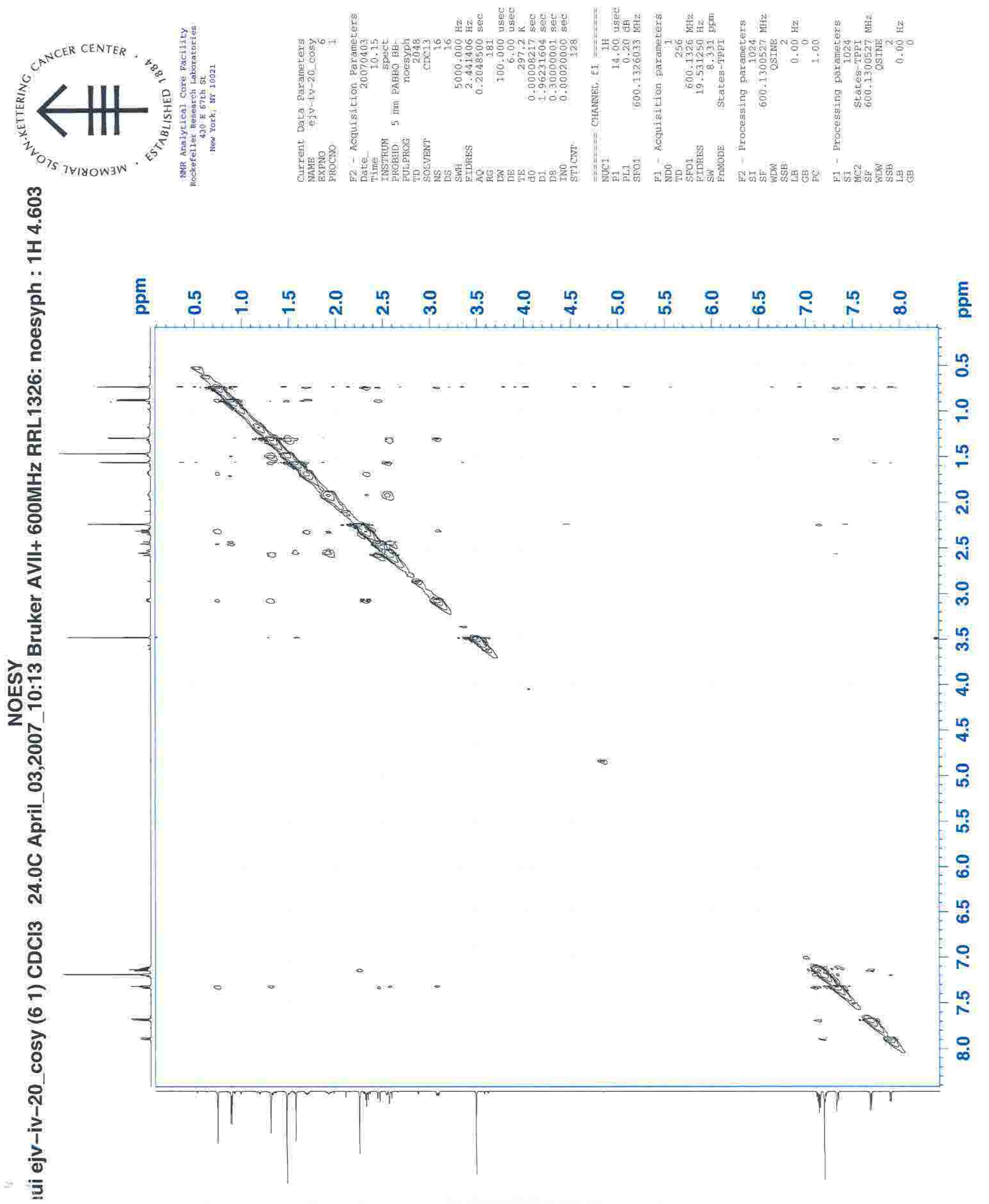




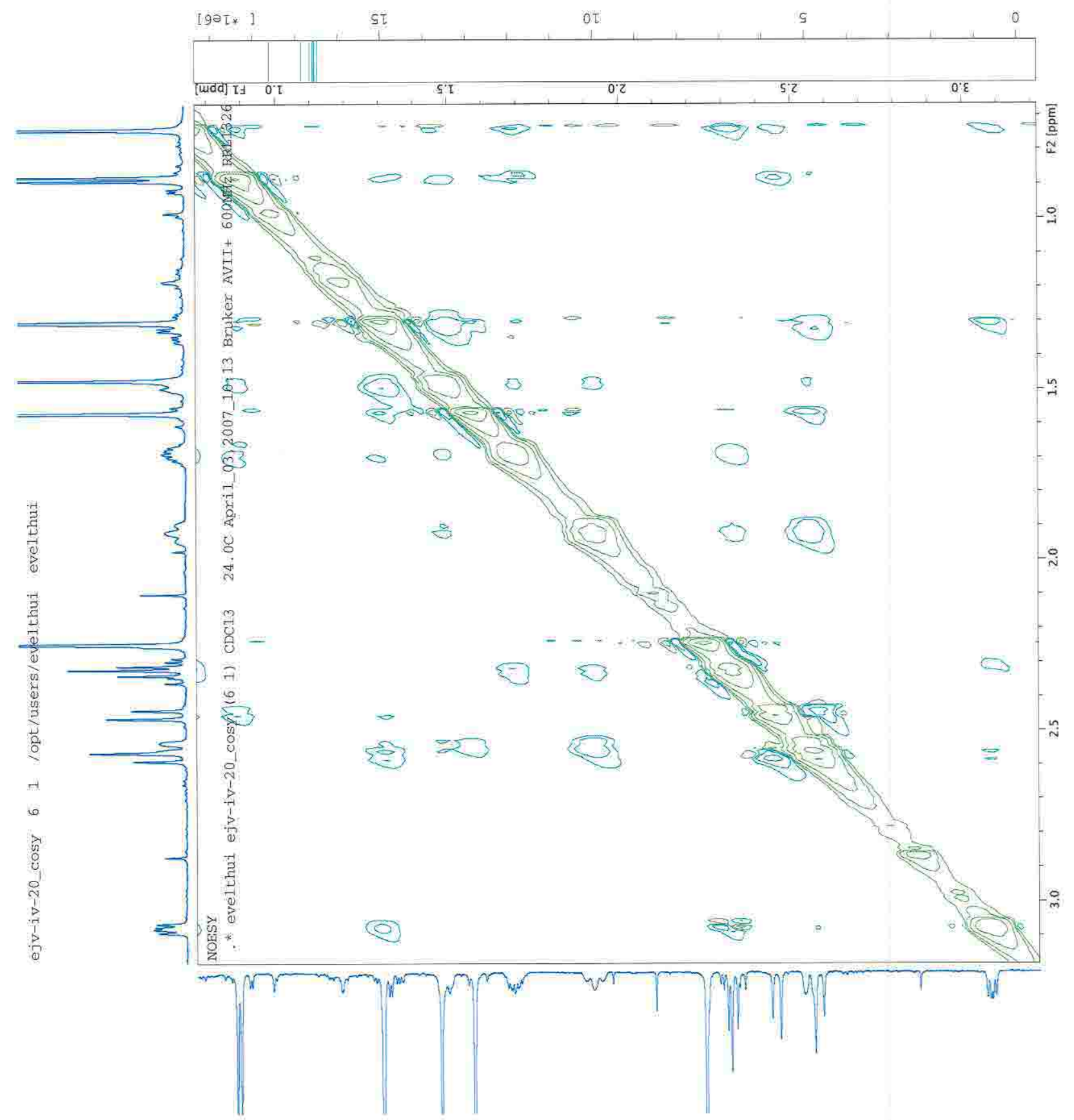




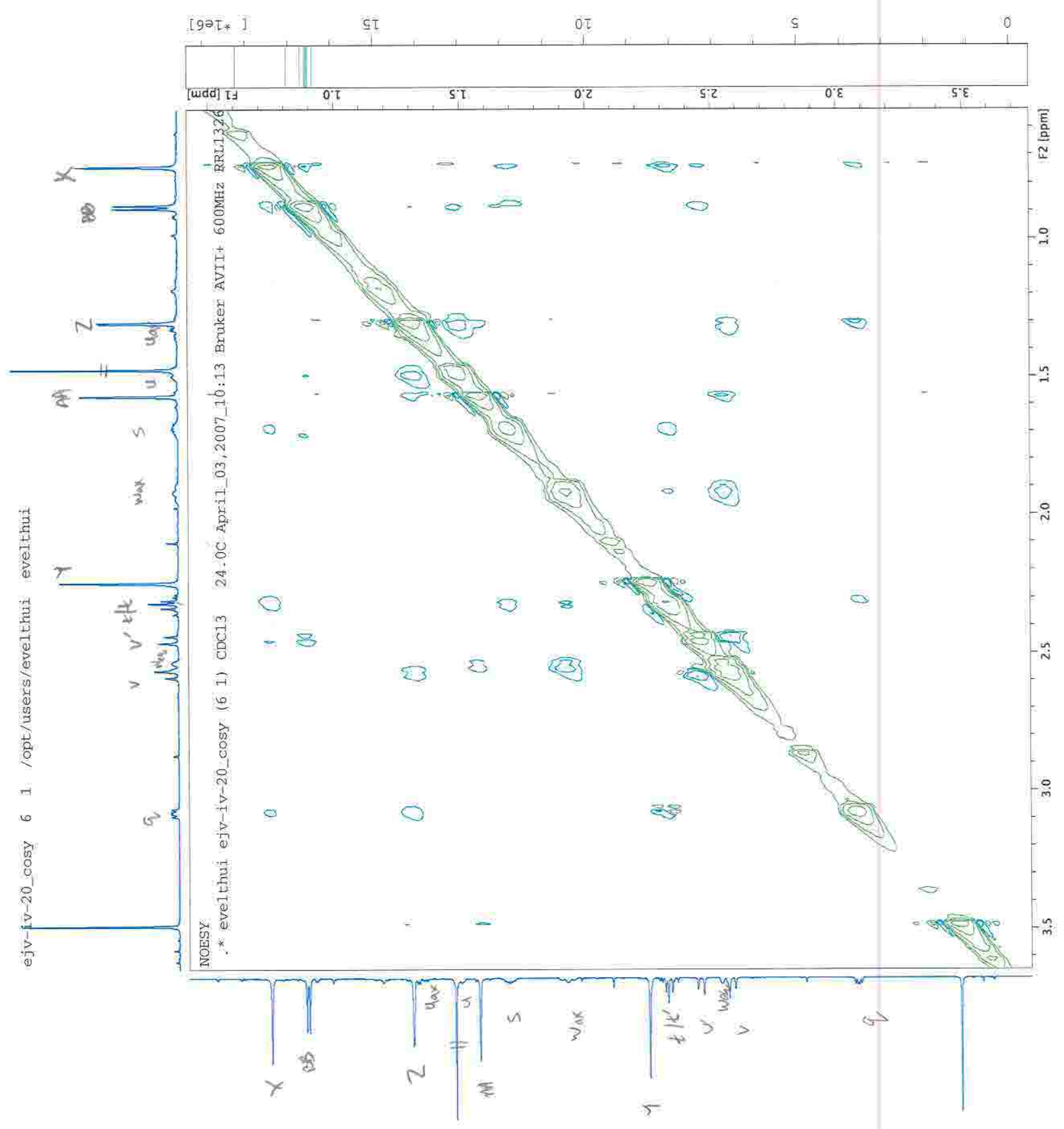




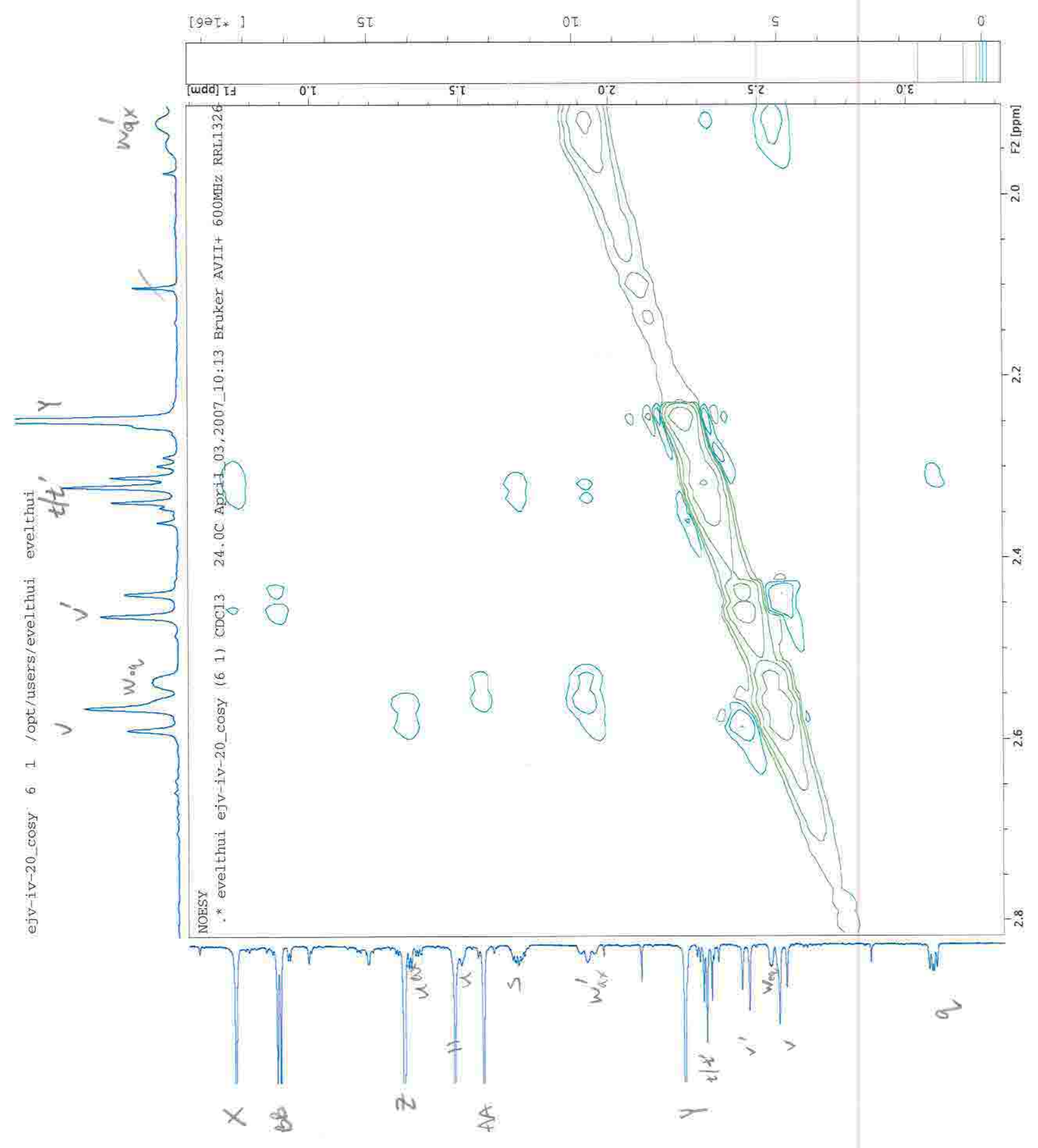




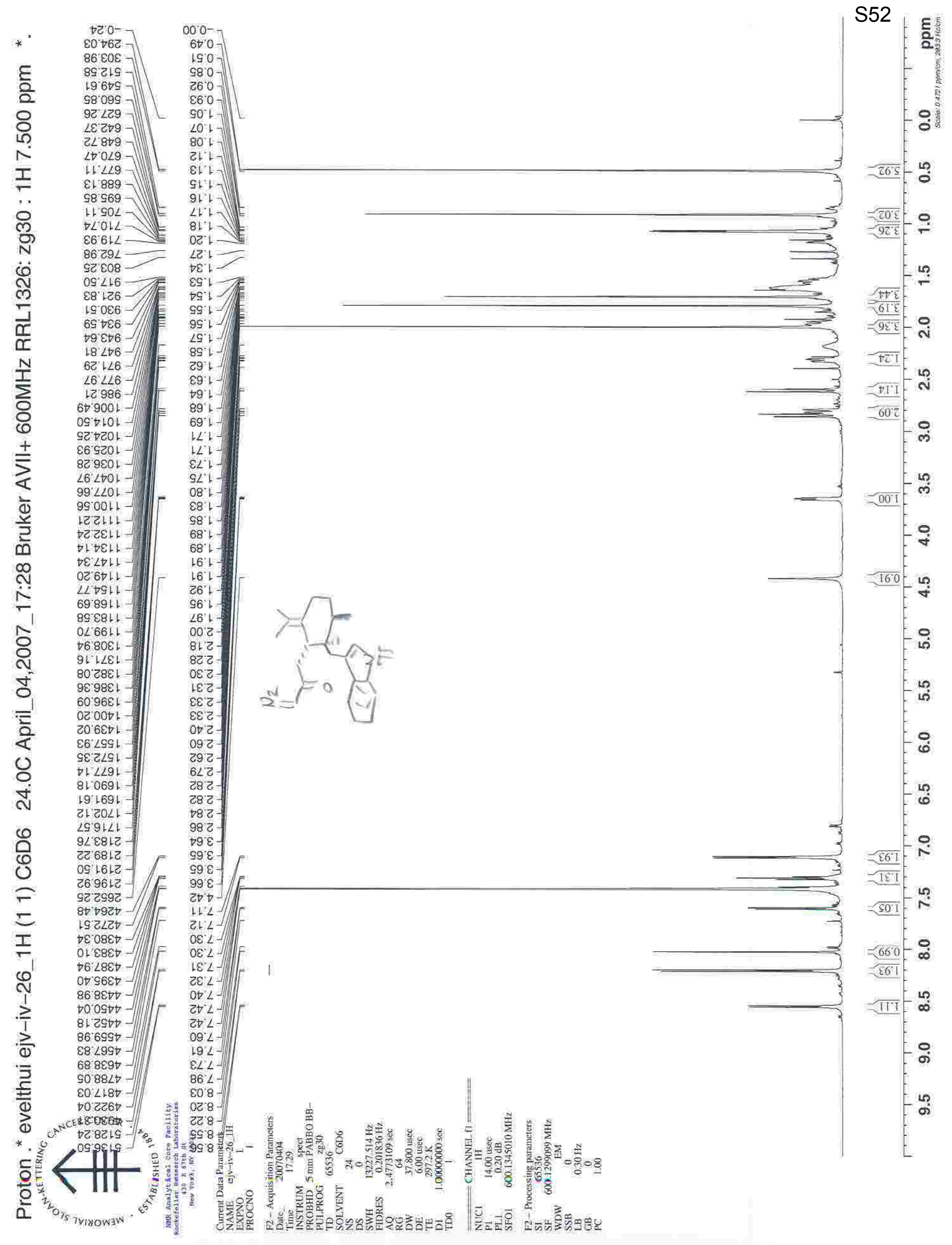




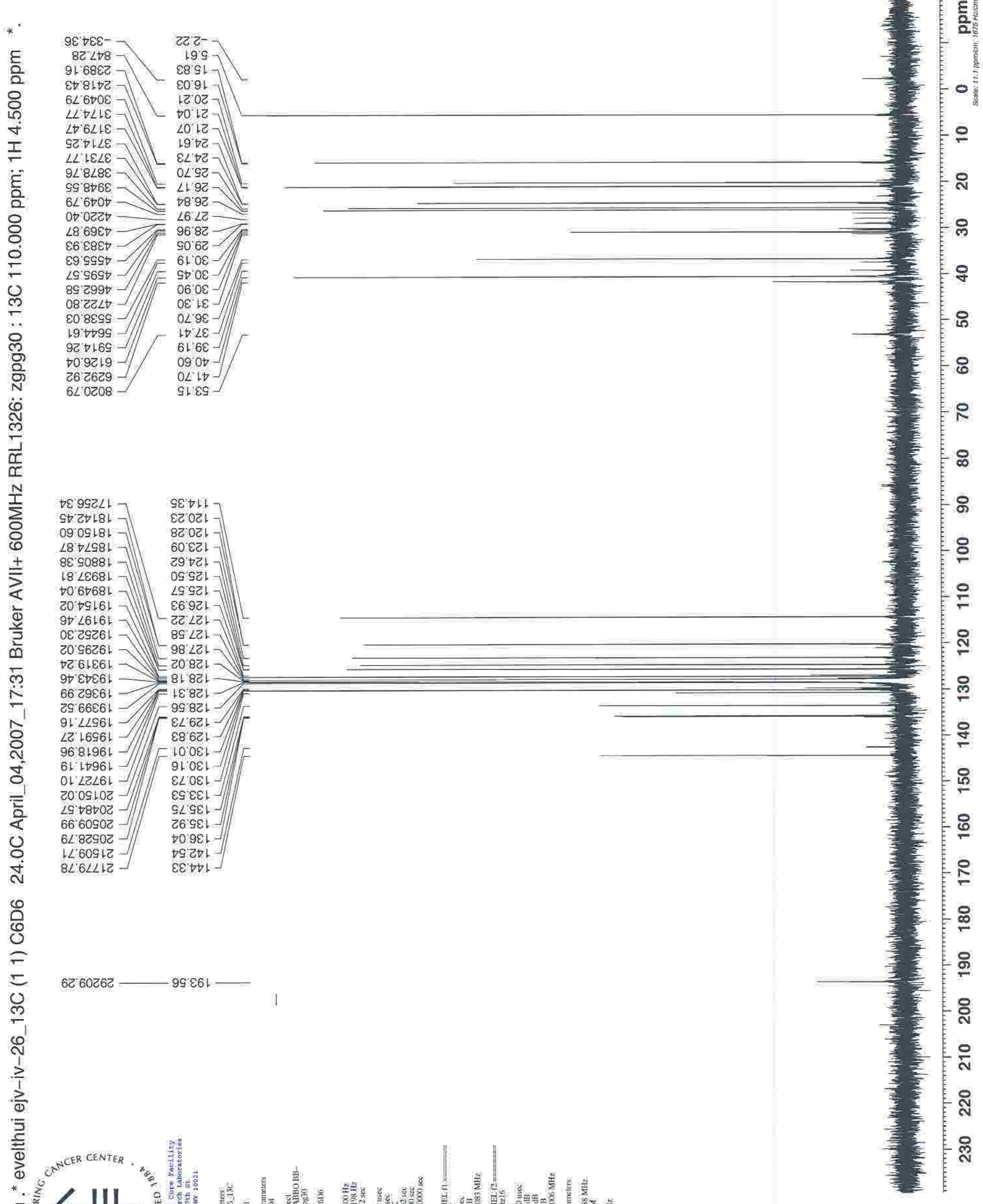




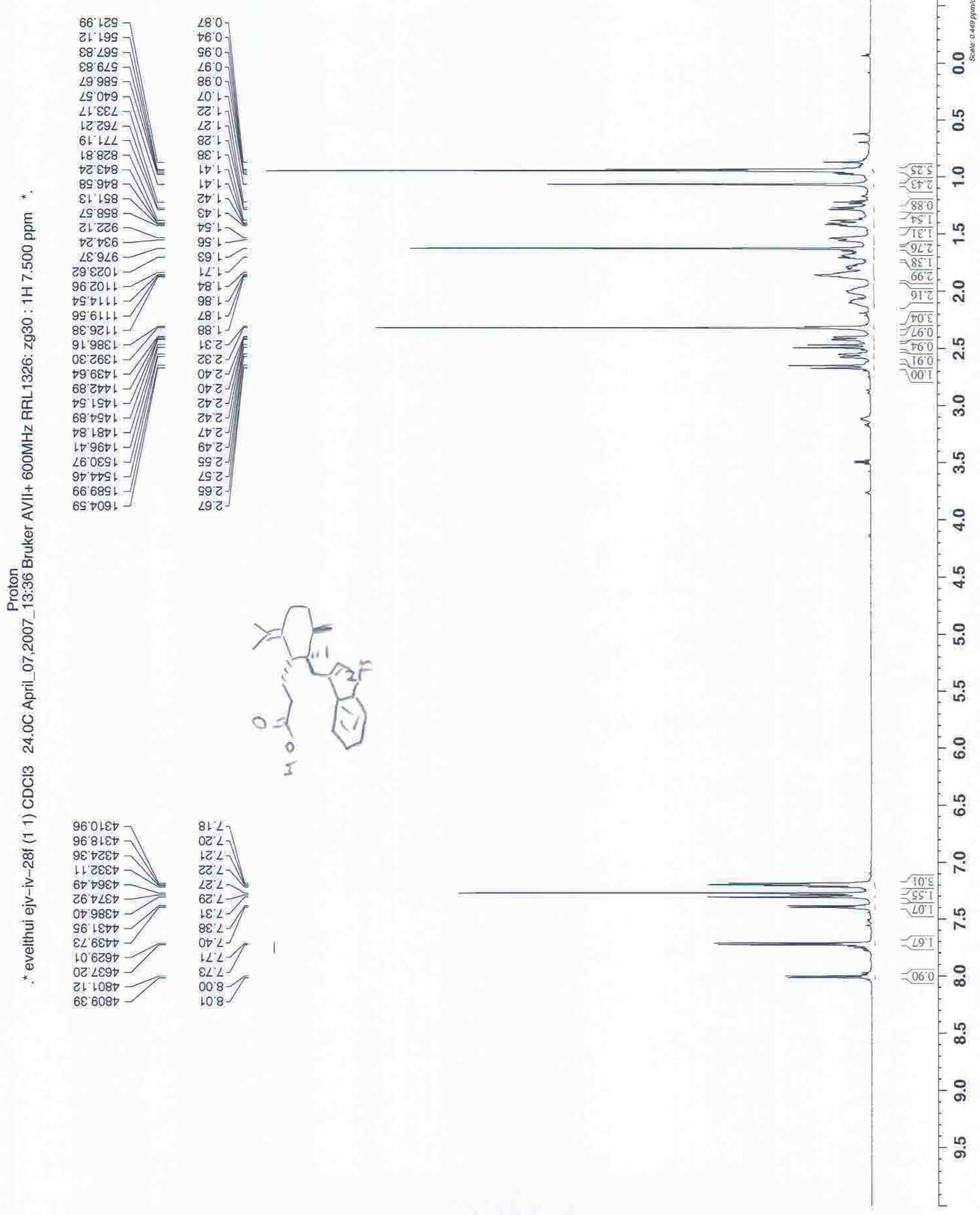




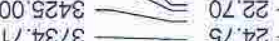

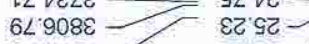

$8+\angle 100-29.92$

Zट $2995 \square 060 \varepsilon-$

0. $\varepsilon 28 t-96.1 \varepsilon$

t9.89t9 $\angle 1.98$

OL. EटL $~ 89^{\circ} 0 t$

Eเ' 9799

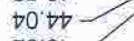

$98: \varepsilon+69$

$28 \mathrm{st}$

$898891-$

2902911

$089 L$

६९ ZS9L.

$2 Z \angle L$
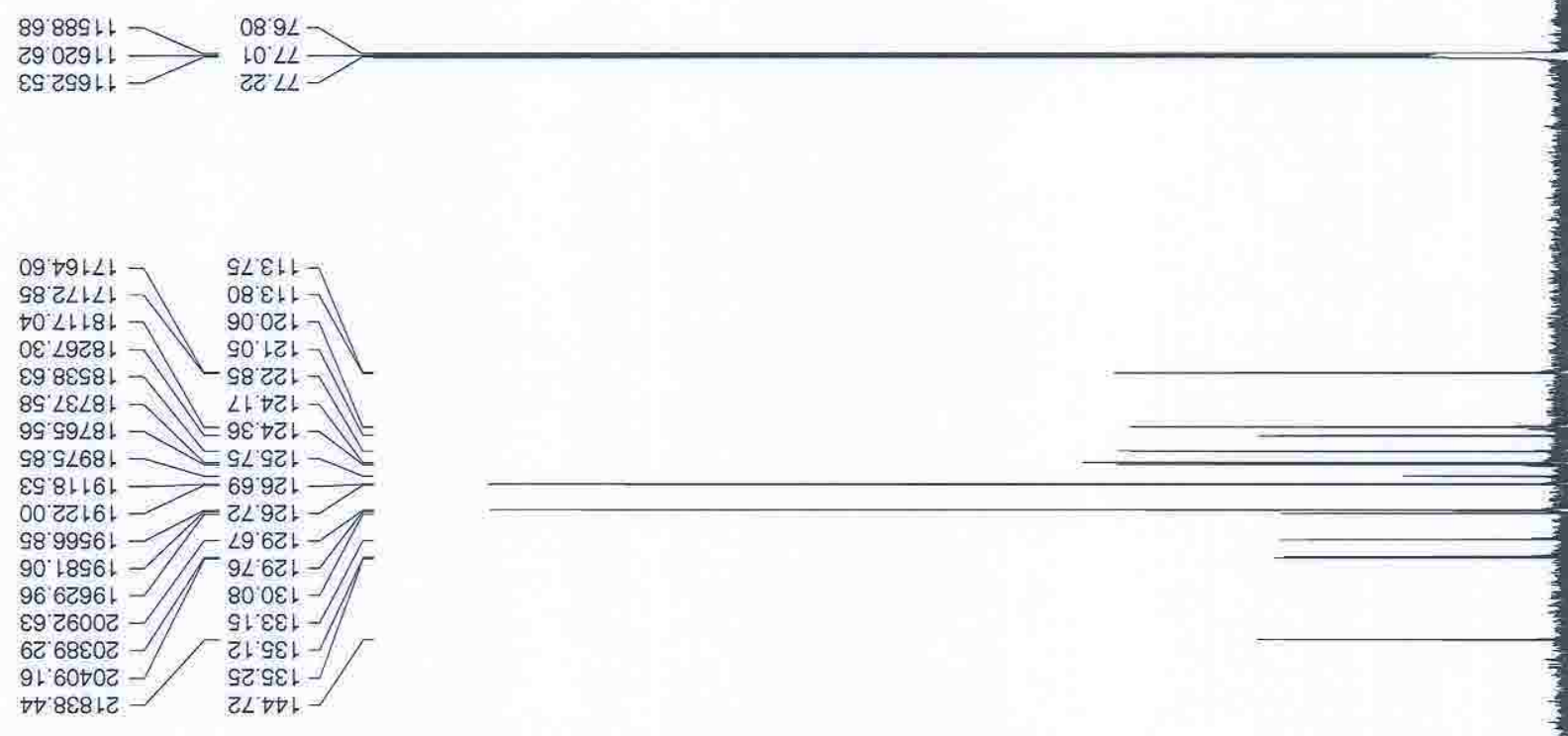

$\sum_{0}^{N}$

(1)

흔

0

ণ

$\frac{m}{0}$
0
0

V

ֻับ

政
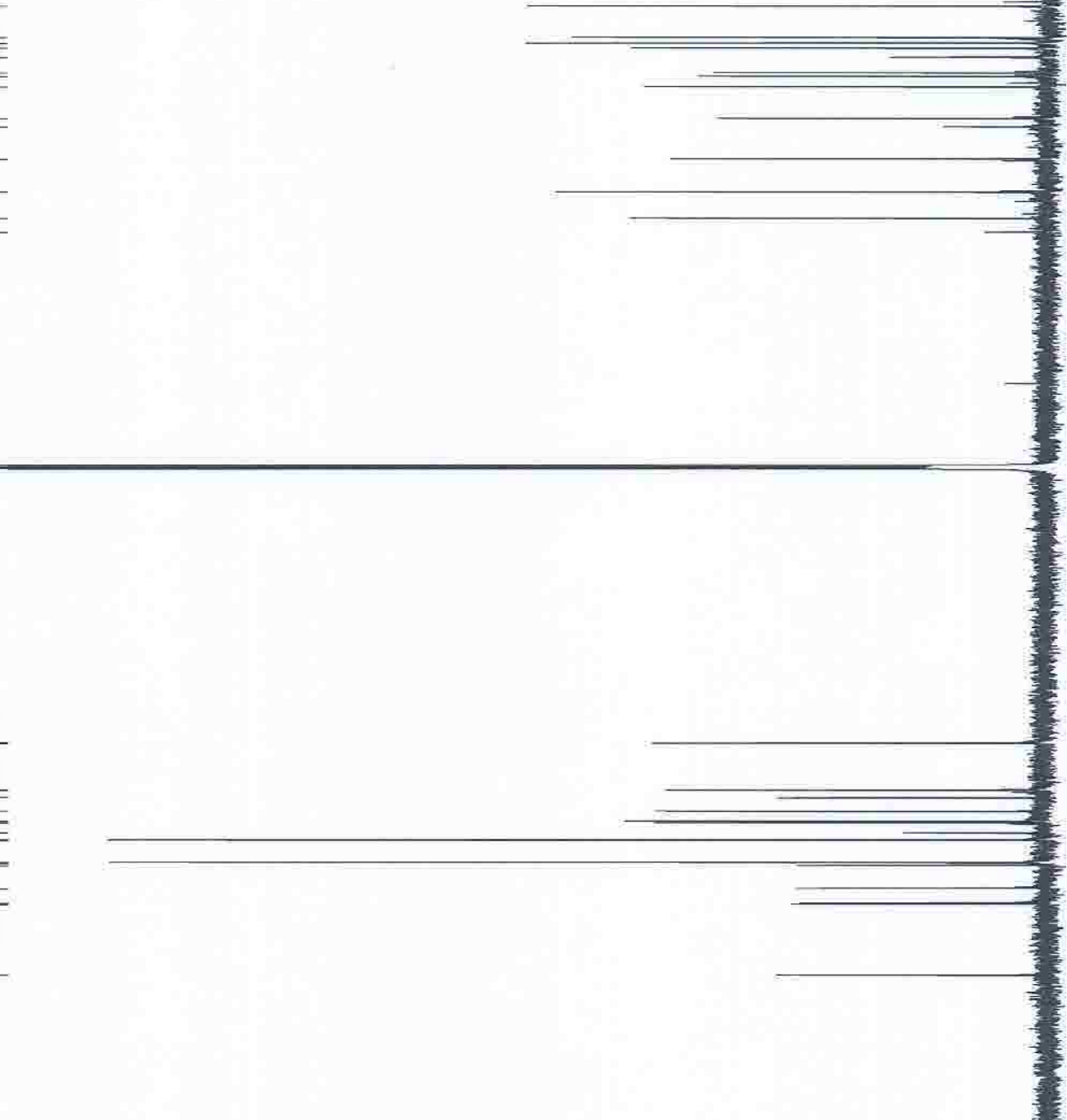
S56

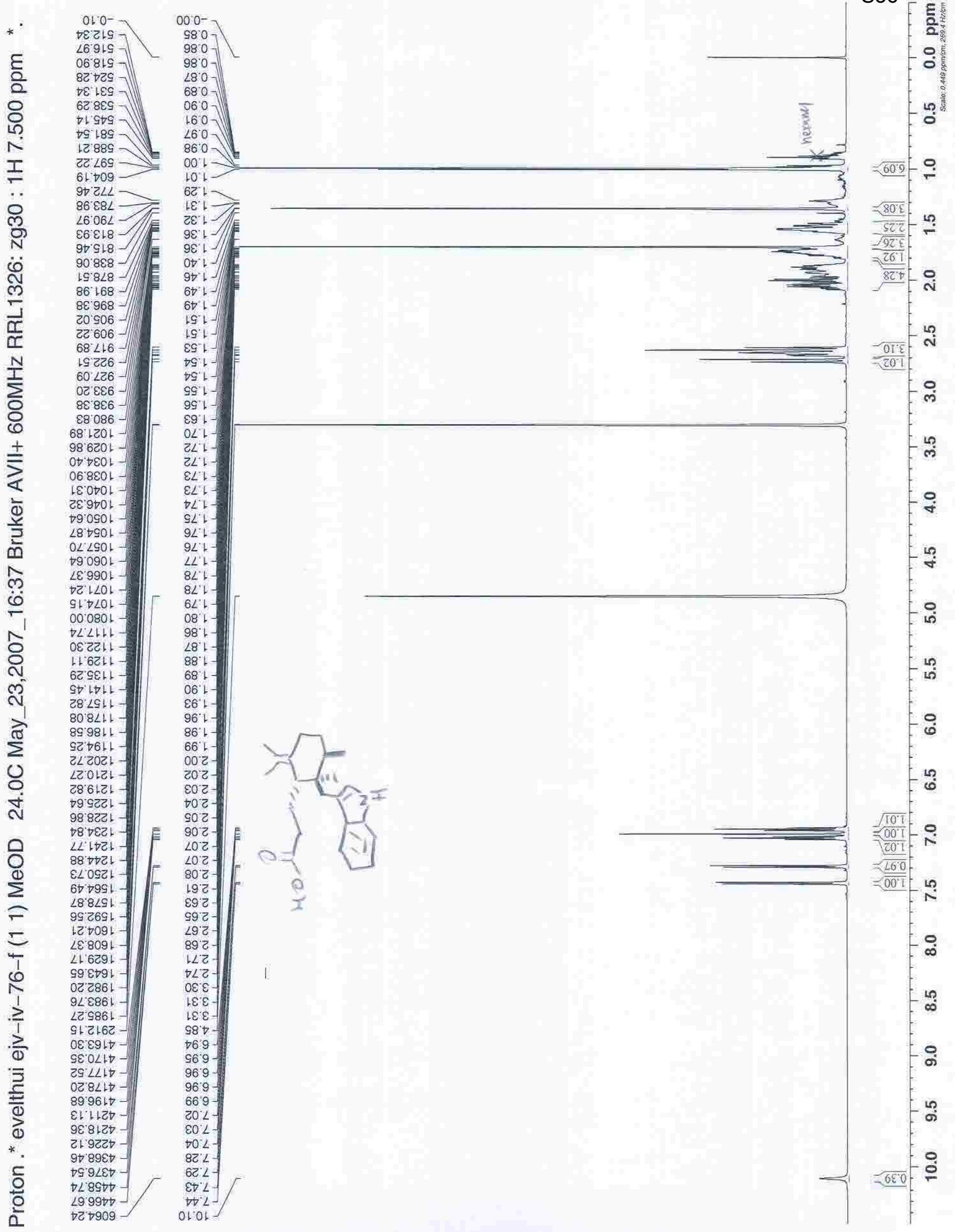


920812 $89^{\circ} \forall \angle D Z$

$88 \angle 90 \varepsilon$

$\nabla ! \nabla \angle L E$

$\angle 80 \angle Z E$

$126 \angle 98$

$88^{\circ} 129 \varepsilon$

$\checkmark \nabla 606 \varepsilon$

$\checkmark L \angle E 6 \varepsilon$

$20 \angle 968$

$99602 t$

$0<9 \angle 87$

$\nabla L \angle 667$

$\nabla \varepsilon: 28 \varepsilon 9$

69'9699

$9 \varepsilon \div 1 \varepsilon 9$

1LLL89

ธट ยะ\&L

$\angle 9 \rightarrow 9 E L$

$80.9 \angle \varepsilon L$

$6 \forall \angle 6 \& L$

$16.8 \mathrm{~L} t \mathrm{~L}-$

$\varepsilon \mathcal{E} O \Delta \backslash L$

$9<+9 \gg<-$

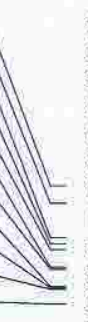

$9 t \nabla 1-$
Ot 91

$\varepsilon \varepsilon \cdot 0 z$

89.12

ZL $\varepsilon 2$

$00 \nabla 2$

6092

टZ 92

$06^{\circ} \angle Z$

टह टह -

टL टब

OL 98

$9 L \angle E-$

98 it

$89^{\prime} 9 t$

$098 \mathrm{t}$

$\nabla \angle 8 \nabla-1$

$88.8 t-$

20.67

9.6 $6 \mathrm{D}$

เE. 60

st $6 b]$

8788891

09.9689 !

$88^{2} \mathrm{Z} 60 \mathrm{LL}$

$69^{\prime}\llcorner 86 \angle h$

$99 \angle 2 L 81 \longrightarrow \varepsilon 1021 \longrightarrow$

$9108 \varepsilon 8$ - — 08121

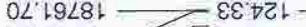

$8998 L 8 h-6 \nabla \nabla 2 L$

21 $6706 \mathrm{~L}-\varepsilon 292 \mathrm{~L}$

乙† $9 \varepsilon \angle 61>8 \angle 0 \varepsilon L$

$972 L 661-9 \varepsilon Z \varepsilon$ L

$\checkmark 96 \angle L O Z=O L L E L$

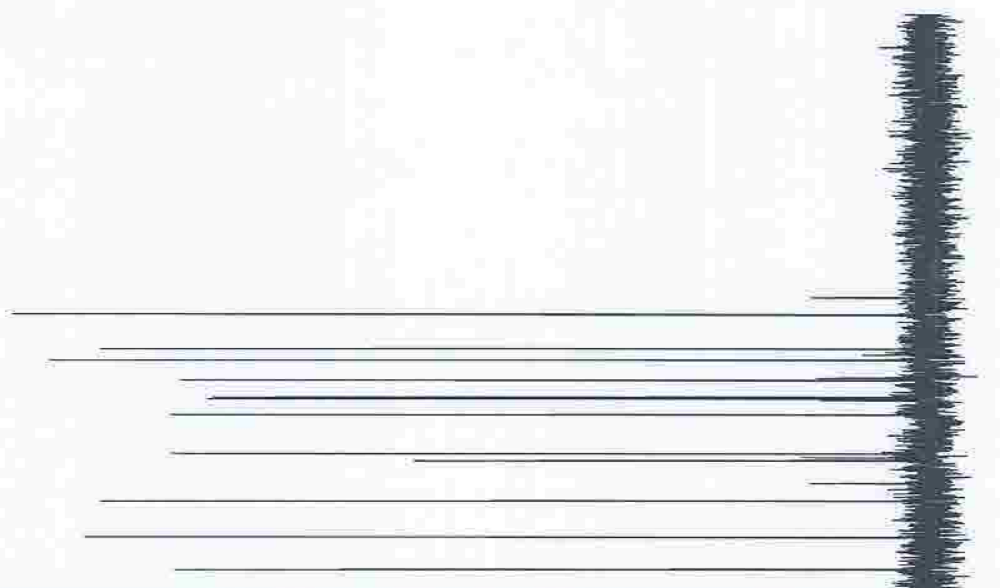

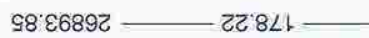




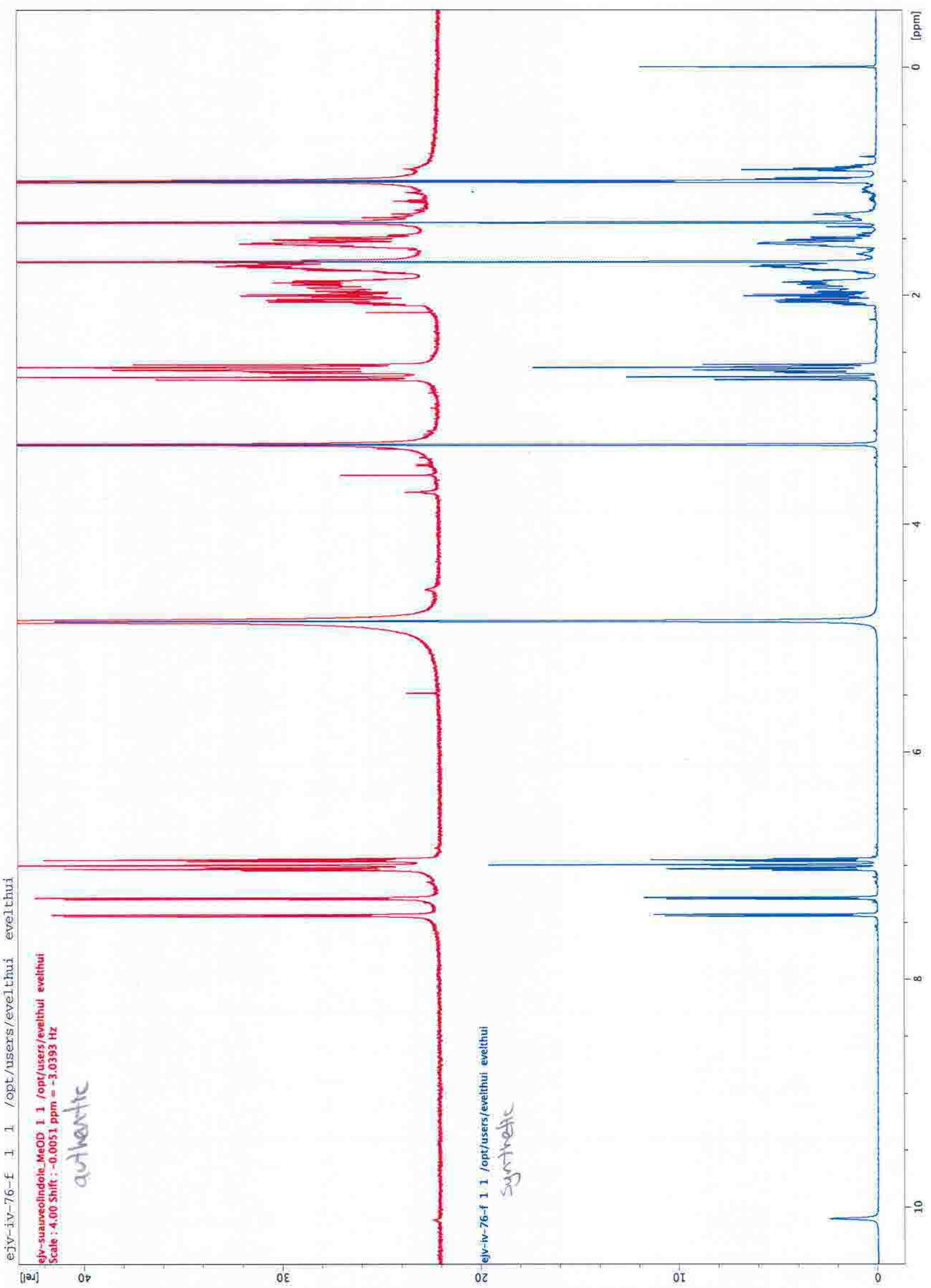


S59

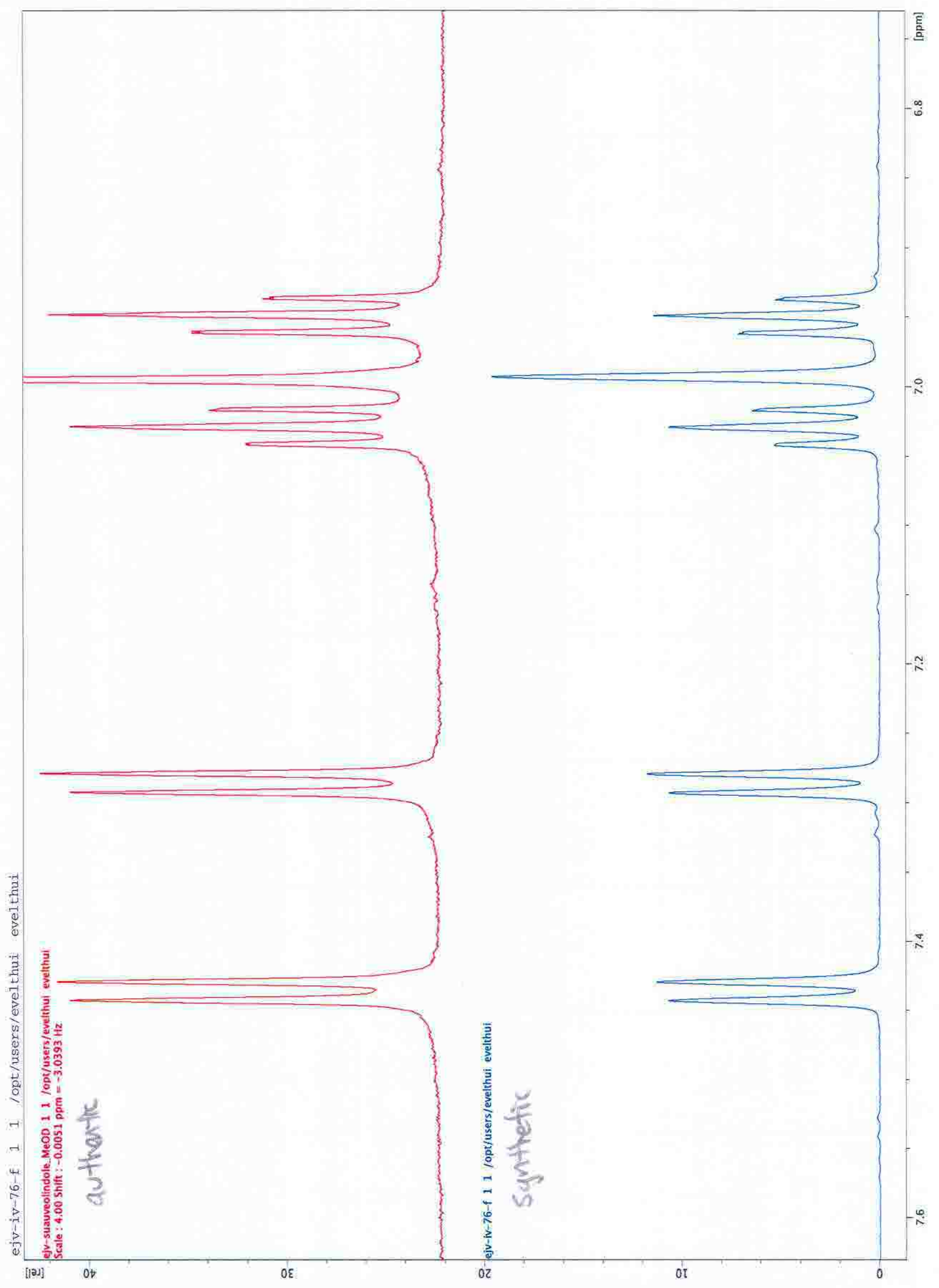


S60

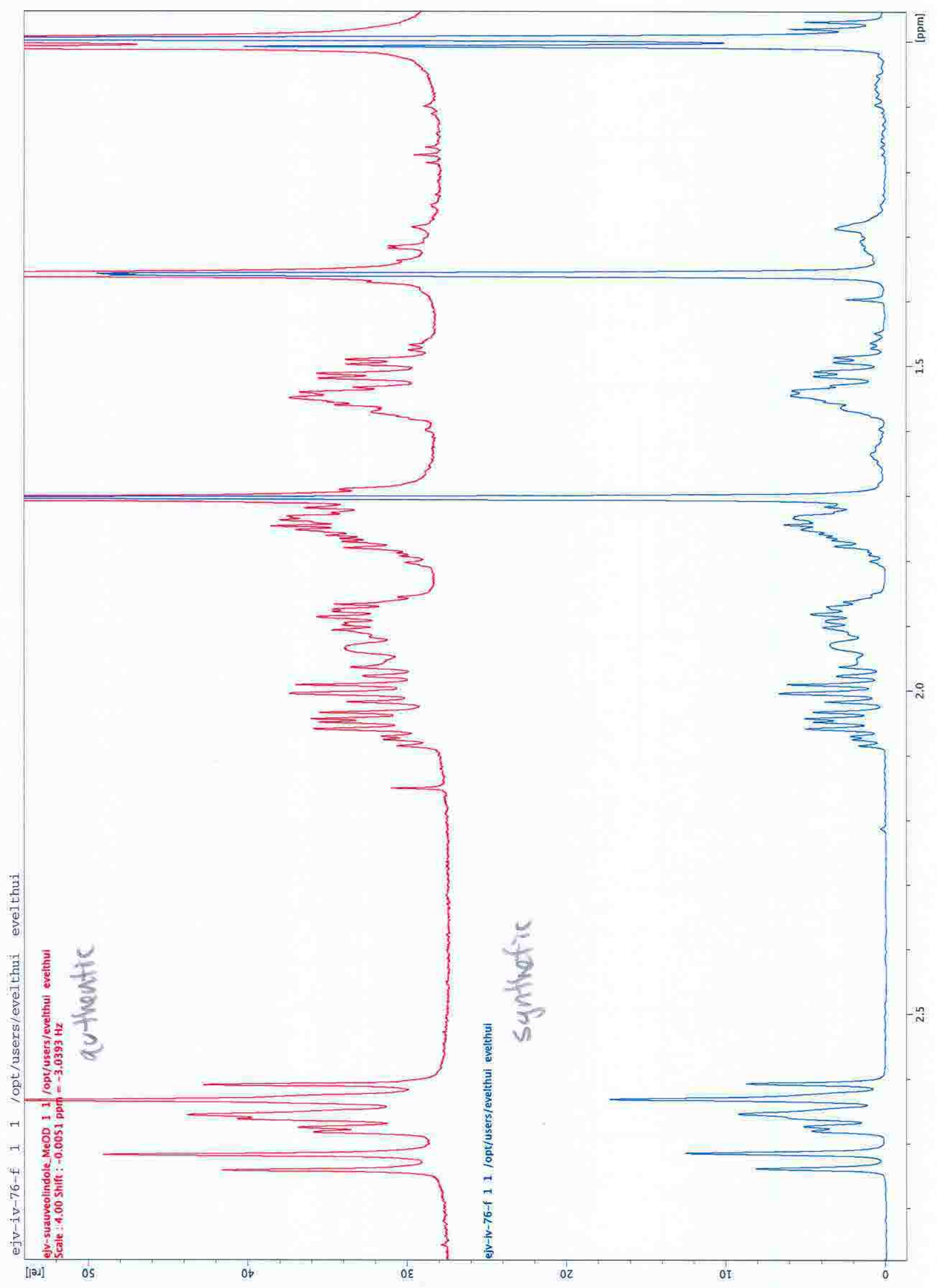




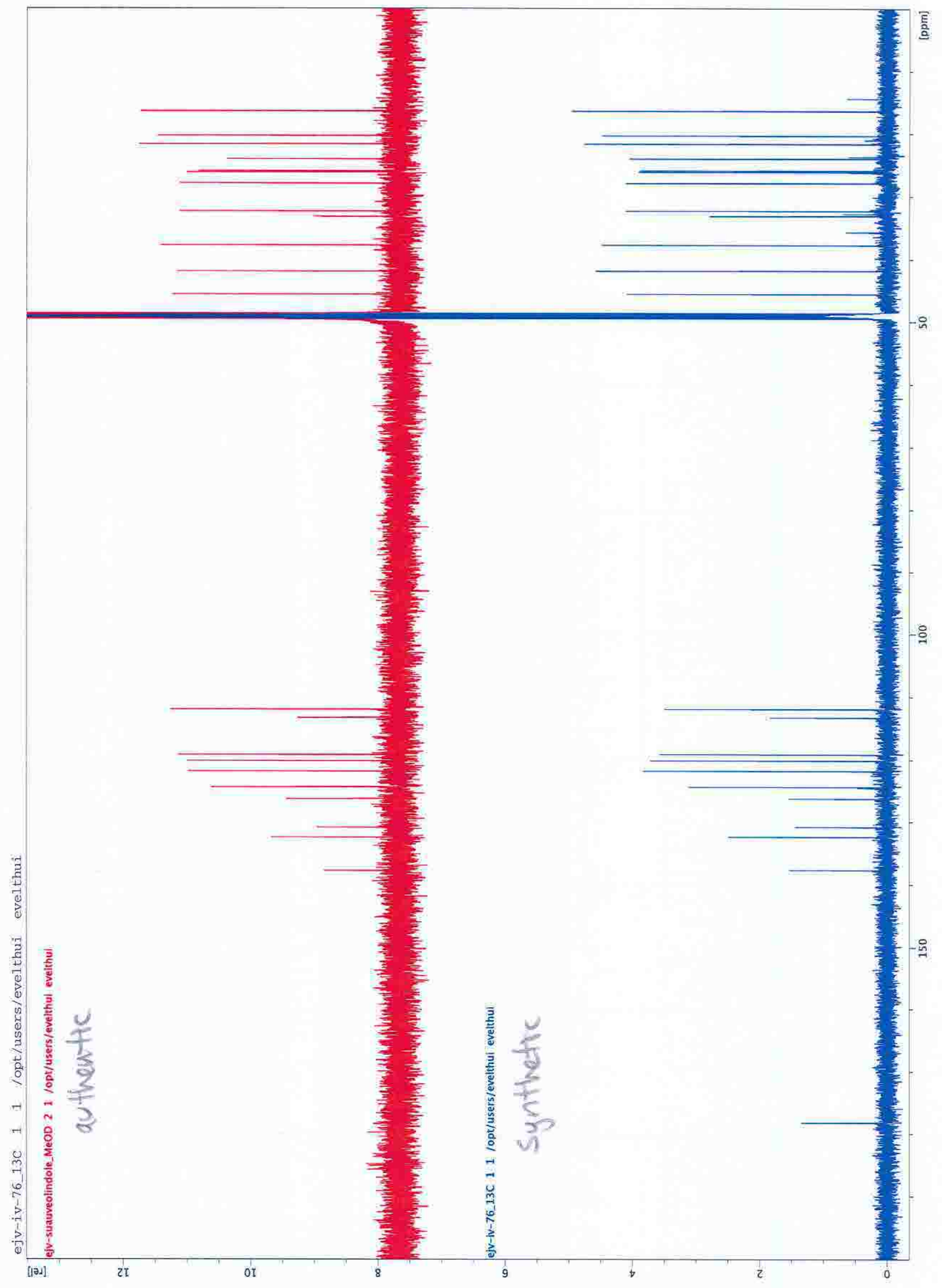




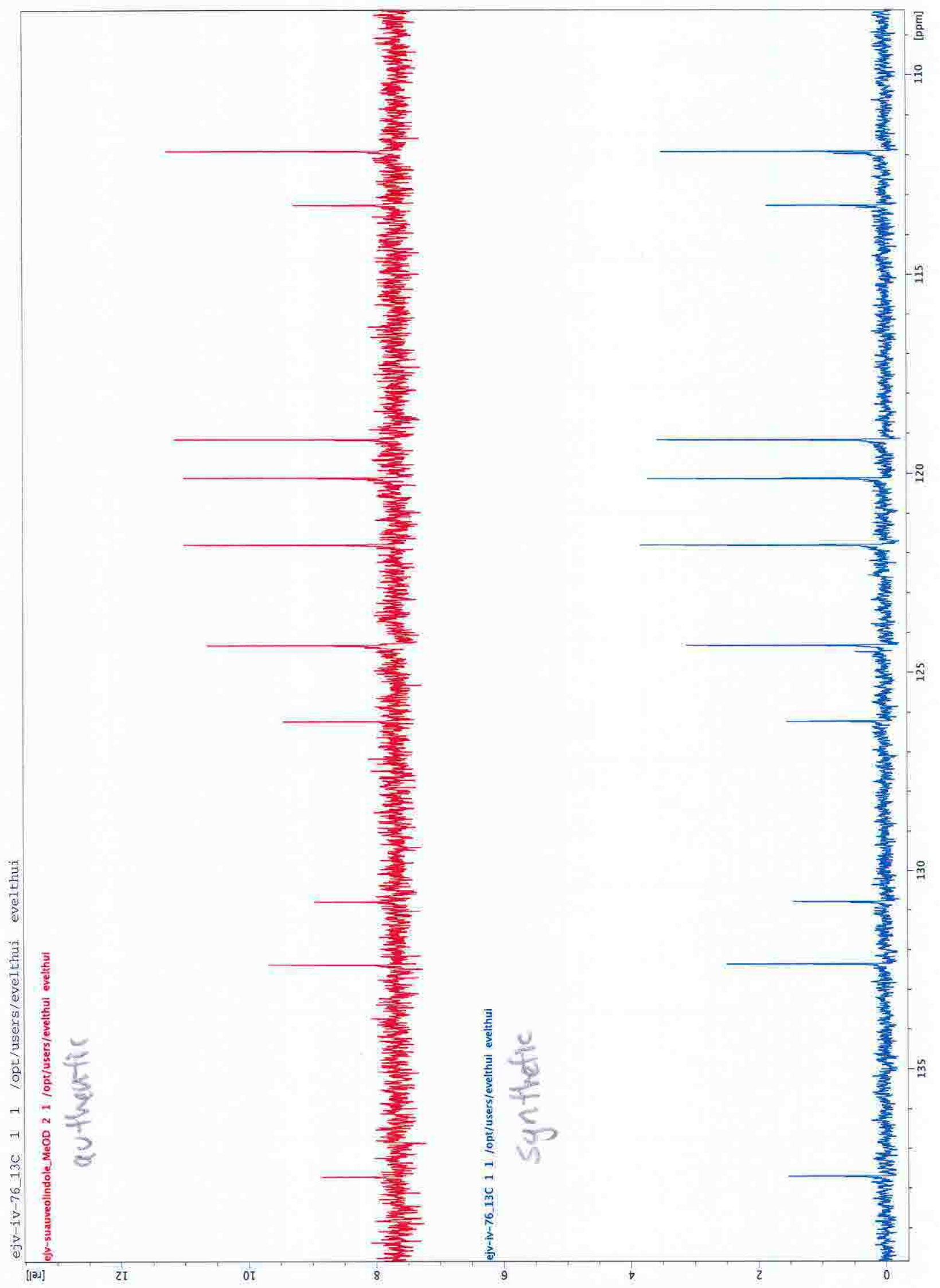




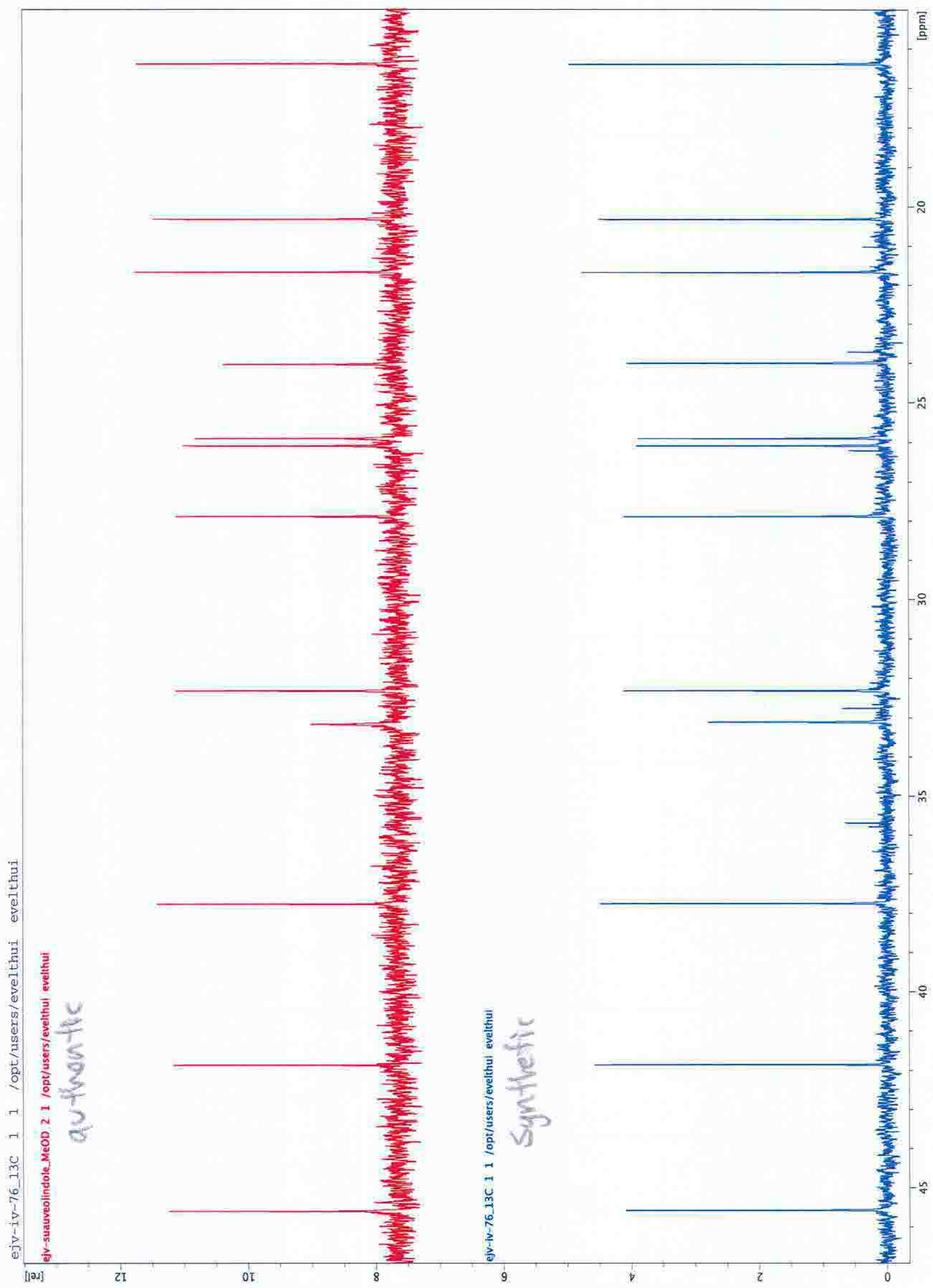

